2015-02-06

\title{
Sketch-Based Dance Choreography
}

\author{
Moghaddam, Elahe
}

Moghaddam, E. (2015). Sketch-Based Dance Choreography (Unpublished master's thesis).

University of Calgary, Calgary, AB. doi:10.11575/PRISM/27796

http://hdl.handle.net/11023/2092

Downloaded from PRISM Repository, University of Calgary 


\title{
THE UNIVERSITY OF CALGARY
}

\author{
Sketch-Based Dance Choreography
}

by

Elahe Rajabzadeh Moghaddam

\author{
A THESIS \\ SUBMITTED TO THE FACULTY OF GRADUATE STUDIES \\ IN PARTIAL FULFILLMENT OF THE REQUIREMENTS FOR THE \\ DEGREE OF MASTER OF SCIENCE
}

DEPARTMENT OF COMPUTATIONAL MEDIA DESIGN

CALGARY, ALBERTA

December, 2014

(c) Elahe Rajabzadeh Moghaddam 2014 


\section{THE UNIVERSITY OF CALGARY FACULTY OF GRADUATE STUDIES}

The undersigned certify that they have read, and recommend to the Faculty of Graduate Studies for acceptance, a thesis entitled "Sketch-Based Dance Choreography" submitted by Elahe Rajabzadeh Moghaddam in partial fulfillment of the requirements for the degree of MASTER OF SCIENCE.

Prof. Richard Levy

Faculty of Environmental Design

Prof. Faramarz Samavati

Department of Computer Science

Prof. Gerald Hushlak

Department of Art

Prof. Larry Katz

Faculty of Kinesiology

Date 


\begin{abstract}
Sketching and doodling are two techniques commonly used by choreographers to design a dance sequence. These sketches usually represent the trajectory of the dancer in the scene. A set of annotations can be used to differentiate the various dance movements. In order to have more control over the choreographed dance, a 3D animation is preferable. During the past decade, evolution of sketch-based interfaces has provided 3D interaction techniques for modelling and animation. Long traditions of mouse-keyboard-menu-based techniques are now taking more intuitive forms. However, there is no significant effort on creating a sketch-based solution for dance choreography.

This thesis presents a novel sketch-based approach to assist dance choreographers authoring dance motions in a 3D environment. The proposed approach allows a choreographer to storyboard a dance using stick figure sketches of a dancer. It also helps to draw the trace of a 3D avatar to direct it in the same way choreographer intends. Inspired by traditional choreography, a set of simple annotations is introduced for ballet. These annotations help to retrieve and blend ballet 'mini-motions' in order to create a synthesized dance. To build the mini-motions, several ballet movements in a MoCap database are analyzed and processed.

The proposed approach is unique in its use of captured motions, an intuitive method for motion control and its ease of use for non-experts. It allows choreographers to convey their ideas using an animation sequence. The ability of watching dance motions from different angles helps to exactly plan the scene. This approach has the potential to revolutionize the way choreographers create dance patterns.
\end{abstract}




\section{Acknowledgements}

First and above all, I want to thank god for enlightening all aspects of my life with his majestic presence. I am grateful to have the most caring supervisor ever, Professor Richard Levy, who was always there to help me regardless of the circumstance. This research could not be finished without his unconditional encouragement, support and motivation throughout my graduate studies. I also want to express my sincere gratitude to my co-supervisor, Professor Faramarz Samavati, for his regular guidance and support over the course of this research. I could not have done it without the aid of his wisdom, creativity and source of knowledge. Also, I would not be here without the endless help of my husband, Dr. Javad Sadeghi, who has been my best friend and motivation. Thank you my love, for believing in me, supporting me and most importantly pushing me to be all that I can be. I consider myself very lucky to have a super-genius intellectual like you in my life.

It has been my privilege to work closely with Professor Gerald Hushlak since the beginning of my graduate studies. I have gained a lot of experience working with him on different projects. He has been a kind supporter, a creative collaborator and a great friend for me and my family. I have always been blessed to be raised in a lovely and educated family. I can not put into words the amount of support that I received from my father, Professor Mohammad Reza R. Moghaddam. He is the most incredible, humble, talented and generous man I have ever known. He has been a great role model, teacher and idol for me and many others. Thank you dad

for believing in me and teaching me how to dream big. I can never thank enough from my mother, Rafat Hosnirokh, for everything she has done to make sure my life 
turns out perfectly. Thanks mom for your worries, endless concerns and life-changing lessons. I always respect you for being the first person who taught me how to read and write. You are the smartest, the most independent and the bravest woman I have ever known. I love you very much and will never forget your lesson that by endeavor and perseverance I can achieve all of my goals.

Being the youngest child in a family has a lot of benefits and I am the luckiest girl for having two older sisters: Azadeh and Elham. I have learned a lot from both of my smart, kind and beautiful sisters. You are my best friends no matter how old I am or where I am, and I will never forget my childhood memories with you. Also, I can not explain the amount of care that I have received from my father in-law, Hassan Sadeghi, and mother in-law, Fatemeh Sadeghi. Thank you for your weekly calls and reminding the value of belief and importance of education to me. I also would like to thank my brother-in-laws, Ali Hashemi and Dr. Mohammad Reza Pourhaghighi and their lovely angels Arad Hashemi and Adrina Pourhaghighi. I am the happiest auntie in the world for having the nicest nephew and niece ever. I am also grateful to my sister in-laws Masoomeh and Zahra Sadeghi for always being kind to me. Last but not least, I believe that the spirit of my late brother in-law, Reza Sadeghi, was always inspiring, motivating and promoting me toward performance excellence. 


\section{Table of Contents}

Approval Page ii

Abstract iii

Acknowledgements iv

Table of Contents vi vi v

$\begin{array}{lll}\mathbf{1} & \text { Introduction } & \mathbf{1}\end{array}$

1.1 Motivation . . . . . . . . . . . . . . . . . . . . . 4

1.2 Goals . . . . . . . . . . . . . . . . . . . . . . . . 5

1.3 Problems. . . . . . . . . . . . . . . . . . . . . . . 6

1.4 Methodology . . . . . . . . . . . . . . . . . . . . . . . . . 9

1.5 Contributions . . . . . . . . . . . . . . . . . . . . . . . . . . . 11

1.6 Overview of Thesis . . . . . . . . . . . . . . . . . . . . 12

2 Background and Related Work 13

2.1 Dance . . . . . . . . . . . . . . . . . . . . . . . . 13

2.2 Ballet . . . . . . . . . . . . . . . . . . . . . 16

2.3 Ballet Styles . . . . . . . . . . . . . . . . . . . . . . . . . . . . . . . . 19

2.3 .1 Classical Ballet . . . . . . . . . . . . . . . . . . . 20

2.3 .2 Neoclassical Ballet . . . . . . . . . . . . . . . . . 20

2.3.3 Contemporary Ballet . . . . . . . . . . . . . . . . . 21

2.4 Ballet Poses and Ballet Motions . . . . . . . . . . . . . . . . . . . . . 22

2.5 Ballet Master . . . . . . . . . . . . . . . . . . . . . . . . . . . . . 23

2.6 Basic Foot Positions . . . . . . . . . . . . . . . . . . . . . 25

2.7 Choreography . . . . . . . . . . . . . . . . . . . 27

2.8 Dance Annotation . . . . . . . . . . . . . . . . . . . . . . . . . . . . . 29

2.9 Motion Capture Technique (MoCap) . . . . . . . . . . . . . . . . . . 30

2.10 Related Work . . . . . . . . . . . . . . . . . . . . . . . 32

\begin{tabular}{lll}
\hline & Annotation Analysis & 39
\end{tabular}

3.1 Dance Annotation . . . . . . . . . . . . . . . . . . . . . . . . . . . . . 39

3.2 Words and Word Abbreviation Systems . . . . . . . . . . . . . . . . . 40

3.3 Track Drawing Systems . . . . . . . . . . . . . . . . . . . . . . . . . 41

3.4 Stick Figure (Visual) Systems . . . . . . . . . . . . . . . . . . . . . . 42

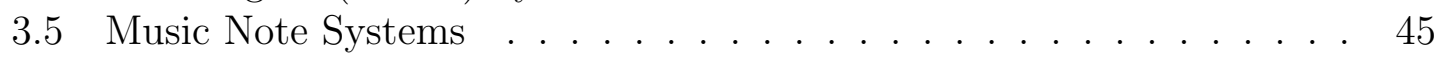




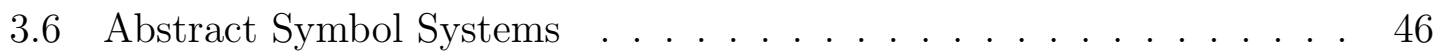

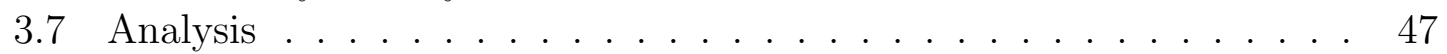

\begin{tabular}{lll}
\hline & Methodology & 50
\end{tabular}

$4.1 \quad$ Field Study $\ldots \ldots \ldots \ldots \ldots$

4.2 Design Process $\ldots \ldots \ldots \ldots \ldots$

4.3 Stick Figure Annotation $\ldots \ldots \ldots \ldots$

$4.4 \quad$ Using Captured Motions . . . . . . . . . . . . . . . . . . . . 61

$4.4 .1 \quad$ Creating Mini-motions . . . . . . . . . . . . . . . . . . . 63

4.5 Sketch Recognition . . . . . . . . . . . . . . . . . . . . . 65

4.6 Sketch-Based Dance Choreography . . . . . . . . . . . . . . . 67

4.6 .1 Limitations . . . . . . . . . . . . . . . . . . . 69

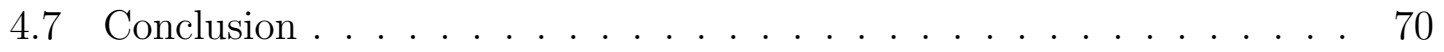

$\begin{array}{lll}5 & \text { Results } & \mathbf{7 1}\end{array}$

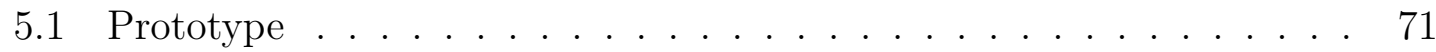

$5.2 \quad$ Applications . . . . . . . . . . . . . . . . . . . . . . . . 76

5.3 Evaluation . . . . . . . . . . . . . . . . . . . . . . . . . . . . . . 77

5.3 .1 Comparison of Feet Position . . . . . . . . . . . . . . . 78

5.3 .2 Comparison of Arm Position . . . . . . . . . . . . . . . . . 81

5.3 .3 Comparison of Jumping Sequence . . . . . . . . . . . . . . . . 83

5.3 .4 Comparison of Walking Sequence . . . . . . . . . . . . . . . 85

5.3 .5 Comparison of Turning Sequence $\ldots \ldots \ldots$. . . . . . . . . 87

5.4 Discussion $\ldots \ldots \ldots \ldots \ldots \ldots$

$\begin{array}{lll}6 & \text { Conclusions } & 92\end{array}$

6.1 Future Work . . . . . . . . . . . . . . . . . . . . . . . . . 93

\begin{tabular}{ll}
\hline Bibliography & 95
\end{tabular} 


\section{List of Figures}

\begin{tabular}{|c|c|}
\hline & the tomb of Nebamun. Image adapted from [36] avail- \\
\hline & ole through Wikimedia Commons. \\
\hline & dance steps on the floor. \\
\hline & Image from [23] available through Wikimedia Commons. \\
\hline 1.3 & Motion Doodles: an interface for sketching character motion. Image \\
\hline & taken from [56]. . \\
\hline 1.4 & nforces standardized foot and arm placements. Image courtesy \\
\hline & of Martha Swope available through Encyclopdia Britannica. \\
\hline 1.5 & An screenshot of the Dance Designer. It allows playback of videos \\
\hline & simultaneously with the choreographed performance. Image taken \\
\hline & from [17]. \\
\hline 1.6 & An screenshot of the DanceForms. Image taken from [15]. \\
\hline 1.7 & The breakdown of the proposed approach for creating a dance se- \\
\hline & uence. Each input sketch is associated with a mini-motion. By \\
\hline
\end{tabular}

\begin{tabular}{|c|c|c|}
\hline 2.1 & Dancing maenad, 330-320 BC. Image from British Museum available & \\
\hline & through Wikimedia Commons. . & 14 \\
\hline 2.2 & Persian women dancing. From a wall painting at "Hasht Behesht & \\
\hline & Palace" (Palace of eight heavens) in Isfahan, Iran available trough & \\
\hline & Wikimedia Commons. & 15 \\
\hline 2.3 & Representation of a ballet for Henri III and his court. Image taken & \\
\hline & from the Gallery of Louvre available through Wikimedia Commons. & 17 \\
\hline 2.4 & King Louis XIV as Apollo, god of sun, in Le Ballet de la Nuit. Image & \\
\hline & sy of Henri Gissey (1621 - 1673) available through Wikimedia & \\
\hline & Commons. & 18 \\
\hline 2.5 & The Nutcracker ballet dance work. Image taken from the first perfor- & \\
\hline & Nutcracker in Mariinsky Theater at 1892 by anonymous Rus- & \\
\hline & sian $19^{t h}$ century photographer, available through Wikimedia Commons. & 19 \\
\hline 2.6 & es: (a) Arabesque (b) Attitude and (c) Croisée. & \\
\hline & s from the American Ballet Theatre's online ballet dictionary [55]. & 2 \\
\hline 2.7 & reenshots of the Avant mini-motion. Images from & \\
\hline & the American Ballet Theatre's online ballet dictionary [55]. & 23 \\
\hline & shots of the Arriére m & \\
\hline & arv 55. & \\
\hline
\end{tabular}


2.9 Pierre Beauchamps, a Ballet Master at the Royal Academy of Dance, is credited with developing the five basic foot positions of ballet. Image taken from [23]. . . . . . . . . . . . . . . . . . . . . . . . . . . . . . 24

2.10 First position of the foot in ballet. Image (a) taken from [62]. Image (b) taken from Wikimedia Commons. . . . . . . . . . . . . . 25

2.11 Second position of the foot in ballet. Image (a) taken from [62]. Image (b) taken from Wikimedia Commons. . . . . . . . . . . . . . . 26

2.12 Third position of the foot in ballet. Image (a) taken from [62]. Image

(b) taken from Wikimedia Commons. . . . . . . . . . . . . . . 26

2.13 Fourth position of the foot in ballet. Image (a) taken from [62]. Image (b) taken from Wikimedia Commons. . . . . . . . . . . . . . . . 27

2.14 Fifth position of the foot in ballet. Image (a) taken from [62]. Image (b) taken from Wikimedia Commons. . . . . . . . . . . . . . 28

2.15 In motion capture, markers and special cameras are used to record a real movement. Image published under GNU License at Wikimedia

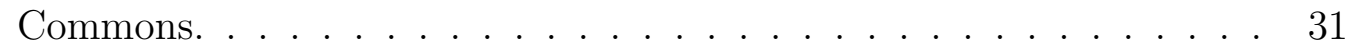

2.16 A user interface for representing the animation of a Labanotation score. Image taken from [15]. . . . . . . . . . . . . . . . 33

2.17 A screenshot of the Web3D Dance Composer. Image taken from [52]. 34

2.18 An illustration of a Motion Graph. Image taken from [37].] . . . . . . 36

2.19 Drawn keyframes of a throw motion and the final 3D representation. Image adapted from [19]. . . . . . . . . . . . . . . . . . . 37

2.20 An illustration of the MotionMaster. Image adapted from [41]. . . . . 38

2.21 Comparison of the major related works to this research based on their

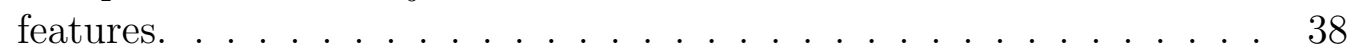

3.1 Ballet dance notation systems. . . . . . . . . . . . . . . . . 40

3.2 An example of word abbreviation notation in LÁrt et instruction de bien dancer. Image taken from [50]. . . . . . . . . . . . . . . . . 41

3.3 An example of Feuillet annotation based on track drawing for The Bourée dÁchille. Image from [23] available through Britanica. . . . . . 42

3.4 Extracts from the score of Fanny Elssler's Cachucha choreographed using Zorn's system. Image from [62] available through Wikipedia. . . 43

3.5 An example of recorded movements in Benesh movement notation. Image taken from Royal Academy of Dance website [45]. . . . . . . . 44

$3.6 \quad$ A sample choreography using Stepanov's notation. Image available in the public domain through Wikimedia commons. . . . . . . . . . . . 45

3.7 Laban's notation system. Image taken from [39]. . . . . . . . . . . . 46 
3.8 An example of the dance notation system developed by Noa Eshkol and Abraham Wachmann. Image available in the public domain through Wikimedia commons. . . . . . . . . . . . . . . . 47

$4.1 \quad$ Pirouette mini-motion. Image taken from [47]. . . . . . . . . . . . . . 53

4.2 Pirouette mini-motion played by two different dancers. Images taken from [14]. $\ldots \ldots \ldots \ldots \ldots$

4.3 An early concept for the user interface of the proposed approach. . . 55

$4.4 \quad$ A sample storyboard for sketch-based dance choreography. . . . . . . 56

$4.5 \quad$ A sample storyboard for sketch-based dance choreography. . . . . . . 57

$4.6 \quad$ A sample storyboard for sketch-based dance choreography. . . . . . . 58

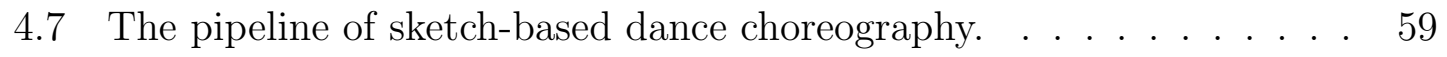

4.8 Pirouette (a) in Zorn Annotation and (b) in new annotation. . . . . 59

4.9 Jeté (a) in Zorn Annotation and (b) in new annotation. $\quad$. . . . . . . 60

4.10 Rond de jambe (a) in Zorn Annotation and (b) in new annotation. . 60

4.11 List of the main stick figure annotations proposed for this work. . . . 61

4.12 Characterization of the raw MoCap data in Motion Builder. . . . . . 62

4.13 Re-targetng the motions to a rigged model. . . . . . . . . . . . . 63

4.14 The pre-processing pipeline. . . . . . . . . . . . . . . . . . . . 63

4.15 The Jeté mini-motion prepared according to the annotations provided in Figure 4.9(a). . . . . . . . . . . . . . . . . . 64

4.16 The various ways of drawing a square do not matter in the resulting

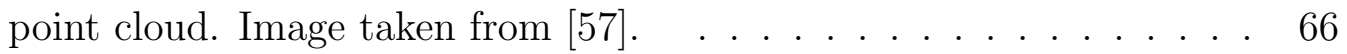

4.17 A sequence of dance following the sketched trajectory. . . . . . . . . 67

4.18 To align the direction of the dancer on the path's current direction, the dancer is tilted towards the approximate tangent. . . . . . . . . 68

4.19 Unity3D's state machine is used to blend different mini-motions. . . 69

4.20 Blending two mini-motions using linear interpolation. . . . . . . . . 69

5.1 The user interface of the first prototype created for this thesis. . . . . 72

$5.2 \quad$ The process of adding mini-motions to the movement list. . . . . . . . 73

5.3 The process of changing the order of attitude mini-motion in the movement list and removing it from the list. . . . . . . . . . . . . . . . 73

5.4 The user interface of the final prototype created for this thesis. . . . . 74

5.5 The sketch-based process for adding mini-motions to the movement list. 75

$5.6 \quad$ The process of directing the dancer by drawing a trajectory. . . . . . 76

5.7 The Attitude pose shown from different angles. . . . . . . . . . . . . 77

5.8 Combination of Arabesque, Turn step and Folding arms mini-motions. 77

5.9 Combination of Quasi cou-de-pied, Raised leg above hip height and Jete en touran mini-motions. . . . . . . . . . . . . . . . . . . . . 78 
5.10 Combination of Sideways steps and Pirouette mini-motions. . . . . . 79

5.11 Comparing feet position between the common annotation systems. . . 80

5.12 Comparing arm position between the common annotation systems . 82

5.13 Comparing Jumping Sequence between the common annotation sys-

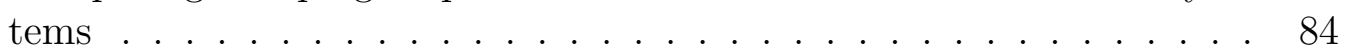

5.14 Comparing walking sequence between the common annotation sys-

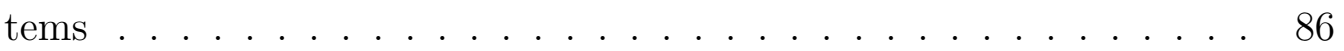

5.15 Comparing Turning sequence between the common annotation systems ...................... . . . . . . . . . . . . .

5.16 Comparison of the Sketch-Based Dance Choreography with major related works based on their features. . . . . . . . . . . . . . . . . . . . 91 


\section{Chapter 1}

\section{Introduction}

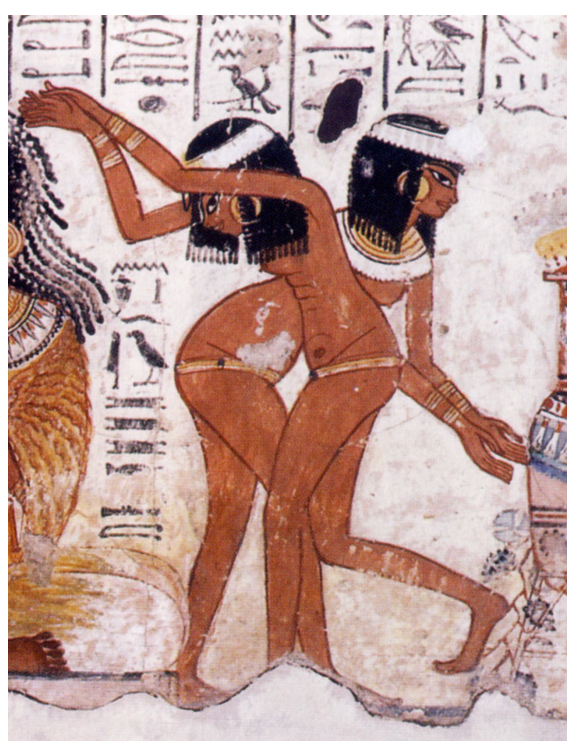

Figure 1.1: Dancers from the tomb of Nebamun. Image adapted from [36] available through Wikimedia Commons.

Dance is an art form that involves rhythmic movements of the body. These movements are usually synchronized to a musical score and sometimes used to express an idea or story. Dance is used in many cultures as a form of emotional expression, social interaction, or exercise [4]. It is difficult to determine when dance became a part of human life. Archaeologists have found traces of dance in India from 9,000 years ago and in Egypt from 3300 BC [59]. Figure 1.1 shows an ancient painting of dancers from tomb chapel of Nebamun in Thebes, Egypt at $14^{\text {th }}$ century BC [36]. Because of the lack of specific knowledge about the origin of dance, many dance experts relate the history of dance to the ritual dances preserved in tribal societies. 
Dance as a social activity and a form of entertainment became popular in mediaeval Europe. Many dances were developed during the Middle Ages as a feature of court festivities. Ballet, which is the centre of focus in this thesis, was one such dance. It was originated from the court dances in Italy and France between $15^{\text {th }}$ and $16^{\text {th }}$ centuries [11].

Since the 1400s, the court dances were detailed and complicated and it was necessary to document the steps to preserve their consistency. With the popularity of ballet in France, special dance instructors or "Ballet Masters" began to appear [5]. They developed choreographic techniques to design dance patterns to be used throughout Europe. Dance choreography (also known as dance composition) is the act of composing dance patterns, usually intended to be performed in a concert [12]. Historically, choreographers have used drawings with a set of annotations to compose different dance patterns (see Figure 1.2). The initial patterns are then observed and rehearsed several times to find any problems and correct them [27]. Annotations play an essential part in this process, as they help document and preserve this traditional art form.

Over the decades, more than twenty annotation systems have evolved for dance choreography, which can be divided into five categories: word abbreviations, track drawings, stick figure (visual) systems, music note systems and abstract symbol systems [29]. Using the word abbreviation of the movements were popular in the early manuscripts of the $15^{\text {th }}$ century [7]. The idea is still employed for dance forms with an established vocabulary of steps. Another older annotation system that dates back to the $17^{\text {th }}$ century is based on tracing the foot or body of the performer on the floor [23]. As shown in Figure 1.2, this technique usually accompanies the floor plan 


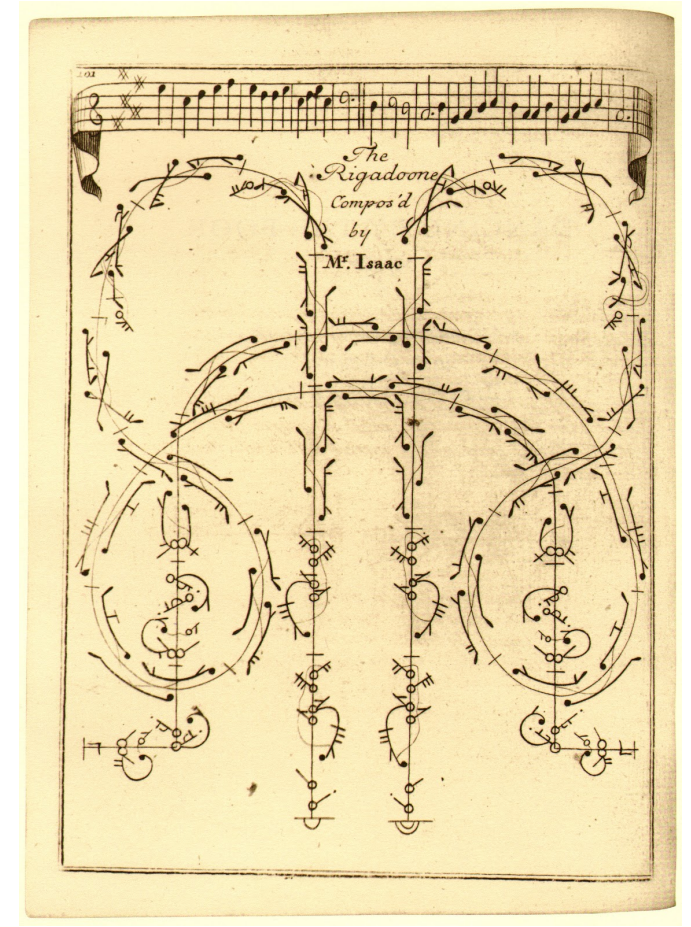

Figure 1.2: Feuillet technique for choreography traces dance steps on the floor. Image from [23] available through Wikimedia Commons.

with a melody line. The stick figure systems [62, 10] are easy to read and have fewer signs compared to the other annotation systems. The music note annotations [53] use music staff to indicate the timing and duration of the dance. This is helpful for people with strong musical background. The abstract symbol systems [39, 22] consider a detailed symbolic language which is usually time-consuming to draw.

In short, the classic word based annotations [7] and track drawing systems [23] are simple to use but not rich enough for the full control of 3D dance movements. Other, rich but complicated 2D annotations (e.g. labanotation [39]) are difficult to understand for novice dancers (see section 3]. Only stick figure systems [62, 10] provide a good balance between richness and simplicity. 


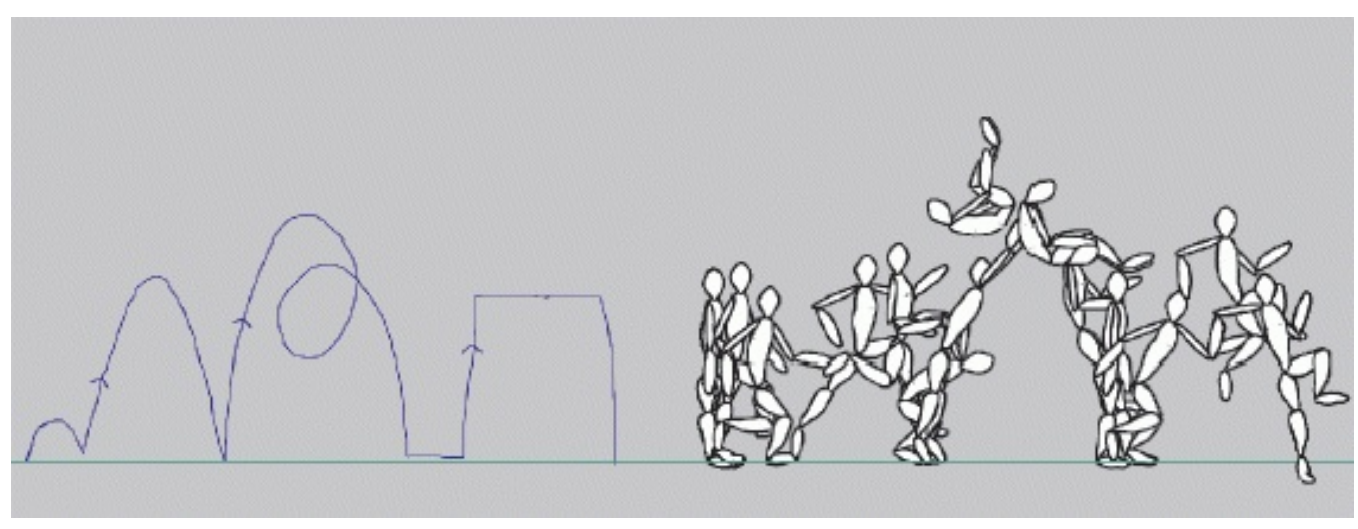

Figure 1.3: Motion Doodles: an interface for sketching character motion. Image taken from [56].

\subsection{Motivation}

Living in today's digital world, one might ask: is it possible to use simple 2D annotations to unambiguously identify 3D dance movements? With this approach, the traditional process of dance choreography can be accompanied by a dancing avatar. This approach could help choreographers view the resulting dance from an arbitrary angle that reduces the ambiguities related to the dance patterns.

Recently, there has been increasing interest in sketch-based interfaces for creating and controlling animation. These interfaces have caused a shift in the field of 3D animation interfaces by providing a more natural means of interaction. Long traditions of mouse-keyboard-menu-based techniques are now taking more intuitive forms. Figure 1.3 shows such an interface that controls the motion of a character using simple strokes [56]. While controlling the full range of human motions with a small set of simple annotations is a challenging problem, ballet consists of limited and constrained motions (see Figure 1.4). This motivated the current research on providing a sketch-based approach to create ballet dance animations in a 3D 

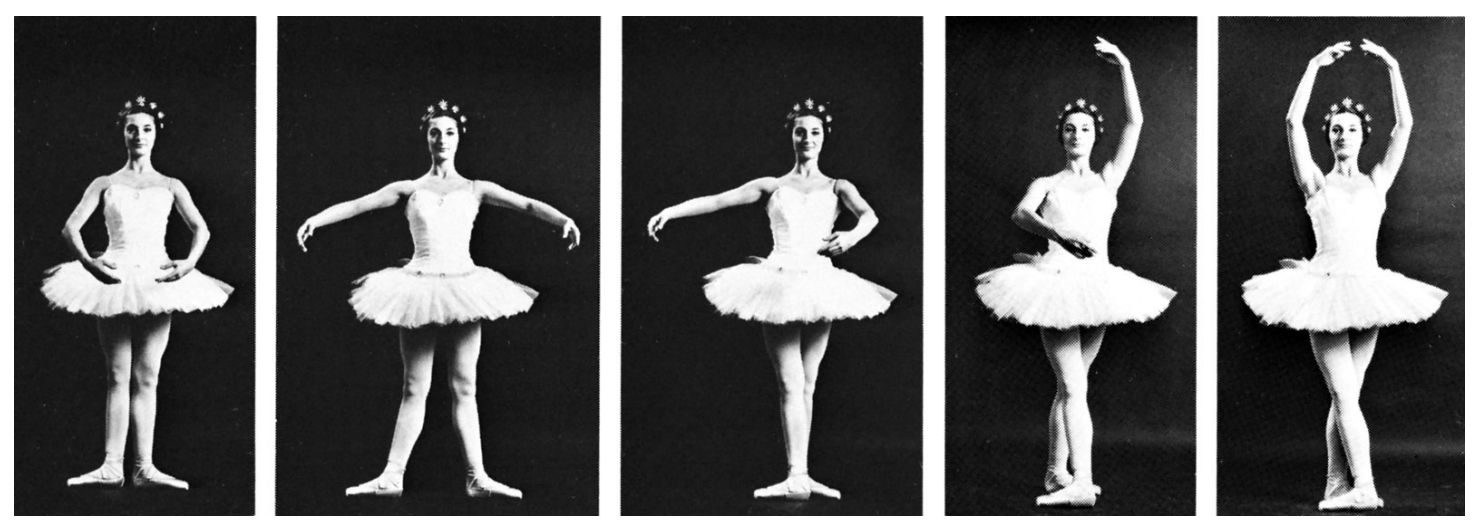

Figure 1.4: Ballet enforces standardized foot and arm placements. Image courtesy of Martha Swope available through Encyclopdia Britannica.

environment.

Another motivation for this research was to facilitate teaching ballet to everyone at any skill level. Learning ballet has always been challenging specifically for children. Having a 3D choreography environment that allows saving the final dance sequence as a 3D animation can help learning ballet. Specifically, it is motivating for children because they usually like watching animated characters.

\subsection{Goals}

The main goal of this research is to provide a new sketch-based approach for dance choreographers to intuitively design and animate ballet sequences. As highlighted in section 1.1, using 3D avatars helps to avoid possible ambiguities of $2 \mathrm{D}$ choreography by adding extra details to the dance movements. Currently, there is no 3D approach for dance choreography that allows authoring dance patterns in the same way choreographers used to work on a piece of paper. This thesis aims to provide such a sketch-based approach for designing and editing different dance patterns in a 
3D environment. These are the main objectives that desired approach should satisfy:

- Provide an easy to follow and comprehensive annotation system.

- Enhance the 2D process of choreography by designing a 3D environment for choreographers.

- Facilitate authoring and editing dance patterns in the same way done by choreographers.

- Synthesize 3D motions using captured sequences of the real performances.

The current annotation systems that are used in ballet choreography tools are usually complex and difficult to be understood by every choreographer. The goal of this work is to make it possible for any choreographer or even novice dancer to compose a detailed ballet sequence. This can be achieved by providing a simplified dance annotation system that makes sure to record all of the details of a real performance using a set of canned animations.

\subsection{Problems}

To help choreographers design, edit and communicate different ballet patterns with all of their details in a 3D environment, an easy to follow yet comprehensive annotation system is necessary. While most people can figure out track drawing systems for ballet, only specialists can learn and use abstract annotations correctly. The main reason is that, these annotations can only be simplified to a certain degree otherwise, it will not be possible to show detailed 3D information using them. Only the stick figure systems are easy to read and have less ambiguity. 


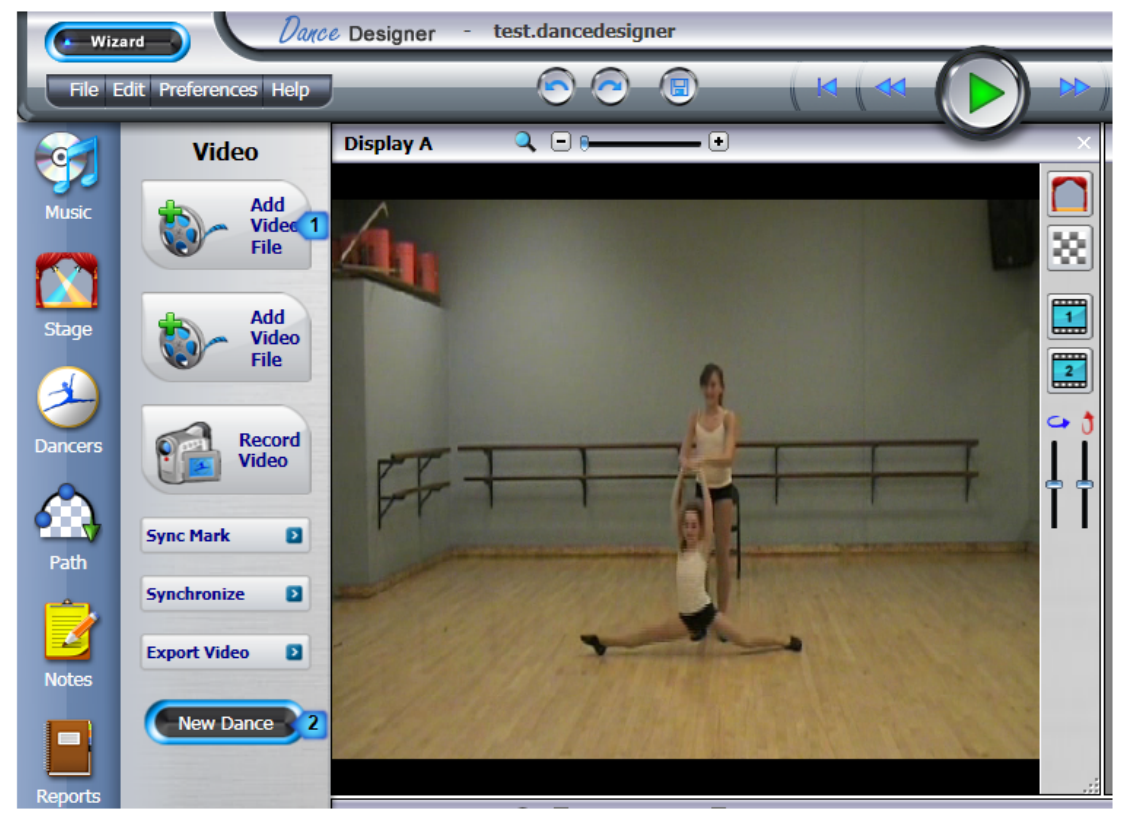

Figure 1.5: An screenshot of the Dance Designer. It allows playback of videos simultaneously with the choreographed performance. Image taken from [17].

While a good stick figure annotation system helps to document the dance in a more readable format, it can not necessarily represent all of the subtle movements of the performer in the 3D space. A common approach for enhancing dance composition is videotaping the dancers using ubiquitous video capturing devices. Both choreographers and dancers can benefit from this approach and watch the captured movements later to decipher complex dance steps. Dance Designer [17] is a commercial tool that uses this idea to assist choreographers with two videotaped dances (see Figure 1.5). While the ease and speed of the video learning enhances the 2D annotations, the videotaped dance is not sufficient to provide all the $3 \mathrm{D}$ information. The main reason for this limitation is that consumer cameras can not capture the depth of the objects in the scene. 


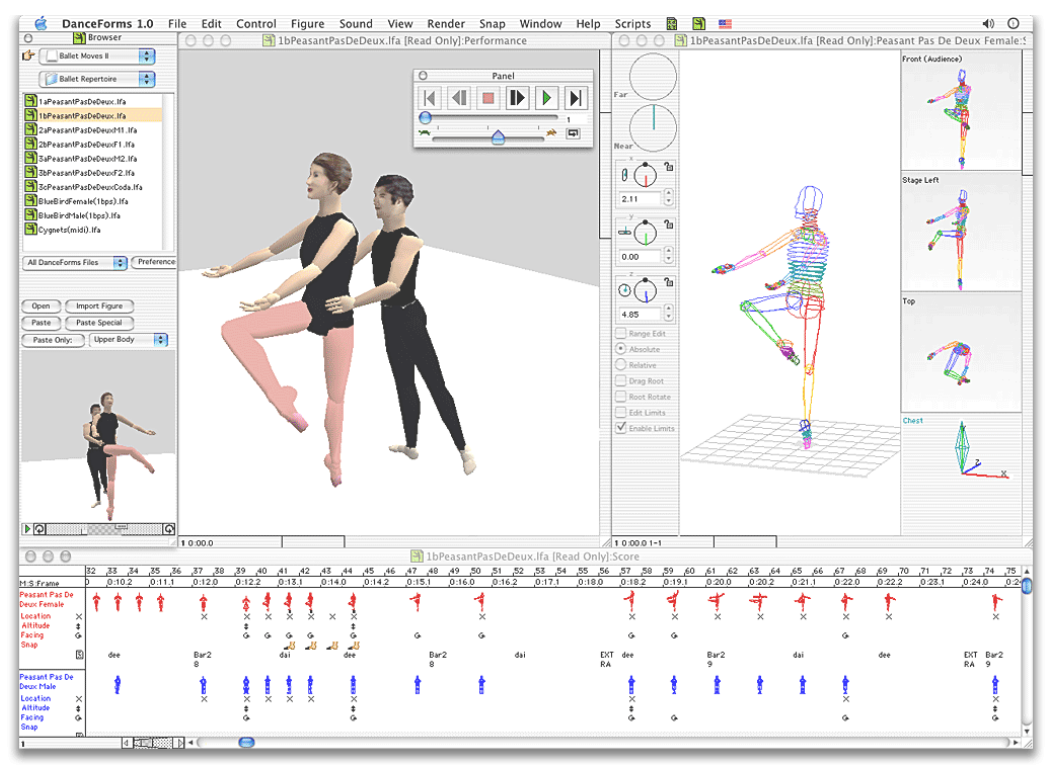

Figure 1.6: An screenshot of the DanceForms. Image taken from [15].

As noted in [28], during videotaping a dance, some parts of the movements may get hidden by the dancer which enforces taking more videos from other angles. Therefore, to envision a 3D dance pattern it should be recorded and replayed concurrently from numerous angles which is not usually affordable. Also, if the videotaped dance is not what choreographer intends, it is not yet possible to correct the steps on the video. In addition, in a large rehearsal it is not easy to capture all of the details of each dancer using videotape. Therefore, videotaped scenes are not sufficient to communicate all the details of the 3D dance pattern envisioned by the choreographer.

Showing the dance in a 3D environment similar to DanceForms [15] can disambiguate the spatial information in 2D choreography (see Figure 1.6). However, DanceForms does not allow choreographers to design dance patterns in the same way they sketch them on a piece of paper. In addition, the 3D motions generated with DanceForms are artificial and do not have all the details of the real performance. 
This is mainly resulted from the limitation of DanceForms' dynamic animation generation. Creating realistic human motion with all of the natural movements is challenging, because human motion has a lot of small details that should be considered in animation.

The problems discussed here, show a demand for a sketch-based choreography approach that can preserve all of the details of a real performance in 3D.

\subsection{Methodology}

In order to design a method for achieving the research goals, first, a few ballet masters and choreographers were debriefed informally about the tools and techniques used by them and their limitations. They highlighted the importance of using simpler annotations that keep the artistic nature of the dance instead of showing it using rigorous formula. To satisfy this requirement, the history of annotation systems was explored which provided good inspirations for the proposed annotation system. These domain experts also complained about the flaws of the current video-based approach for communication between dancers and choreographers. To avoid these limitations, a 3D environment was chosen for the proposed approach. Finally, they recommended designing an approach to simulate what dancers do in the real world. Dancers usually combine different mini-motions together to create a dance pattern. The proposed approach benefits from this idea to simulate the dance motions in the same way that happens in reality.

The above-mentioned design decisions help address the problems described in section 1.3 by assisting dance choreographers authoring and editing dance motions 
in a 3D sketch-based environment. The advantage of using a $3 \mathrm{D}$ environment is that choreographers can see the designed dance as an animated character. Also, the input interface is more closely related to the traditional media (pen and paper) used for choreography than the standard mouse-and-keyboard interface. This makes it possible to use this approach on tactile devices and interact with them similar to paper. For implementation of this approach, Unity3D game engine was chosen because it provides a powerful platform for dynamic motion creation.

As illustrated in Figure 1.7, doodling a rough sketch in the proposed approach can trigger a ballet mini-motion. These mini-motions cover the basic movements of ballet, hence are strongly constrained. To represent and retrieve these minimotions successfully, a simple set of stick figure annotations are introduced. This new annotation system simplifies different moves to just one single stick figure. As discussed in section 1.3 , creating realistic human motions over the full spectrum of natural movements is challenging because human motion features many subtle details that should be considered in animation. Motion Capture (MoCap) techniques [25] can address this issue by providing 3D animation clips with all the characteristics of a live performance.

To construct mini-motions, the ballet motion clips from a MoCap database are segmented according to the poses defined in a standard stick figure annotation system. A state of the art sketch recognition algorithm is used to associate the input sketch to the corresponding mini-motion. The retrieved mini-motions are then added to a list that can be re-ordered for the best combination. Choreographers are able to sketch the trace of the dancer in the scene as they prefer. By means of the responsive and real-time motion blending mechanism of Unity3D, these mini-motions 


\begin{tabular}{|l|l|l|l|l|}
\hline Input annotation & Glissade devant & Arabesque \\
Thini-motion
\end{tabular}

Figure 1.7: The breakdown of the proposed approach for creating a dance sequence. Each input sketch is associated with a mini-motion. By blending these mini-motions the entire sequence is synthesized.

are smoothly stitched together along this path to synthesize a complete dance. The proposed approach allows playing a music score with the sequence to help choreographer better compose the dance steps. Choreographer can play and replay the chosen score while placing different motions in the sequence to find the best motions that are in sync with the given music. Finally, the proposed approach supports saving the complete choreography as an animation clip for training purposes.

\subsection{Contributions}

The sketch-based approach proposed in this thesis is unique in its intuitive way of motion control, the new set of annotations provided for ballet and its ease of use for non-experts. It not only saves the choreographers' effort, but also helps them to convert their ideas into an animation sequence. In fact, it plays the role of a story boarding tool for them which can help them compose innovative dance patterns. These research contributions can be summarized as:

- The concept of sketch-based dance choreography: an interactive approach for 
ballet composition.

- Analyzing the dance notations used in choreography in order to define a set of simple annotations.

- Preparing a set of captured mini-motions for ballet to be blended smoothly along a given trajectory.

An important advantage of this work is that it allows people to watch the dance steps from different angles and exactly visualize the scene. This facilitates teaching ballet to everyone at any skill level. A prototype is also designed for this work. A computer scientist, contributed in the implementation of this prototype.

\subsection{Overview of Thesis}

This thesis is organized as follows: Chapter 2 provides a brief background of the topics related to the sketch-based dance choreography. It also reviews the important previous work related to each area. Chapter 3 describes the main annotations of ballet and analyses their pros and cons. Chapter 4 outlines the framework of the proposed approach. Chapter 5 demonstrates the advantages of using proposed approach with several examples. Finally, chapter 6 concludes the thesis and discusses interesting future directions.

In this thesis, some of the text and figures of Moghaddam et al. [44] are incorporated. 


\section{Chapter 2}

\section{Background and Related Work}

This chapter reviews the background of the dance, ballet and choreography and introduces the necessary concepts related to the sketch-based approach for dance choreography. First, the history of the dance and ballet are reviewed and different ballet styles are introduced. Then, the dance choreography and different dance annotations are briefly explained. Next, the motion capture technique and its applications are discussed. Finally, major works related to the dance choreography and sketch-based control of animation are discussed.

\section{$2.1 \quad$ Dance}

The art of moving the body according to a musical rhythm is called dance. It may be solo, with a partner or as a group. Each culture has its own interpretation of dance which makes different dance styles. People may use dance to express their ideas, telling a story or simply pleasure of the movement itself. They may also look at dance's emotional, social or physical aspects. In general, dance is one kind

of nonverbal communication between human beings. Even before the birth of the earliest human civilization, dance has been performed in ceremonies, rituals and celebrations. Archaeologists have found paintings and sculptures that show dance has appeared several thousand years ago in ancient India, Egypt and Greece [59]. Figure 2.1 shows a dancing maenad on a skyphos from 330-320 BC in Greece. 


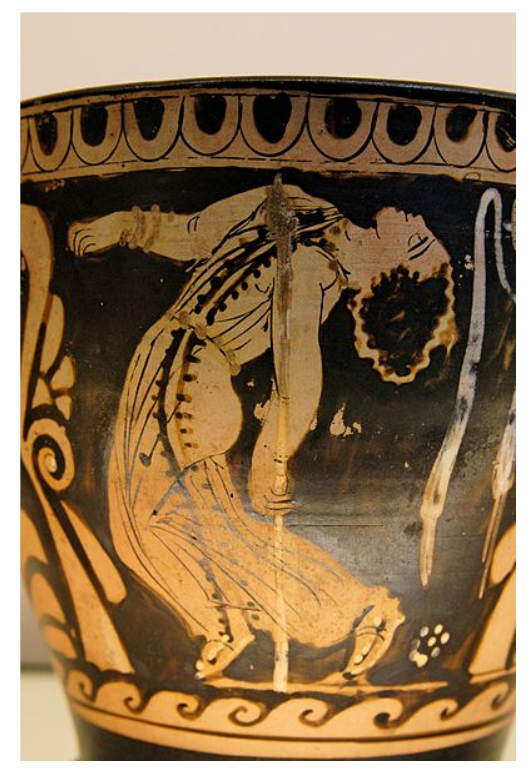

Figure 2.1: Dancing maenad, 330-320 BC. Image from British Museum available through Wikimedia Commons.

One of the earliest documents about dance is the Natya Shastra [58] which was written between 200 BCE and 200 CE in India. It was written in Sanskrit and has been remained as an important text for many centuries. The Natya Shastra divides dance into four categorizes: secular, ritual, abstract and interpretive. It covers a wide range, from music and dance to stage design, makeup and generally every aspect of the stagecraft. In particular, it is a valuable reference for musicians because it is the only text which provides details about the music and instruments of that period.

Before the invention of the writing languages, dance was used to pass the stories down from generation to generation [21]. It was also an early method to show feelings towards the opposite gender. In addition, another early use of the dance was in healing rituals. Medieval Europeans were thought if they dance, it will protect them from diseases. However, these dances sometimes led dancers to death due to 


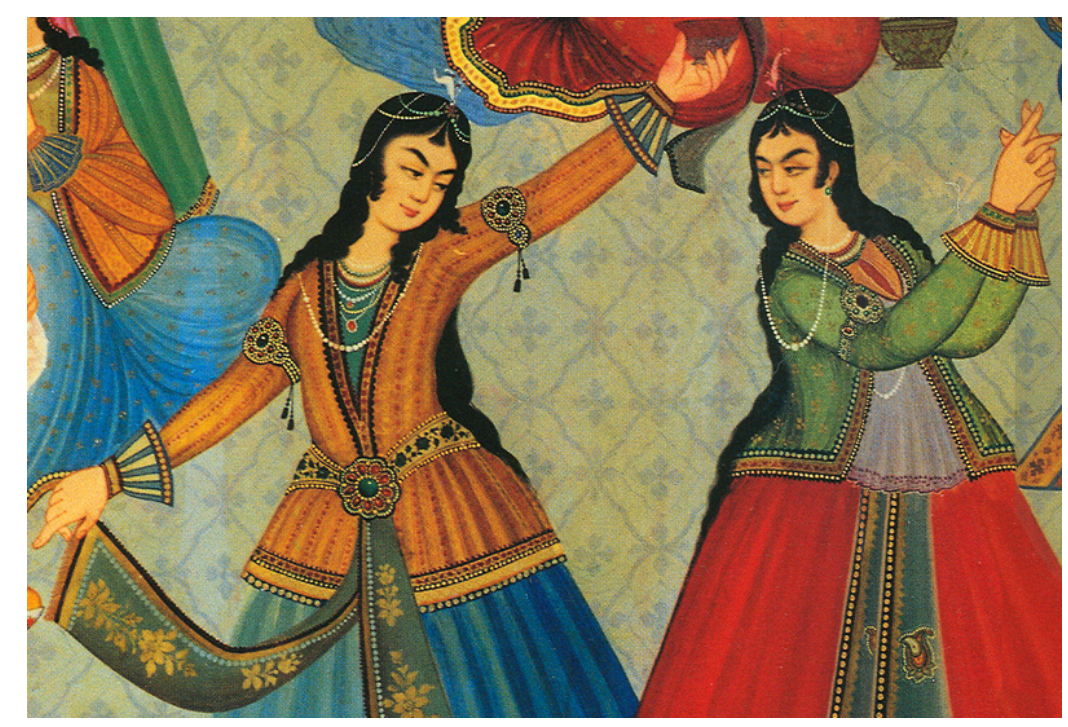

Figure 2.2: Persian women dancing. From a wall painting at "Hasht Behesht Palace" (Palace of eight heavens) in Isfahan, Iran available trough Wikimedia Commons.

the exhaustion. Some tribal cultures still use dance for this purpose.

Many contemporary dance forms root back in the historical, traditional, ceremonial, and ethnic dances. For example, current Persian dance steps are originated from Persian traditional dance style. Figure 2.2 shows a wall painting of a few Persian women dancing in a ceremony in Iran at $17^{\text {th }}$ century. In European culture, one of the earliest records of dancing can be found in Homer's "Iliad", where he describes chorea. The early Greeks made a system to express different passions using the art of dancing. For example, the dance of the Furies could create complete terror for the audience. The Greek philosopher, Aristotle, ranks dancing in the level of poetry. He believes the way dancers apply rhythms to the gestures, can be used to express manners, passions, and actions. However, the history of expressing thoughts and emotions through movements is even older than development of the words used in poetry. 


\subsection{Ballet}

The origins of ballet can be traced back to the Renaissance period and the early court dances in France and Italy [16]. Court dancing at that time was a feature of celebratory occasions, such as marriage ceremonies. The residents of the court learned these dances as part of their training. France's court was in many ways ahead of the other royal courts in Europe. In addition to France, Italy had an important influence on the art forms including ballet. In fact, ballet was first introduced to France's court by Catherine de Medici, the Italian wife of King Henry II of France [8]. It has since become a widespread, highly technical form of dance with its own vocabulary based on French terminology. Figure 2.3 shows a facsimile of an engraving on cooper for the "Ballet de la Royne" by Balthazar de Beaujoyeulx staged in Paris in 1581 for the French court.

King Louis XIV of France had a great influence on development of the ballet. He was an enthusiastic dancer known as the "Sun King" because of the role he played at the age of 14 in Le Ballet de la Nuit (The Ballet of the Night). Figure 2.4 shows a portrait of King Louis XIV performing Apollo, god of sun, in this 12-hour ballet. During Louis' reign, two kinds of dances were developed: social dances for the ballroom and theatrical dances for court entertainments [4]. These two forms shared similar steps and styles and both were practiced by the nobility. In 1661, Louis XIV formed the world's first ballet school, the Académie Royale de Danse (Royal Academy of Dance), for creation, refinement and standardization of the new styles of dance. He died at the age 77 in 1715 but his legacy lived on through most of the eighteenth century. 


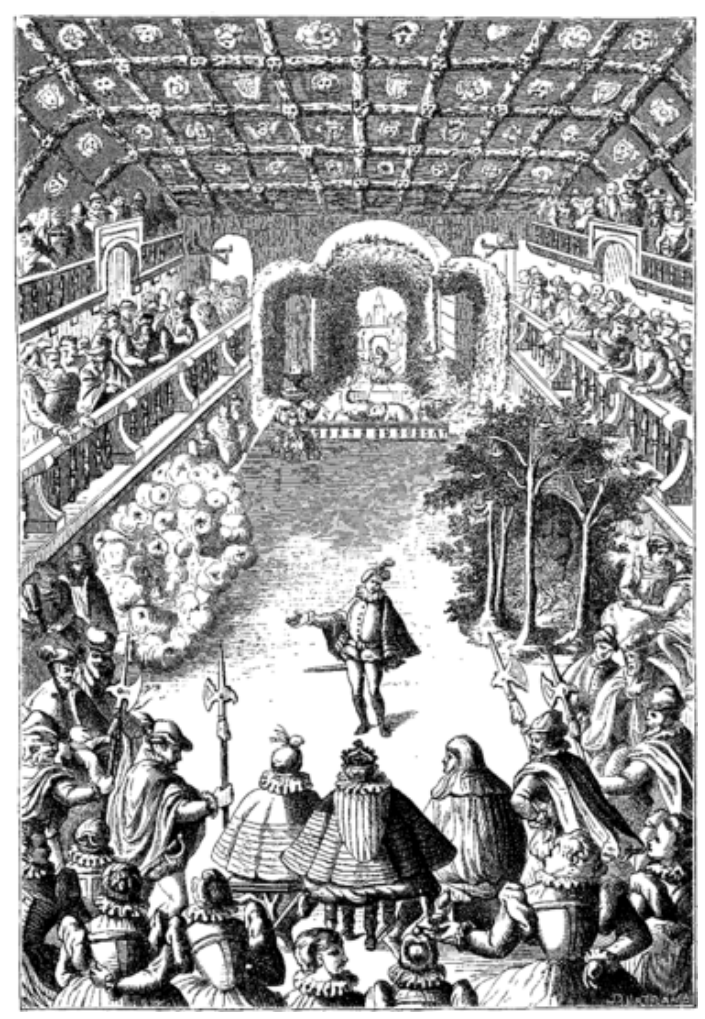

Figure 2.3: Representation of a ballet for Henri III and his court. Image taken from the Gallery of Louvre available through Wikimedia Commons.

During the $18^{\text {th }}$ century, an increasing trend started towards the performance of noble dances by companies of professional dancers. This movement transformed theatrical dance into one of the most refined arts of the time, a tradition that continues today in ballet [18]. In this period, opera was key to ballet. Most operas had to include at least one dance piece. The first four decades of the $19^{\text {th }}$ century were critical times in the history of ballet. During these years, the main aspects of the contemporary ballet were established and dancers were trained and prepared for the new technical challenges. The two most important productions of this era were La Sylphide (Paris, 1832) and Giselle (Paris, 1841) [31.

Ballet requires years of training to learn and master, and much practice to retain 


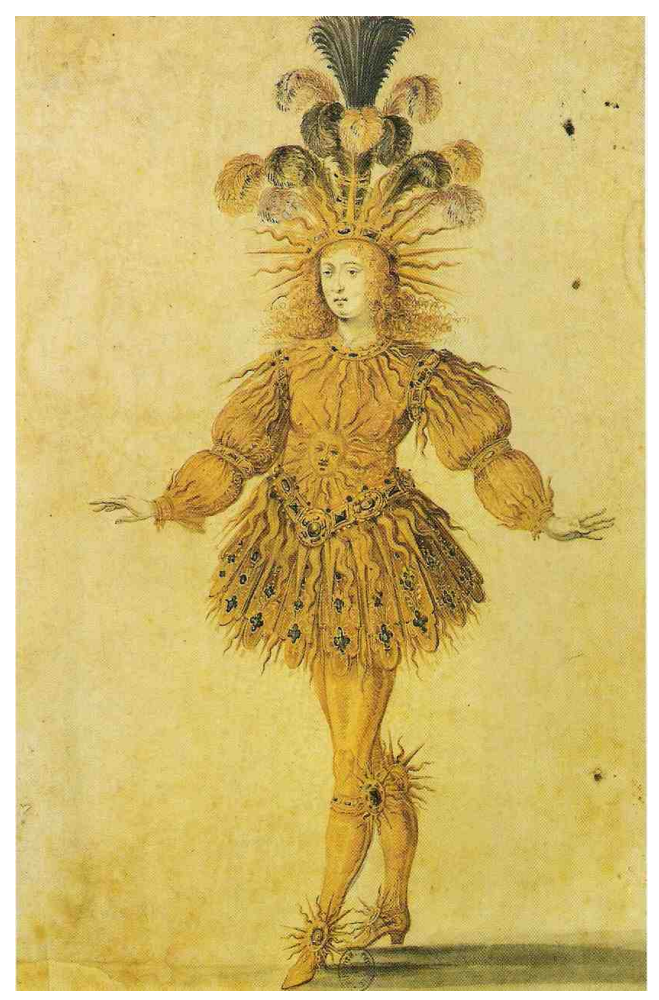

Figure 2.4: King Louis XIV as Apollo, god of sun, in Le Ballet de la Nuit. Image courtesy of Henri Gissey (1621 - 1673) available through Wikimedia Commons.

proficiency. Different ballet schools around the world, have historically used their local culture to evolve the art. Ballet may also refer to a ballet dance work, which consists of the choreography and music for a ballet production. Figure 2.5 represents a well-known example, the Nutcracker, a two-act ballet that was originally choreographed by Marius Petipa and Lev Ivanov with a music score by Pyotr Ilyich Tchaikovsky [32]. Ballet dance works are choreographed and performed by trained artists. Most classical ballet dance works are performed with classical music and are theatrical and may have elaborate costumes and staging. 


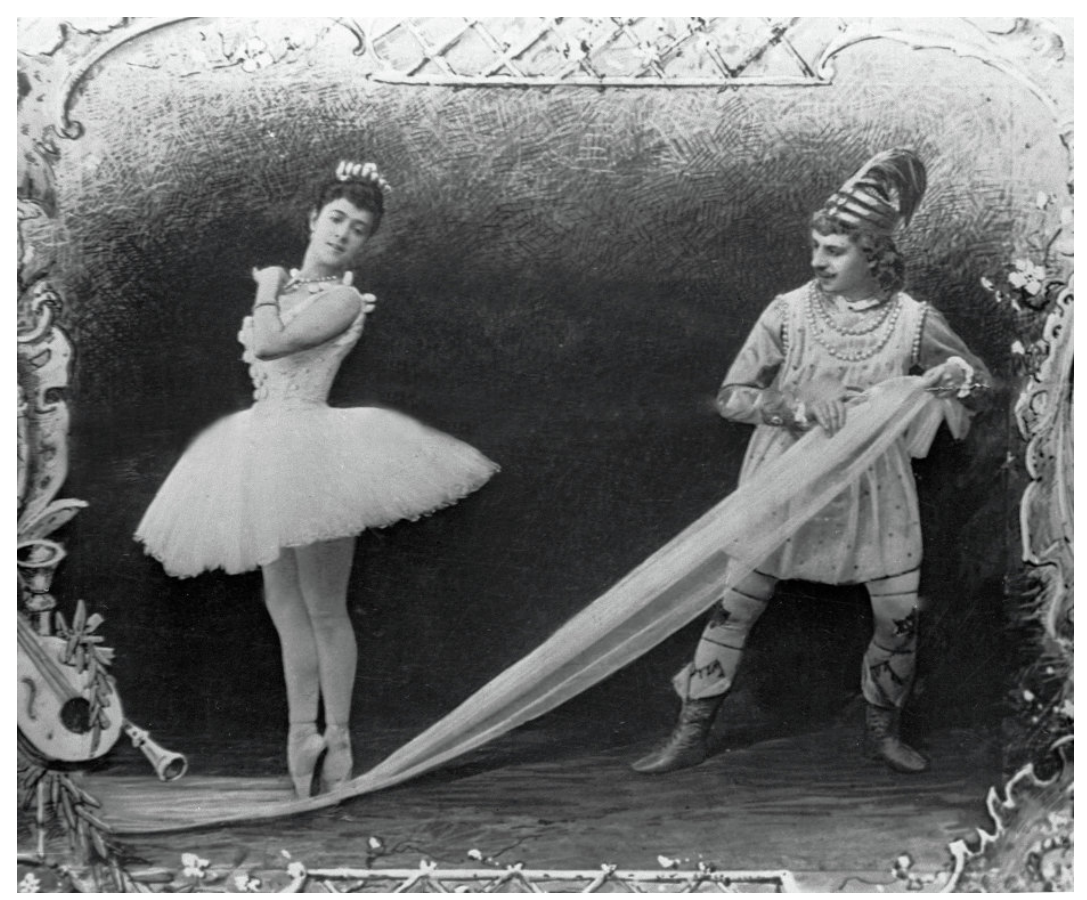

Figure 2.5: The Nutcracker ballet dance work. Image taken from the first performance of Nutcracker in Mariinsky Theater at 1892 by anonymous Russian $19^{\text {th }}$ century photographer, available through Wikimedia Commons.

\subsection{Ballet Styles}

Stylistic variations of ballet have evolved in different geographical regions since the Italian Renaissance. Examples of these styles are Russian ballet, French ballet, and Italian ballet [8]. More recent styles such as contemporary ballet and neoclassical ballet, use both traditional and non-traditional ballet movements. Probably the most widely used ballet style is late Romantic ballet (or ballet blanc) which focuses on pointe work, flowing and precise movements and often presents the female dancers in traditional, short white tutus. Some of the main ballet styles are introduced here. 


\subsubsection{Classical Ballet}

Classical ballet is a formal style of ballet that adheres to traditional ballet techniques and vocabulary. It is known for the flowing, precise movements and use of aesthetics and rigorous techniques (such as pointe work, turn-out of the legs, and high extensions) [5]. There are different variations of the classical ballet based on their areas of origin e.g. Russian ballet, French ballet, British ballet and Italian ballet. Russian ballet features high extensions and dynamic turns, while Italian ballet tends to be more grounded, with a focus on fast and intricate footwork [35]. Some of the classical ballet styles are named after their creators e.g. Cecchetti method is named after its creator, Italian dancer Enrico Cecchetti. Despite their variation, the performance and movement vocabulary of different classical ballet styles are largely consistent throughout the world.

\subsubsection{Neoclassical Ballet}

Neoclassical ballet is a ballet style that uses traditional ballet vocabulary but is less rigid than the classical ballet. It is the $20^{\text {th }}$ century version of the classical ballet appeared first in the works of George Balanchine. The defining characteristic of the neoclassical ballet is its focus on structure. Also its spacing is usually more modern or complex than classical ballet. Balanchine drew neoclassical ballet from the $19^{\text {th }}$ century Russian imperial dance, but striped off its detailed narrative and heavy theatrical setting. What was left was the dance itself, sophisticated but modern, retaining the pointe shoe aesthetic, but avoiding the full length story of the ballet [8].

Balanchine's Apollo in 1928 is considered the first neoclassical ballet. During this

time, Balanchine started a collaboration with modern dance choreographer Martha 
Graham to expand more on modern techniques and ideas. He brought modern dancers into his company, the New York City Ballet, to combine ballet and modern techniques. Instead of using large sets and traditional tutus, his ballet dance works are plotless and musically driven. This simplistic style allows dancers' movements to become the main artistic medium, which is the main attribute of the neoclassical ballet [9].

\subsubsection{Contemporary Ballet}

Contemporary ballet is a new form of dance influenced by both classical ballet and modern dance. It employs the main principles of the classical ballet, but permits a greater range of movements and may not adhere to the strict body lines set forth by schools of classical ballet. Many of the concepts in the contemporary ballet come from the ideas and innovations of $20^{\text {th }}$ century modern dance, including floor work, balletic movements and turn-in of the legs [60]. This ballet style is often performed barefoot and may include mime and acting. George Balanchine is often considered to be the first pioneer of contemporary ballet through the development of neoclassical ballet. Contemporary ballet allows choreographers and dancers a wider range of movement vocabulary for creating and performing work. Today, there are many contemporary ballet companies and choreographers and some of the "classical" companies also regularly perform contemporary works. Most professional contemporary ballet companies base their dance techniques in ballet or modern technique, or a combination of the two, and their repertoires are usually reflective of this diversity. 


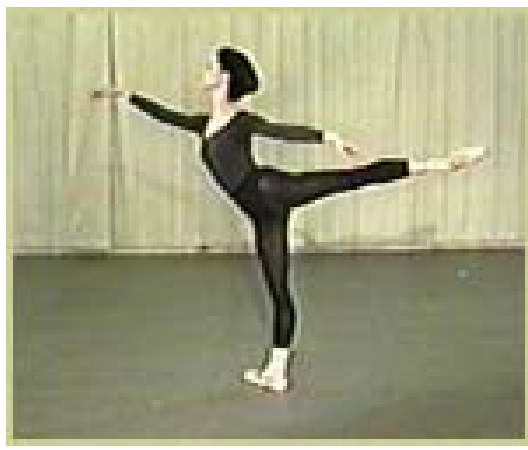

(a)

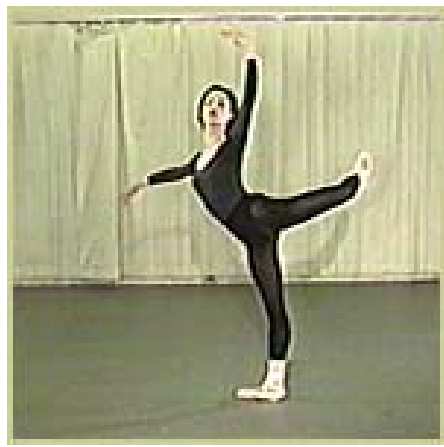

(b)

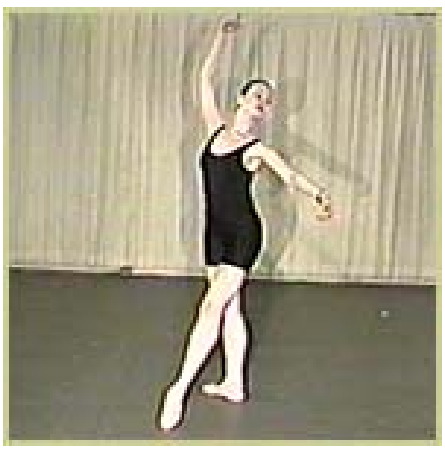

(c)

Figure 2.6: Three sample ballet poses: (a) Arabesque (b) Attitude and (c) Croisée. Images from the American Ballet Theatre's online ballet dictionary [55].

\subsection{Ballet Poses and Ballet Motions}

The approach proposed in this thesis is based on the classical ballet, because of the consistency of its vocabulary. This traditional style of ballet includes poses and motions that are well constrained. A ballet pose defines a relationship between hands, legs and head at a specific state of time. According to the American Ballet Theatre's dictionary [55] there are more than a hundred poses in ballet. Figure 2.6 shows three sample poses: Arabesque, Attitude and Croisée. Arabesque enforces putting the weight on one leg with the other leg extended to the back. Attitude is a pose in which the dancer stands on one leg while the other leg is lifted and well turned out with the knee bent at approximately 90-degrees. Croisée enforces to cross one leg in front of the other while facing toward a diagonal angle of 45-degrees.

A ballet motion is the set of in-between movements that connect two poses together. A combination of different motions blended together form a ballet sequence. In this work, to differentiate motions from sequences, they are referred to as minimotions. Figures 2.7 shows the Avant mini-motion which represents a forward move- 

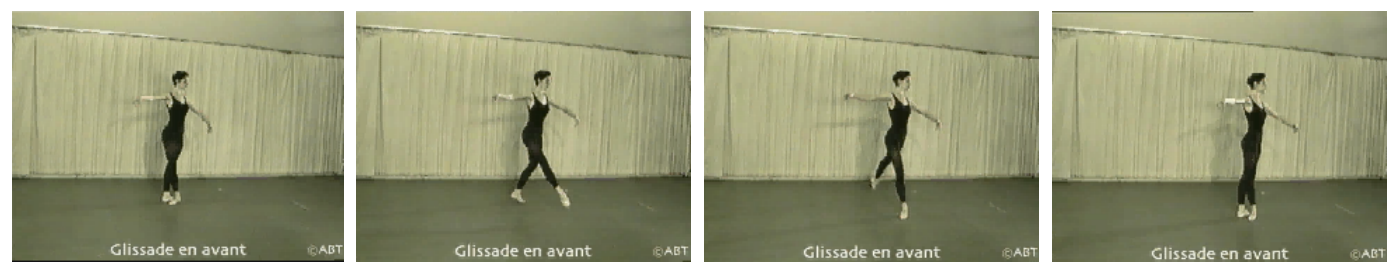

Figure 2.7: Four in-between screenshots of the Avant mini-motion. Images from the American Ballet Theatre's online ballet dictionary [55].
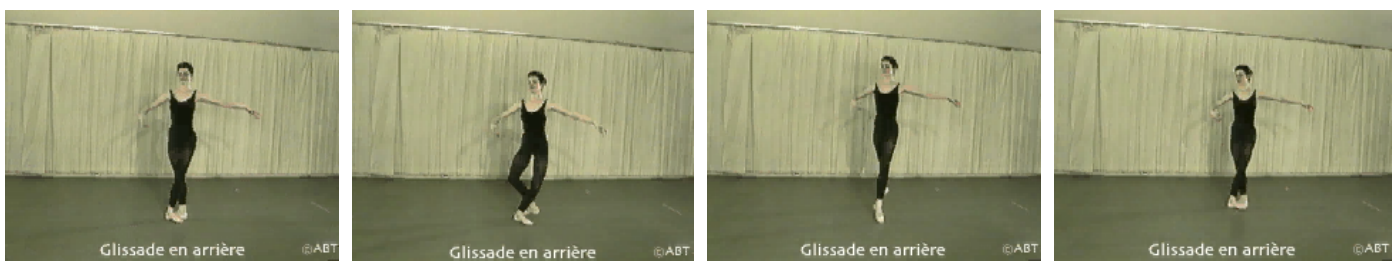

Figure 2.8: Four in-between screenshots of the Arriére mini-motion. Images from the American Ballet Theatre's online ballet dictionary [55].

ment toward the audience. Figure 2.8 shows Arriére mini-motion which represents a backward movement away from the audience.

\subsection{Ballet Master}

Since 1400s, the court dances became detailed and complicated, thus it was necessary to formally codify them to maintain their consistency. At this time, special dance instructors or "Ballet Masters" began to appear. They were highly respected and considered to be the finest dance teachers. During the early centuries of ballet companies (from the $18^{\text {th }}$ century until the early $20^{\text {th }}$ century), position of the First Ballet master was referred to the head of the company who acted as chief choreographer and artistic director. In modern times (after the early $20^{\text {th }}$ century), the title has been used more to describe master teachers or assistant directors of a ballet company (previously known as Second Ballet Master). Hence, the head of the ballet 

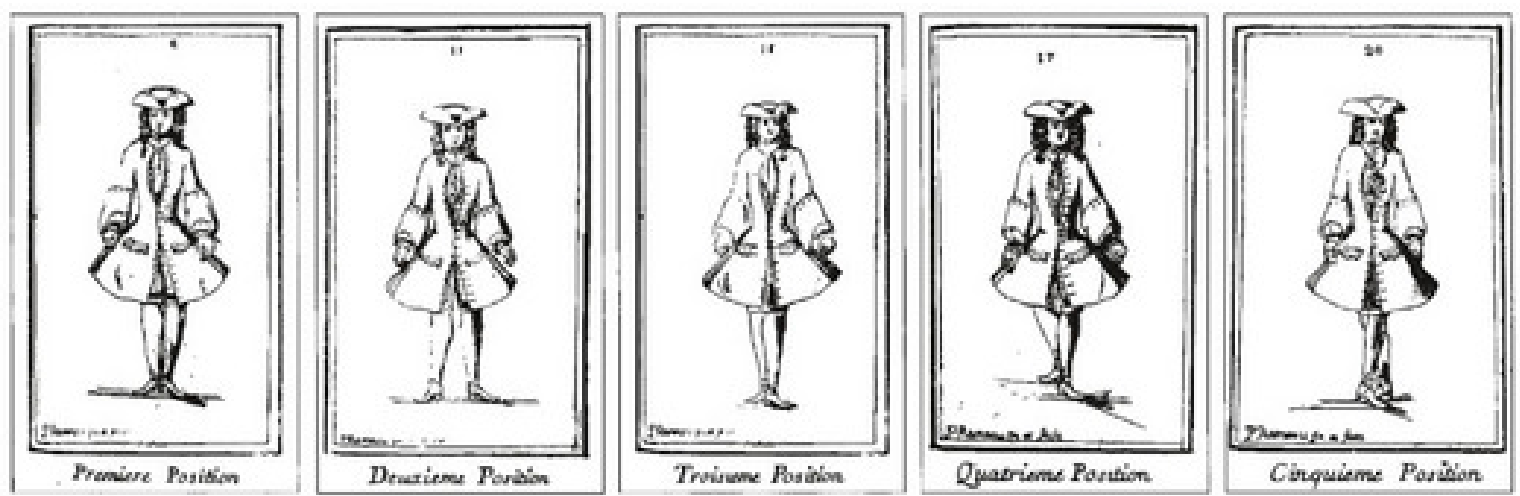

Figure 2.9: Pierre Beauchamps, a Ballet Master at the Royal Academy of Dance, is credited with developing the five basic foot positions of ballet. Image taken from [23].

company is only referred to as the artistic director.

Ballet masters are key employees of a ballet company because they are responsible for the level of competence of the dancers in the company. Traditionally, duties of the ballet masters include creating ballets, dances in operas, commissioning music, and managing the teaching of the dance classes. One of the earliest ballet masters at the Académie Royale de Danse was Pierre Beauchamp (1631-1705), who gave dance lessons to Louis XIV for over twenty-two years. Figure 2.9 shows five basic foot positions of ballet developed by Beauchamp. These positions are still the basis for all classical ballet steps, and are universally recognized even though their names are in the French language.

In modern times, ballet masters (or ballet mistress for a female) are generally charged with teaching the daily company ballet classes and rehearsing the dancers for ballet dance works. This research can help them better teach the dance steps to their students. 


\subsection{Basic Foot Positions}

Following, different foot positions in ballet are described in detail.

\section{Sole Position}

When the entire base of the foot touches the floor, it is in the sole position.

\section{First Position}

As shown in Figure 2.10, in the first position, the heels are brought firmly together, the points turned strongly outward and the knees held close together. This position is the one most commonly used in ballet.

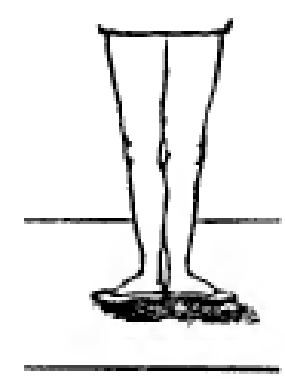

(a)

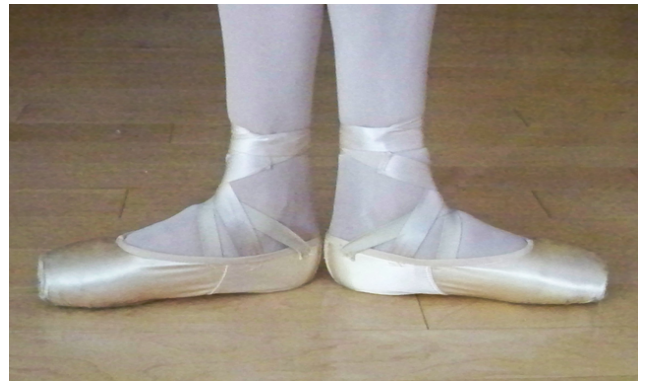

(b)

Figure 2.10: First position of the foot in ballet. Image (a) taken from [62]. Image (b) taken from Wikimedia Commons.

\section{Second Position}

As shown in Figure 2.11, in the $2^{\text {nd }}$ position, the foot is extended directly to the side as far as possible without raising the sole from the floor or transferring the centre of gravity. 


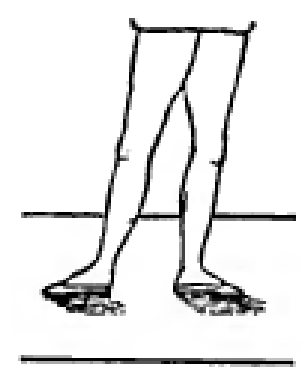

(a)

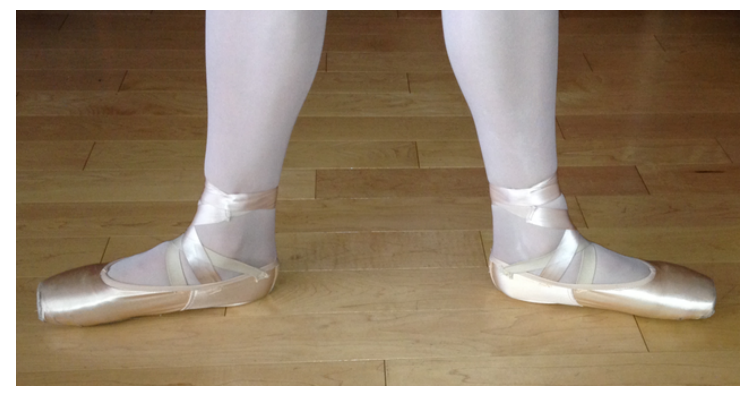

(b)

Figure 2.11: Second position of the foot in ballet. Image (a) taken from [62]. Image (b) taken from Wikimedia Commons.

\section{Third Position}

There are two phases of the $3^{r d}$ position. If for example, one places the right foot in $3^{\text {rd }}$ position forward (anterior) its heel touches the inner middle of the left as in Figure 2.12: but if the same foot be placed in $3^{\text {rd }}$ position behind (posterior), its inner middle (instep) touches the heel of the left foot.

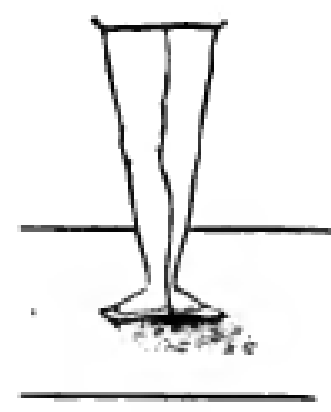

(a)

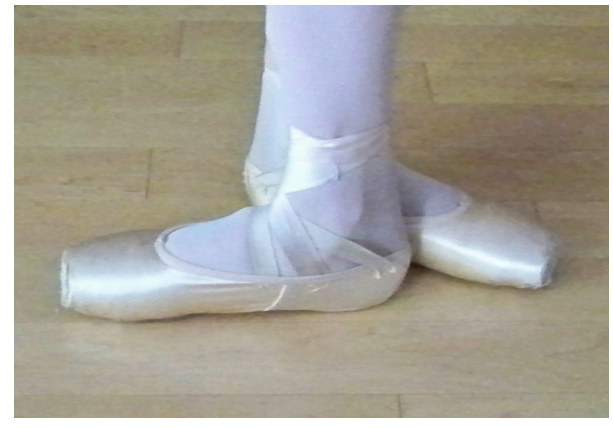

(b)

Figure 2.12: Third position of the foot in ballet. Image (a) taken from 62. Image (b) taken from Wikimedia Commons. 


\section{Fourth Position}

As shown in Figure 2.13(a), the $4^{\text {th }}$ position is attained by extending the foot in a straight line, either forward or backward, the length of one of the feet. Figure 2.13(b) shows two extra possibilities in each case: either heels are aligned (open) or the heel of the front foot is aligned with the toe of the back foot (closed).

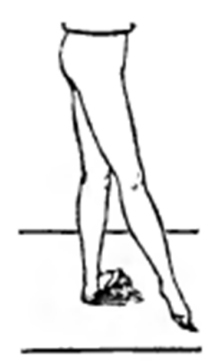

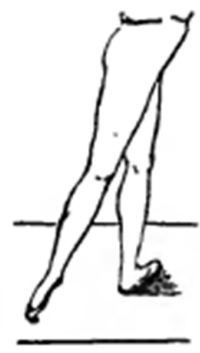

(a)
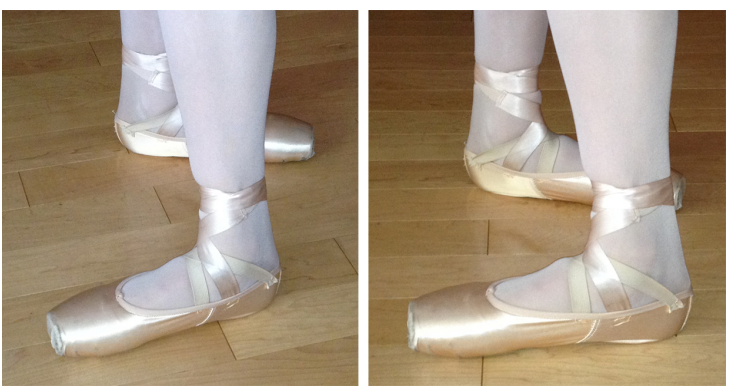

(b)

Figure 2.13: Fourth position of the foot in ballet. Image (a) taken from [62]. Image (b) taken from Wikimedia Commons.

\section{Fifth Position}

In the $5^{\text {th }}$ position, the legs are so strongly crossed that the heel of each foot touches the point of the other as shown in Figure 2.14.

\subsection{Choreography}

Choreography is the art of designing sequences of movements in which motion, form or both are specified. In dance, it mainly indicates designing and arranging dance movements. The word 'choreography' first appeared in the American English dictionary in the 1950s. It is derived from the Greek words for 'dance' and 'writing' which 


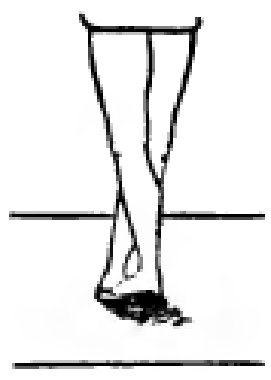

(a)

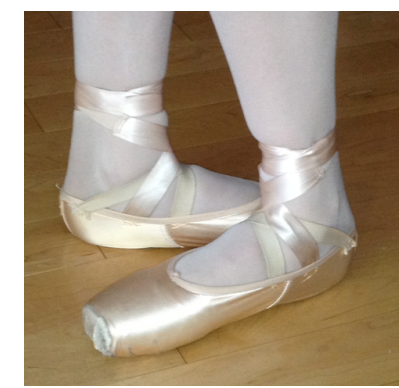

(b)

Figure 2.14: Fifth position of the foot in ballet. Image (a) taken from [62]. Image (b) taken from Wikimedia Commons.

literally means 'dance-writing'. In the $17^{\text {th }}$ and $18^{\text {th }}$ centuries, it was defined as "the written record of dances". In the $19^{t h}$ and $20^{t h}$ centuries, however, the meaning shifted inaccurately but universally, while the written record came to be known as dance annotation [12].

Choreography is used in a variety of fields other than dance, including: gymnastics, fashion shows, ice skating, marching band, show choir, synchronized swimming, animation and video game production. The art of choreography involves the specification of human movement and form in terms of space, shape and time. The choreographic process may also employ improvisation for the purpose of developing innovative movement ideas. On the other hand, planned choreography defines precise motions and forms with less room for personal interpretation. A movement language may also be used for this purpose, which is suitable for different dance techniques such as ballet.

During the history, dance choreography was mainly used to design dances that are intended to be performed in concerts. General aspects of the dance choreography include: the compositional use of organic unity, rhythmic or non-rhythmic articu- 
lation, theme and variation, and repetition. Traditionally, ballet masters served as dance makers or choreographers to create dances that can be taught to others. A choreographer is one who creates movements by practicing the art of choreography. The word 'choreographer' was first used as a credit for George Balanchine in the Broadway show "On Your Toes" in 1936 [54]. Prior to this, stage and movie credits were using phrases such as "ensembles staged by", "dances staged by" or simply "dances by" to denote the choreographer. Nowadays, choreographing the dance movements through writing record is known as dance annotation.

\subsection{Dance Annotation}

Dance annotation is a symbolic representation of the dance movement. It is parallel to the movement annotation but is narrowed down to represent specific forms in dance. The process of dance annotation requires reducing the four-dimensional movement of a dance (time being the fourth dimension) to a two-dimensional surface of paper [30]. Over the decades, various methods have been used to visually

represent dance movements. Guest [27] has gathered more than twenty annotation systems for ballet that can be divided into five categories:

- Word abbreviations

- Track drawings

- Stick figure (visual) systems

- Music note systems

- Abstract symbol systems. 
The aim of all annotation systems is to simplify the dance representation, however they are usually complicated to be understood by every one. The main challenge they have to address is to correctly convey the third dimension, which is missing in the two dimensional paper. The annotations that are based on word abbreviations or the trace of the foot or body of the performer on the floor, achieve simplicity but provide less 3D information. The music note annotations which are based on music staff, provide more information but are only useful for people who have a musical background. The abstract systems consider a rich language which must be learned. However, they do not look like movement annotations which makes it difficult to design an intuitive interface for them. The only easy to read annotation systems with good balance of information are stick figure systems. Compared to other annotation systems, they have fewer signs and use pictorial stick figure. Chapter 3 provides the history of these annotation systems and their analysis in detail.

\subsection{Motion Capture Technique (MoCap)}

One of the main challenges of this thesis was to create realistic digital motions with all the natural movements. Human motion has a lot of small details that should be considered in animation, since a small error can be noticeable. Motion Capture (MoCap) technique 25] addresses this issue by providing 3D animation clips with all the characteristics of a live performance. Therefore, it is used in the back-end of this work to achieve high quality dance motions.

To record motions in a Motion Capture setting, magnetic or optical markers are attached to the body of the performers. Then using special cameras the movements 


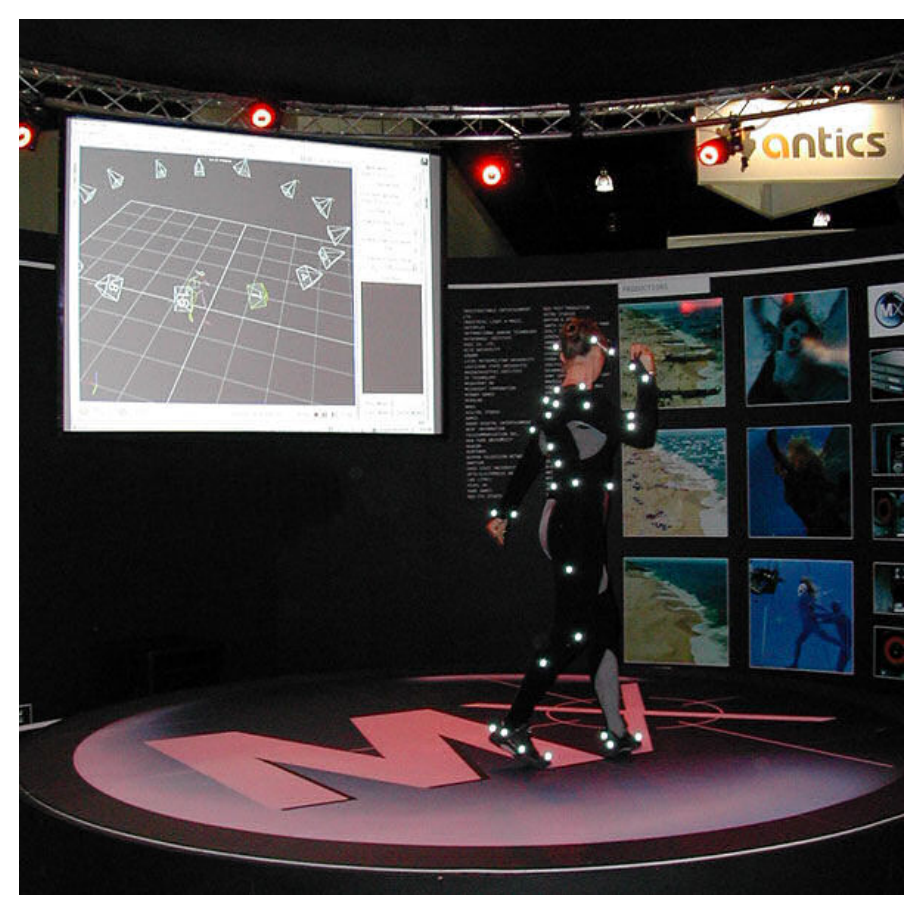

Figure 2.15: In motion capture, markers and special cameras are used to record a real movement. Image published under GNU License at Wikimedia Commons.

of these markers are logged. In this approach, only the movements of a character are recorded not its visual appearance (see Figure 2.15). A clip of motion gives a regular sampling of the character's parameters. These parameters include the positions of the root joint and quaternions representing the orientations of other joints.

The captured motion is usually imperfect and needs to be cleaned-up from unwanted artifacts such as footskate. It follows imperfections of a real performer and there is nothing but the data to describe the properties of the motion. Even when the MoCap perfectly captures the motion, there is still a need to edit the motion. It is not always possible to capture everything, since all movements cannot be preplanned. Some adaptation is necessary for 'impossible' situations that cannot be captured. Also motion needs to be edited for re-using on a different character. Some 
of these limitations are addressed by editing and adaptation of motion using signal processing techniques. Motion displacement mapping [13] provides a means to change the shape of a signal locally through a displacement map while maintaining continuity and preserving the global shape of the signal. Also, constraint-based motion adaptation [26] can be used to adapt previously created motion to meet new goals while retaining much of its original quality.

Human motion capture continues to be an increasingly active research area in computer graphics and computer vision. Recently, markerless video-based motion capture techniques [43] have opened a new realm to achieve photorealistic human motion. These techniques are no longer based on marker calibration and motion parameterization and just rely on the surface models and general shape deformation approaches. For example, De Aguiar et al. [20] extract motion constraints from video and make the laser-scan of the tracked subject mimic the recorded motion with a purely mesh based algorithm.

\subsection{Related Work}

Watching a performance and following the same steps is the typical means of learning a dance. Therefore, one might think that watching a captured video of a performance is enough for training. However, Guest [27] argues that filming a dance is not sufficient to communicate what is envisioned by a choreographer. Video-assisted systems

such as Dance Designer [17] (see Figure 1.5) enhance the learning process of dance but only support limited viewing angles. This is problematic as, during the recording of a dance, some movements may be obscured by the dancer from certain angles. 


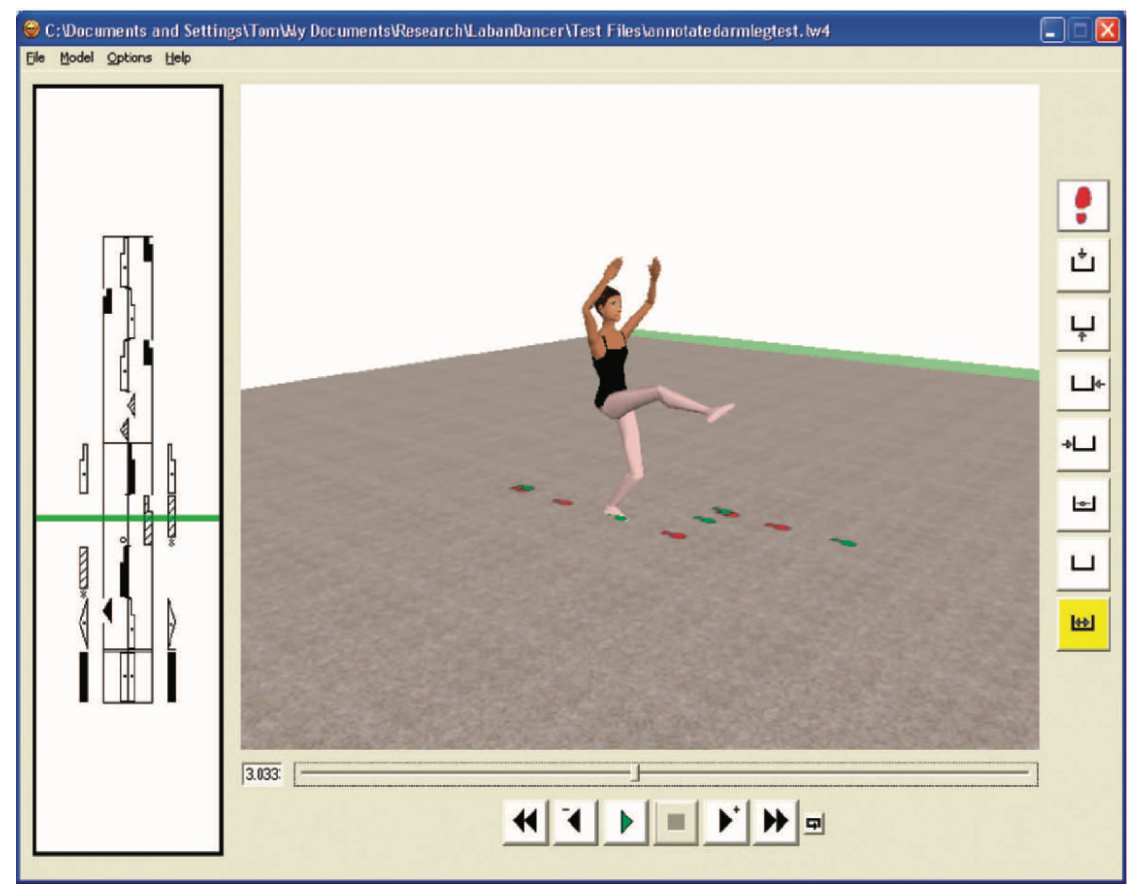

Figure 2.16: A user interface for representing the animation of a Labanotation score. Image taken from [15].

Also, in a large rehearsal it is not easy to capture all of the details of each dancer using videotape. James et al. [33] introduce a video-based choreography system that uses a visual stick figure interface to retrieve archival dance footages for synthesizing new videos. Their approach is useful when all videos are taken from a single viewpoint which is not enough to capture all of the spatial information. Also, videotaped dance may not show a perfect cast and it is not yet possible for choreographers to correct dance steps on the video.

DanceForms [15] tackles the lack of spatial information in 2D choreography by providing a 3D layer on top of an abstract notation [39]. As shown in Figure 2.16. it can visualize dance steps or entire routines in a 3D environment. While this helps in avoiding some of the ambiguities during dance composition and editing, the way 

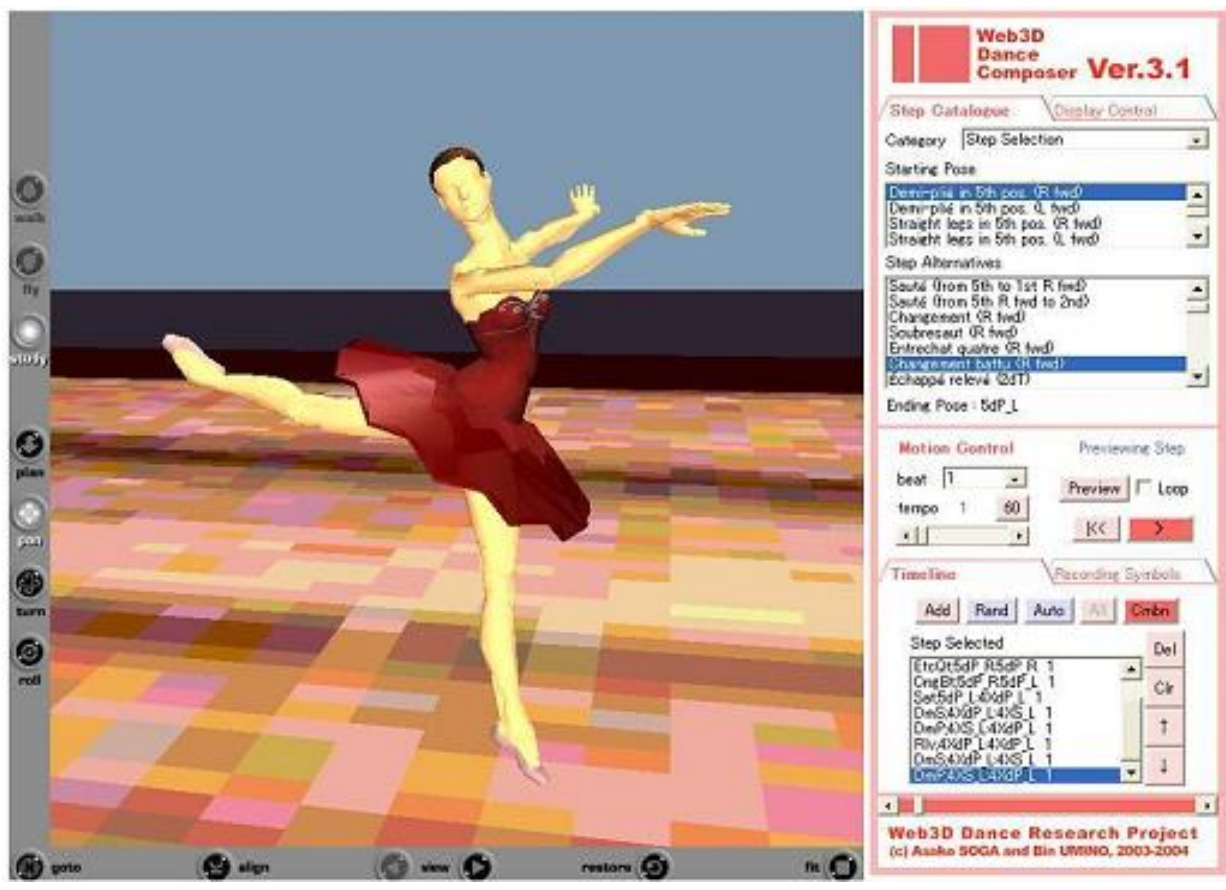

Figure 2.17: A screenshot of the Web3D Dance Composer. Image taken from [52].

it visualizes the dance steps is not very natural. In fact, the resulting 3D motions do not have all the details of the real performance. This results mainly from the limitations of DanceForms' dynamic animation generation.

As discussed in section 2.9, when creating new motions is not easy, one can use Motion Capture technique. Storing different motion clips in a MoCap database provides sufficient flexibility to represent high quality motions. Web3D Dance Composer [52] uses this technique to acquire the fundamental data from the performances of professional ballet dancers. Figure 2.17 shows a screenshot of the online interface provided by Web3D Dance Composer. As shown, it just provides a few selection lists to create the motion sequence which is not an intuitive way for freeform control of virtual dancer in the scene. It is demanding to have a sketch-based user interface for choreography that provides the same experience choreographers achieve when 
drawing and editing dance patterns on a piece of paper.

Sketch-based interfaces are growing rapidly because of their natural approach to interaction. They reduce the complexity of working with traditional interfaces by providing an intuitive way of communication. Instead of working with buttons, menus and dragging operations, people can now interact with drawings, editing and augmenting sketches. These electronic drawings can be parsed and converted to digital objects such as pictures, diagrams and 3D models [34]. Sketch-based modeling uses 2D sketches and 2D operations as an interface for 3D geometric modeling. Olsen et al. [48] have gathered a rich list of current sketch-based interfaces focused on 3D geometric modeling applications. This new metaphor is very helpful for novice users or experts in the early stages of design.

In recent years, sketch-based interfaces for doodling and controlling animation have gained popularity. Lee et al. [40] present the idea of real-time control of a three-dimensional character's motion in a 3D environment using a sketched path. They start from a set of motion clips and store them in a database. Then combine these motions in a two layer data structure. Higher layer is a statistical model that clusters similar data among motion clips and lower layer is a Markov process that creates new motions from a graph of motions. Motion graph [37] is a directed graph wherein edges contain either pieces of original motion clip or automatically generated transitions. The nodes then serve as choice points where the small bits of motion join seamlessly (see Figure 2.18). Motion graphs transform the motion synthesis problem into one of selecting sequences of nodes, or graph walks. This technique provides realistic, controllable motion, however, it requires that initial members of each cluster be picked by hand. Therefore, the application designer should know the 


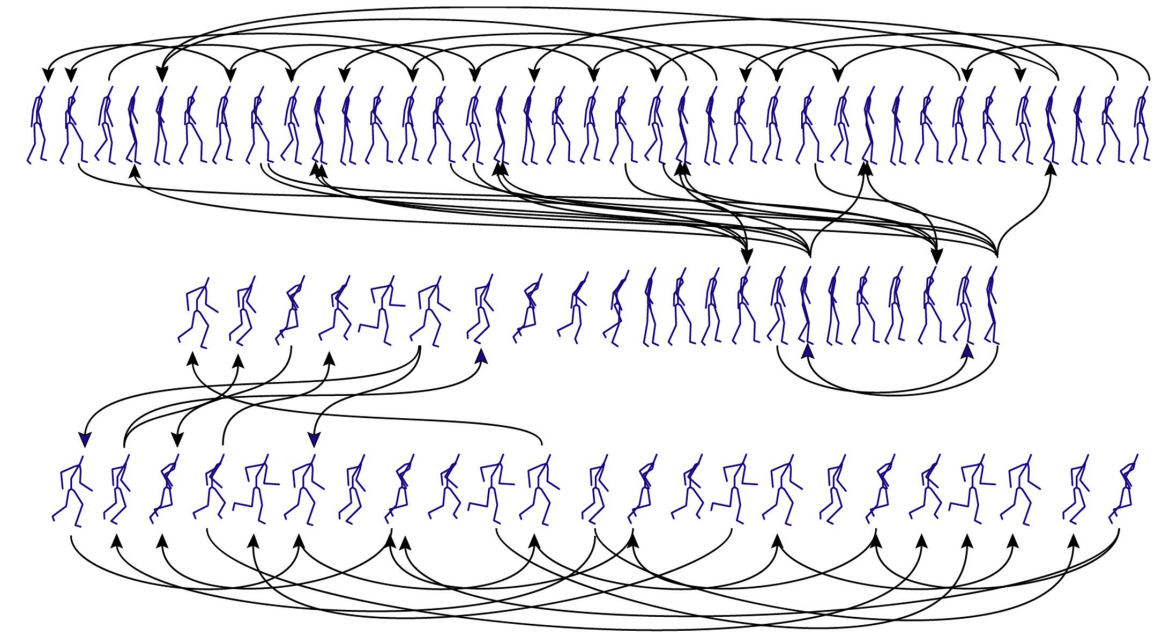

Figure 2.18: An illustration of a Motion Graph. Image taken from [37].

motion database sufficiently well to select widely dispersed poses as cluster seeds.

Davis et al. [19] introduce a new sketching interface to rapidly create articulated $3 \mathrm{D}$ animations from $2 \mathrm{D}$ sketches of the character in the desired key frame poses. As illustrated in Figure 2.19, one can draw different skeletons of the character as 2D stick figures, and system constructs the sequence of 3D poses that exactly match the input drawings. One can also guide the system to the desired character pose, using appropriate constraints, to fine tune the resulting animation. Thorne et al. [56] use a different sketch-based approach for sketching a character and its motion. User begins sketching a character by drawing links for the head, torso, upper arm, lower arm, upper leg, lower leg, and foot. After drawing the figure, the system automatically determines the skeleton for the character and creates a 3D model. Then by doodling a motion gesture in a 3D environment for character, it starts moving along that path (see Figure 1.3). In fact, the user inputs are parsed and mapped to a parameterized set of output motions to reflect the location and timing of the input sketches.

Oshita [49] presents a pen-based intuitive interface to control human motion in- 


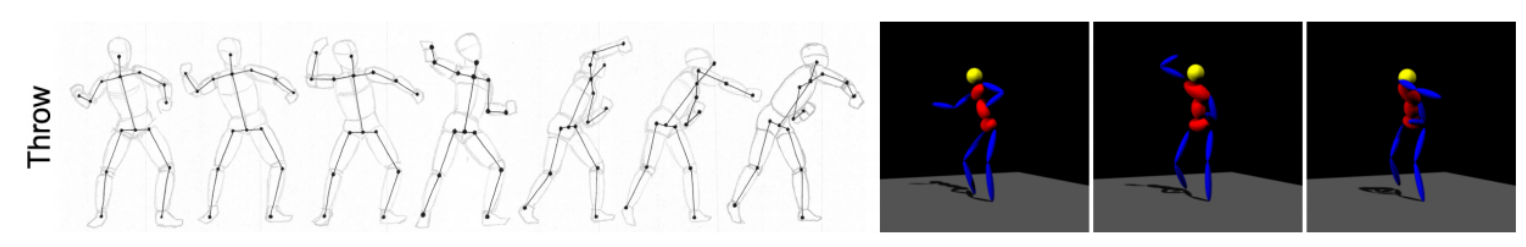

Figure 2.19: Drawn keyframes of a throw motion and the final 3D representation. Image adapted from [19].

teractively. He employs the pen as a metaphor of sketch, and directly maps pen's position, pressure and tilt to the motions of a 3D avatar. Figueroa et al. [24] introduce a sketch-based interface for creating animation with sound from a drawing on paper. They use a combination of object symbols, actions and arrows for animation generation. MotionMaster [41] provides a sketch-based approach for choreographing Kungfu motions at the early stage of animation creation (see Figure 2.20). User first draws a 2D mannequin figure and label its joints. Then draws a line on this figure to show the trajectory of the moving joints. If the $2 \mathrm{D}$ posture and the trajectory are valid, the candidate motion will be retrieved from the motion database. Next, the user can edit the 2D sketches and the changes will be directly applied to the 3D motion sequence. In this way, the approximated motions can be iteratively optimized and refined by user to be used later in the production. These techniques are useful for generating expressive motion in real-time; however, they support a limited subset of gesture vocabulary in 3D such as direction, orientation and speed, which is not enough to create detailed dance motions.

Regardless of all the efforts in the digital choreography systems and the advances of sketch-based systems for controlling animation, the intersection of these two is mostly untapped. Figure 2.21 summarizes the specifications of the major related works to this research and indicates the satisfaction degree of each criterion based 


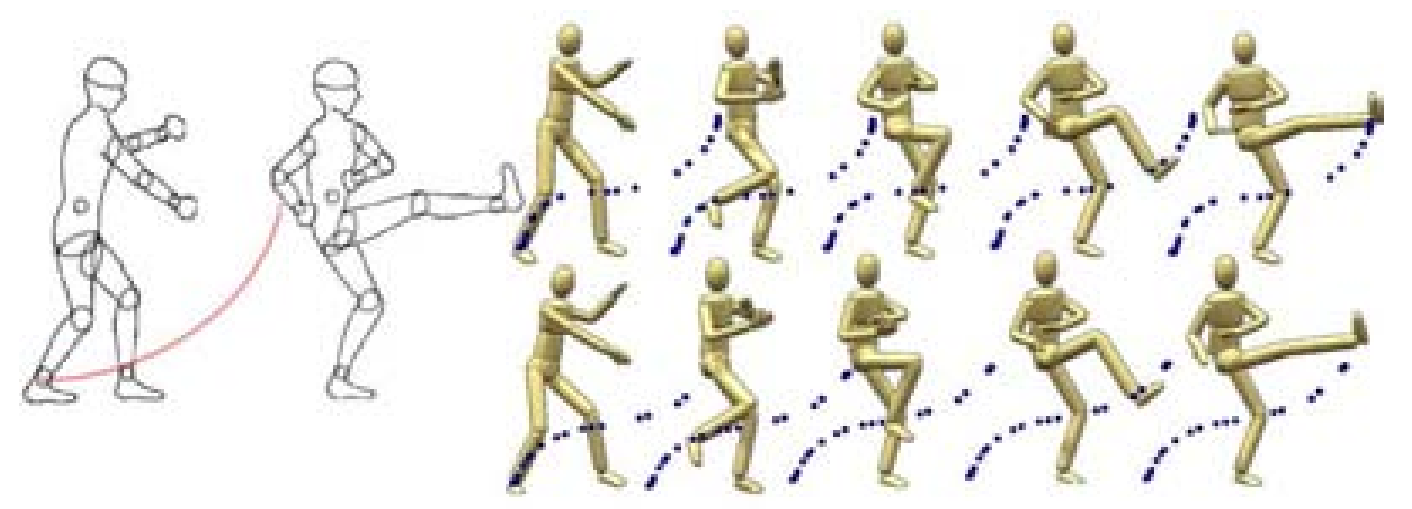

Figure 2.20: An illustration of the MotionMaster. Image adapted from [41].

on a five point scale (Poor, Fair, Average, Good and Excellent). These specifications are in line with the main objectives described in chapter 1.2. They provide useful criteria for the design and evaluation processes of the proposed approach.

\begin{tabular}{|c|c|c|c|c|c|c|c|}
\hline & Motion Capture & Sketch Based & Video Based & 3D Environment & 2D Environment & Annotation & Menu and Keyboard Based \\
\hline DanceForms [15] & & & & Poor & Good & Good & Excellent \\
\hline $\begin{array}{l}\text { Web3D Dance } \\
\text { Composer [51] }\end{array}$ & Good & & & Average & & & Fair \\
\hline $\begin{array}{l}\text { Dance Designer } \\
\text { (Choreopro) [17] }\end{array}$ & & & Good & & Fair & & Good \\
\hline Lee et al. [40] & Excellent & Good & & Excellent & & & \\
\hline Motion Master [41] & & Good & & Fair & Average & & \\
\hline James et al. [33] & & Average & Excellent & & Poor & & \\
\hline
\end{tabular}

Figure 2.21: Comparison of the major related works to this research based on their features. 


\section{Chapter 3}

\section{Annotation Analysis}

The symbolic representation of dance movements is called dance annotation [27]. Annotations are crucial part of the dance choreography and should be chosen carefully. In order to create a sketch-based system for choreography, a simple and comprehensive annotation system should be used. The preference of this work is to employ annotations that can be understood by novice users. Also, it is vital that there should not be any ambiguity when a 3D motion is created out of these annotations. This chapter analyses the most important annotations used in dance choreography to find a good candidate suitable for this research.

\subsection{Dance Annotation}

Dance annotation is a recording of the dance movement through the use of written symbols and 2D drawings. The process of dance annotation translates the fourdimensional movement (time being the fourth dimension) into signs written on twodimensional paper [30]. A fifth dimension for dynamics, or the quality and texture of the movements should also be considered as an integral part of the annotation, however in most cases it is not. There are more than twenty annotation systems for ballet choreography [29]. Figure 3.1 lists some of these notations which are divided into five categories. Among them, only the most relevant systems to this work will be analyzed. 


\begin{tabular}{|l|lllll|}
\hline Words Abbreviations & Cervera & Arbeau & Playford & Meunier & Saunders \\
\hline Track drawings & ${ }^{\circ}$ Feuillet & Lorin & Landrin & \\
\hline Stick Figure (visual) & Saint-Leon & ${ }^{\circ}$ Zorn & ${ }^{\circ}$ Benesh & Sutton \\
\hline Music Note & ${ }^{\circ}$ Stepanov & Conte & Nikolais & \\
\hline Abstract Symbol & Theleur & ${ }^{\circ}$ Laban & Morris & Loring ${ }^{\circ}$ Eshkol-Wachmann \\
\hline
\end{tabular}

Figure 3.1: Ballet dance notation systems.

\subsection{Words and Word Abbreviation Systems}

The most trivial annotation is to use a letter for the name of each step in the dance.

In this way, dance could be represented by listing each initial letter of the steps in the sequence. The first printed book using this letter coding technique was "LÁrt et instruction de bien dancer" published in France in the late $15^{\text {th }}$ century [50]. As shown in Figure 3.2, each letter is placed under appropriate music note as a kind of memory aid. Many ballet dancers have written these notes by themselves to memorize the steps. However, it may be difficult for another dancer to be able to recreate the same pattern. Therefore, more words and more details are required which necessitates better organization and standardization. 


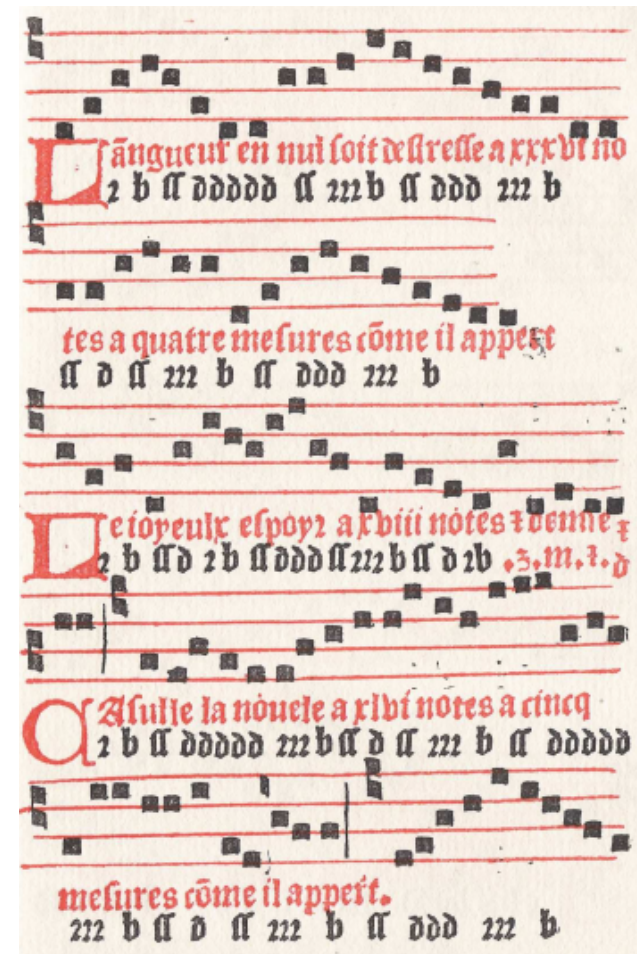

Figure 3.2: An example of word abbreviation notation in LÁrt et instruction de bien dancer. Image taken from [50].

\subsection{Track Drawing Systems}

The first known attempt to gather a full set of dance annotations was a book published in Paris at 1700 by Raoul-Auger Feuillet [23]. In his book, entitled "Chorégraphie ou lárt de décrire la danse" (Choreography, or the Art of Describing the Dance), Feuillet introduces a notation which is based on adding specific symbols to the trace of the dancer on the floor. Figure 3.3 shows an example choreography using this notation. It shows the music score for the section of the dance at the top of the page. The annotation shows a couple starting at the back of the room, moving forward, then separating to move on quarter-circle arcs away from each other before taking a straight path to meet again. 


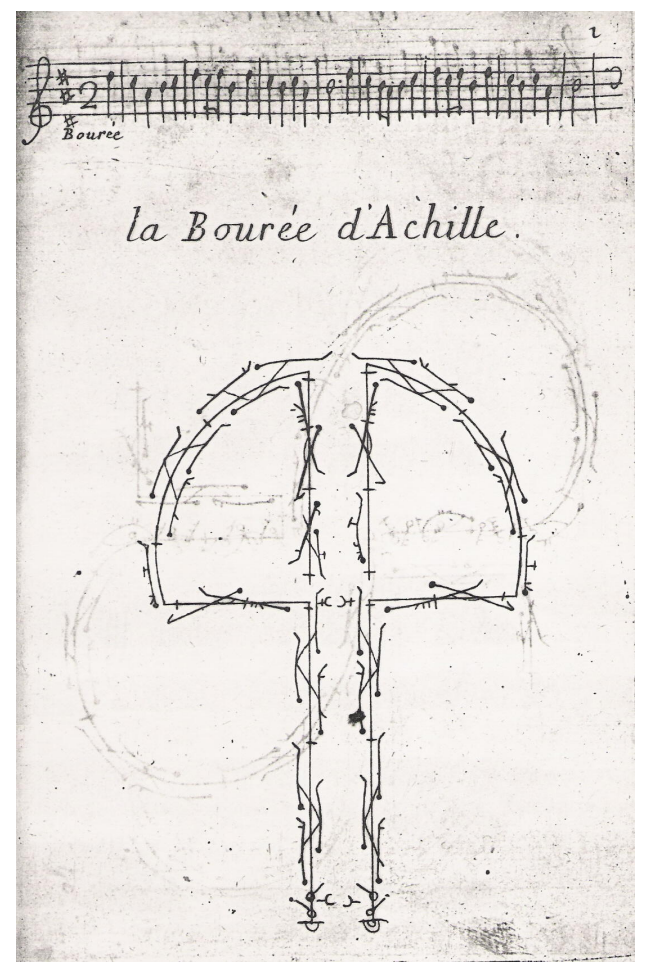

Figure 3.3: An example of Feuillet annotation based on track drawing for The Bourée dÁchille. Image from [23] available through Britanica.

In order to better understand the annotations used in Figure 3.3 representations of foot position, arm position, jump sequence, walk sequence and turn sequence in Feuillet annotation system are provided in sections 5.3.1, 5.3.2, 5.3.3, 5.3.4 and 5.3.5 respectively. The sketch-based approach introduced in this research, is partly inspired by Feuillet annotation system, because it provides a visual clue as to the location of the dancer in the scene.

\subsection{Stick Figure (Visual) Systems}

After Feuillet, many other annotations were provided by choreographers. Some of these annotations come under the heading of visual or pictorial systems, because of 


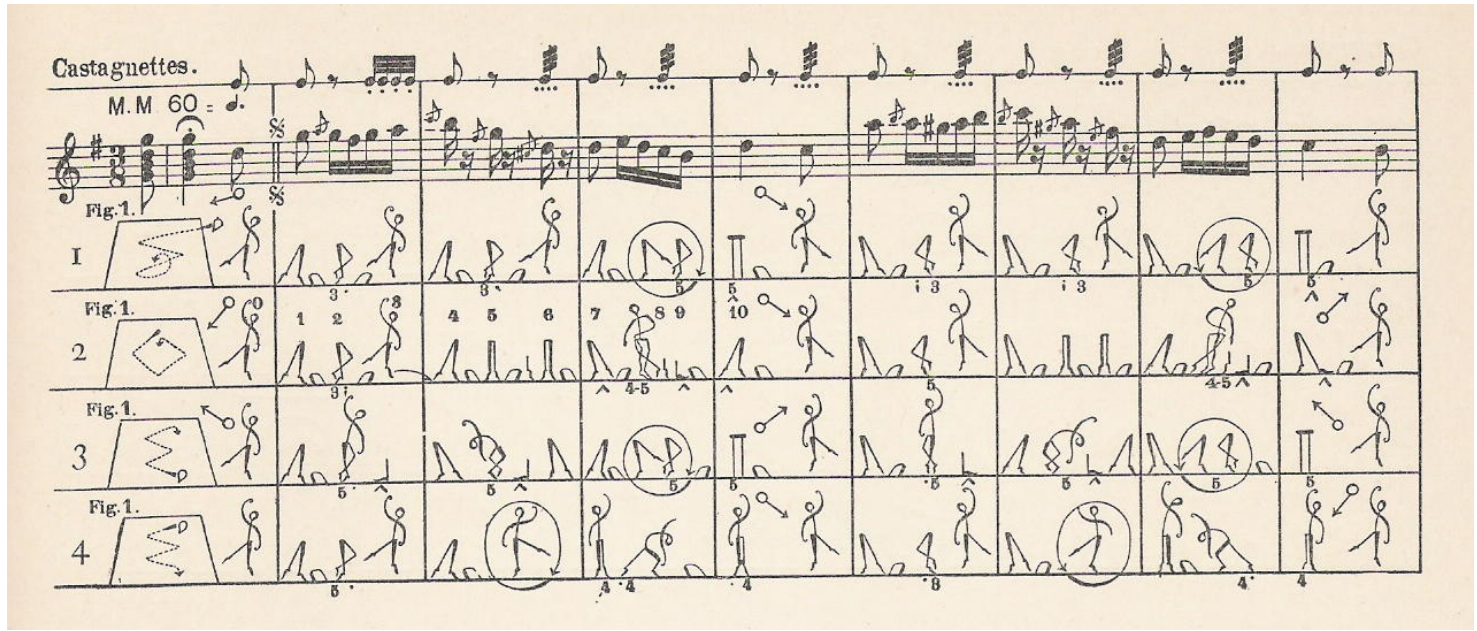

Figure 3.4: Extracts from the score of Fanny Elssler's Cachucha choreographed using Zorn's system. Image from [62] available through Wikipedia.

the obvious use of stick figures, while others use more stylized drawings. The stick figure systems emerged in the middle of the $19^{\text {th }}$ century in France and Ukraine. The first choreography system based on stick figures was called "Stenochoreography" [51] which was published by Arthur Saint-Léon in Paris in 1852. Friedrich Albert Zorn [62], a successful German dancer, choreographer, and dance theorist, extended this notation in Ukraine and published his book about the "Grammar of the Art of Dancing" in 1905. Figure 3.4 represents a sample choreography using Zorn's annotation system. Zorn uses only one staff line as an indicator of the base floor, because the stick figure itself shows the pose of the body. Hence, it only needs to indicate when the body is on the floor or in the air. Often only the lower part of the body is indicated when concentration is on the leg work.

Later, in 1940, Rudolph Benesh [10] provided Benesh movement notation, which is still used in many companies including Royal English Ballet. Benesh's system uses five horizontal staves, similar to the staves of a musical score (see Figure 3.5). 


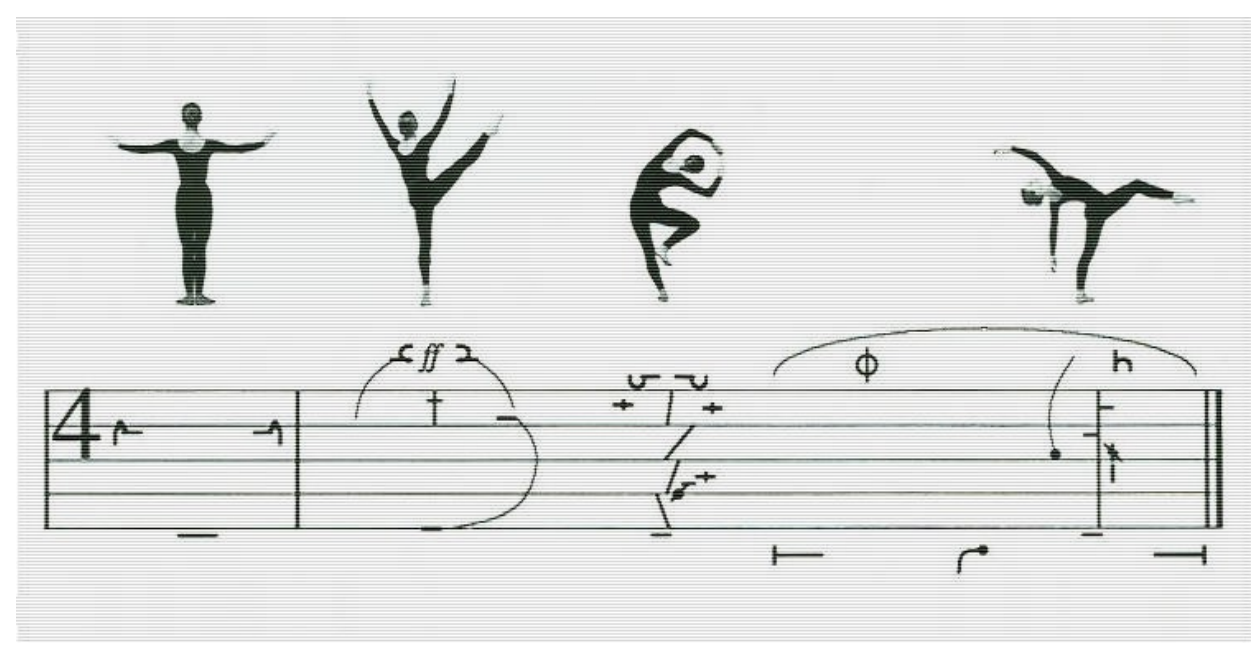

Figure 3.5: An example of recorded movements in Benesh movement notation. Image taken from Royal Academy of Dance website [45].

These lines are read from left to right, where each line refers to one part of the body. Figure 3.5 shows a sample of recording movements using Benesh movement notation. Because of using abstract symbols in this system, it is less intuitive to work with compared to the other stick figure systems.

In order to better understand the annotations used in Figures 3.4 and 3.5 representations of foot position, arm position, jump sequence, walk sequence and turn sequence in Zorn and Benesh annotation systems are provided in sections 5.3.1, 5.3.2, 5.3.3, 5.3.4 and 5.3.5 respectively. The representational feature of the stick figure annotations provides an immediately understandable message. On the other hand, the more abstract annotations require more learning. Zorn's system is easy to read and it has comparatively few additional signs. The simplicity of Zorn's annotation inspired this work to use stick figure-based annotations for sketch-based dance choreography. 


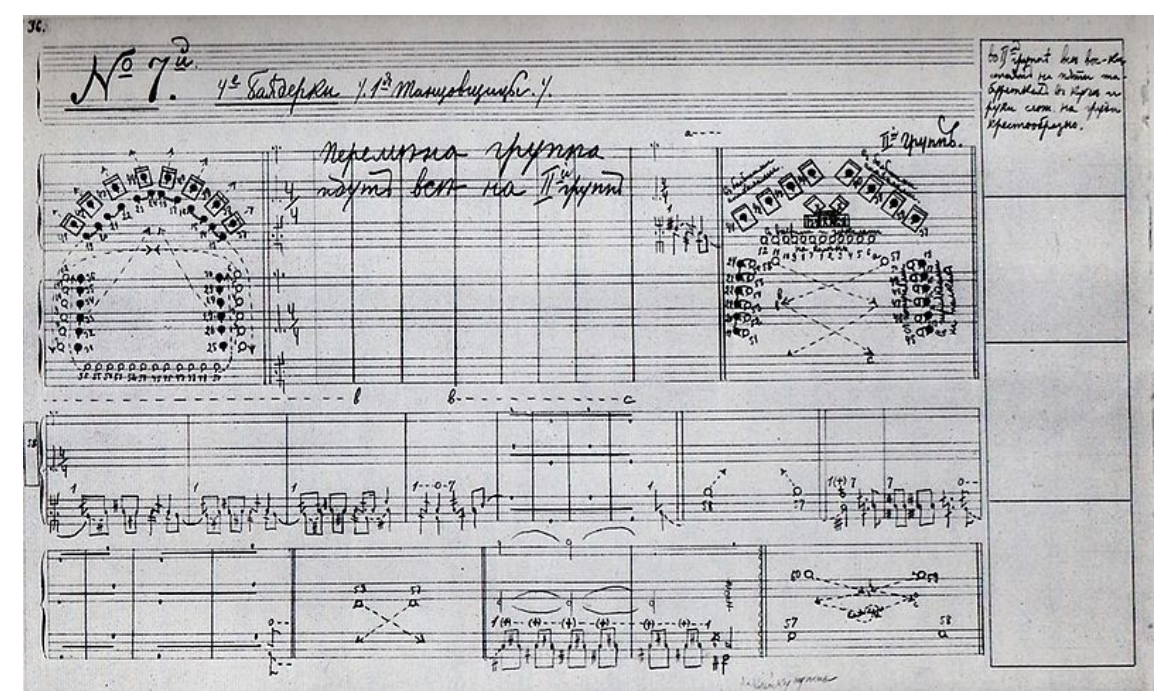

Figure 3.6: A sample choreography using Stepanov's notation. Image available in the public domain through Wikimedia commons.

\subsection{Music Note Systems}

Another notation for ballet was developed by Vladimir Stepanov [53] that was published in his 1892 book entitled "Alphabet of Movements of the Human Body". As shown in Figure 3.6, his notation mostly focuses on the adaptation of motions to music notes. In most annotations, a stave is used to represent the body and its parts. In music note systems the stave is derived directly from the horizontal music staves. Stepanov divides music stave into three sections: top for the head, center for the arms and bottom for the legs. The lines and spaces on the staves are used to show either direction or the specific part of the body which is moving.

Some people who have a musical background object to mapping movement annotations to music notes. They believe such usage is confusing rather than helpful, particularly to a young student who prefers non-musical signs for recording dance. In addition, these annotations make it difficult to design a suitable sketch-based input 


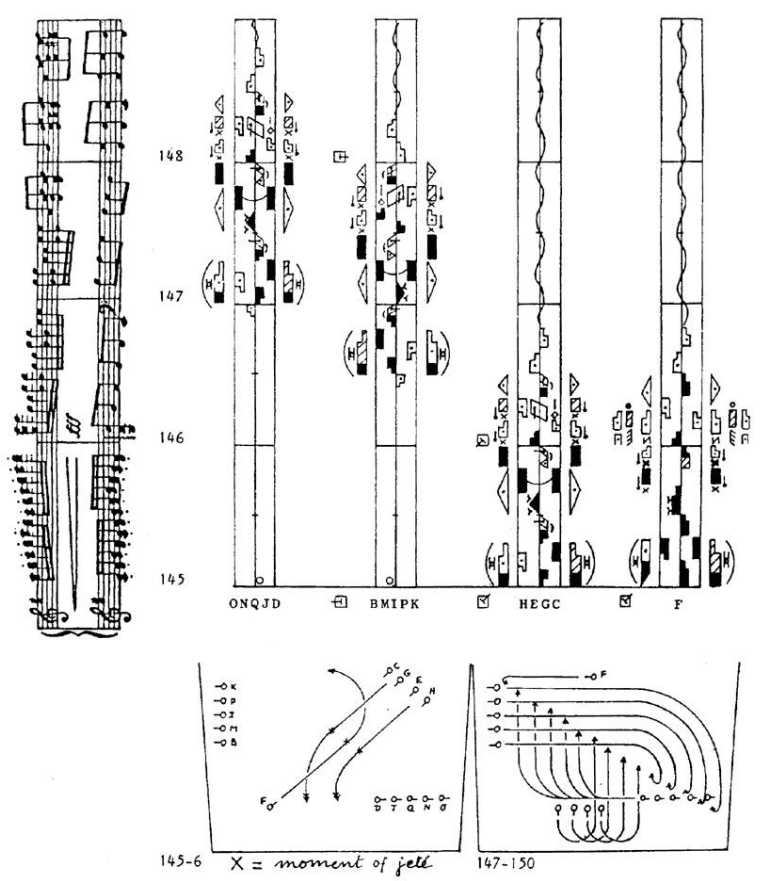

Figure 3.7: Laban's notation system. Image taken from [39].

mechanism for dance choreography compared to stick figure systems.

\subsection{Abstract Symbol Systems}

In the 1920s, Rudolf von Laban [39] introduced a system, known as labanotation, for analyzing and recording human movements. As shown in Figure 3.7, to represent the body position, his system uses three staves that run vertically from bottom to the top. Laban was inspired from the Feuillet system in his design and used the center line to divide the body into right and left. However, his vertical staff has seemed to many people to be an odd and unnecessary innovation since both music notation and verbal writing progress horizontally across the page.

More recently (in 1958), Noa Eshkol and Abraham Watchmann [22] introduced 


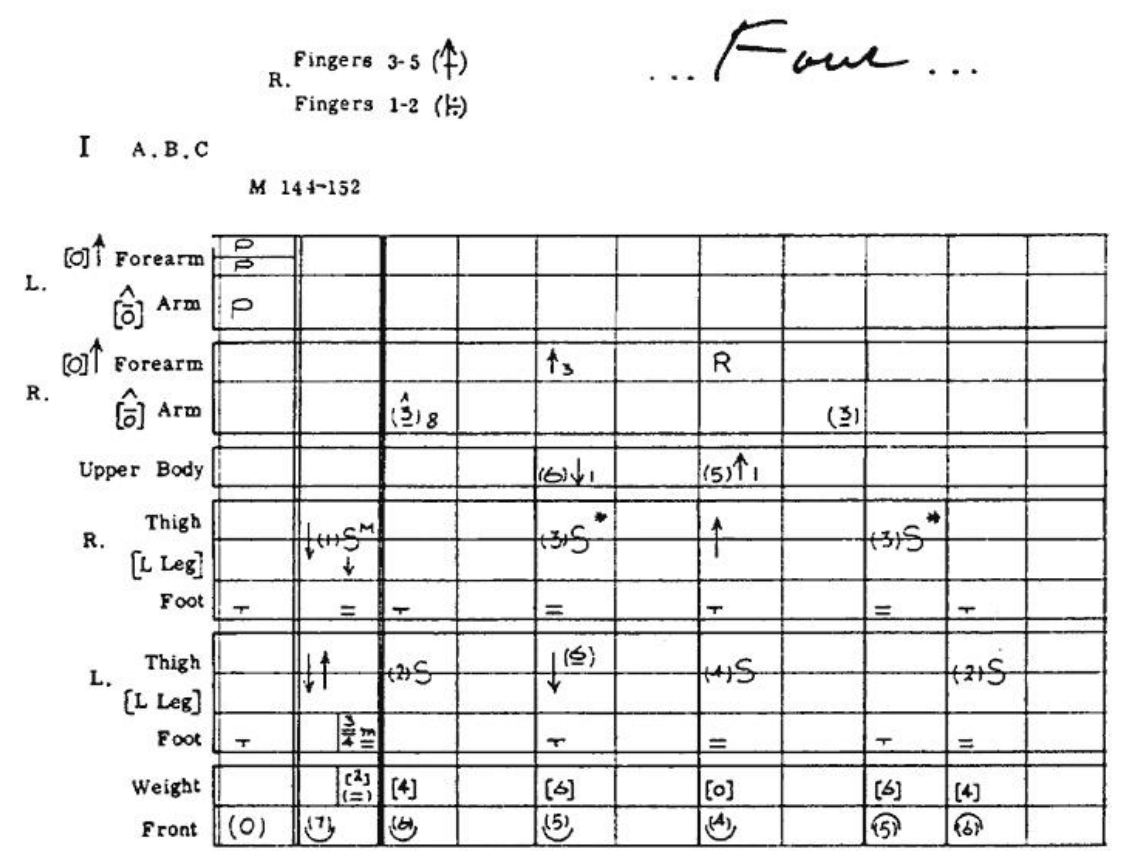

Figure 3.8: An example of the dance notation system developed by Noa Eshkol and Abraham Wachmann. Image available in the public domain through Wikimedia commons.

a new movement notation called Eshkol-Wachman. They use numbers and small set of symbols to record each possible movement. As shown in Figure 3.8, they provide a complete horizontal staff with spaces for each body segment and individual signs for parts of the body.

\subsection{Analysis}

A common problem of the dance annotation systems is that few choreographers and even fewer dancers are literate in them. Writing a dance score needs time and practice, as does writing a book or a symphony. However, Guest [29] reveals that often the time spent learning to read and write annotations is considered as wasted. 
The field study done for this project indicates that using the current annotation systems for choreography makes it impossible for a choreographer to compose a dance work in the studio. The choreographer is limited by the relatively short period of time allowed for rehearsals. Therefore, it is difficult to make changes to the annotations and experiment in the same way a music composer can. As a result, each choreographer uses her own style for composing dances which usually can only be interpreted by herself. This explains why the current dance annotation systems are mostly used for the recording of dances in the studios, rather than creating and learning, of dances.

Guest [29] believes that, regardless of the workable systems that have been around for centuries, the use of dance annotation has never been an integral part of dance study and practice, as musical notation is in the study of music. An important reason for this limitation in the traditional choreography techniques is that they just rely on 2D annotations for composing a 3D dance movement. In traditional painting and drawing, this lack of dimensionality does not prevent them to communicate in 3D. However, in dance this may cause communication ambiguities between choreographers and dancers. During the past decade, evolution of sketch-based techniques has provided some suggestions for addressing dimensionality limitations in 3D interfaces and modelling. However, there is a little effort on creating sketch-based solutions for dance choreography.

To provide a suitable annotation system for the proposed approach, the pros and cons of the current annotations have been evaluated. First, the major tools that are now used in choreography as well as their underlying annotations (if any) were examined (see Figure 2.21). Then, the most popular annotation systems currently 
used in ballet community were investigated. Well-known dance academies were the center of focus for this part. Finally, a few domain experts were debriefed to find out their level of familiarity and opinions about these annotation systems. The result of this evaluation suggests that annotations which use the trace of the foot or body of the performer on the floor e.g. [23] aid in understanding the dance better. However, they project the $3 \mathrm{D}$ movements onto a $2 \mathrm{D}$ plane, which is not enough to represent all spatial information. On the other hand, the music note annotations e.g. [53] are helpful for another important aspect of dance. However, they can add the complexity of the input annotations. Also, regardless of the richness of the abstract systems e.g. [39, 22] they are difficult to be used as a simple doodling/sketching interface in compare with the stick figure systems [62, 10] that are easy to read and have less ambiguity. Therefore, it seems that stick figure systems are a better choice for this research. 


\section{Chapter 4}

\section{Methodology}

To achieve the research goals highlighted in chapter 1.2 a user-centered methodology has been pursued. In this methodology, the main requirements of the end users have been the center of the attention at each stage of the project. First, a few ballet masters and choreographers have been informally debriefed to arrive at their main needs. Then, the related literature has been reviewed to gather the main specifications that satisfy these needs (see chapter 2). Next, the history of the annotation systems has been researched (see chapter 3) to evaluate the pros and cons of each annotation. The last step is to aggregate the field studies and the specifications found during literature review to propose a novel solution for the research question.

This chapter provides a detailed description of the proposed sketch-based approach to dance choreography. First, the field study done for this project is explained. Next, the design process undertaken for this work is described. Then, the new stick figure annotation system developed for this project is introduced. Next, the process of preparing and acquiring motion capture clips for each annotation, or mini-motions, is discussed. Then, the sketch recognition approach that assigns new annotations to these mini-motions is explained. Finally, the synthesizing mechanism which blends the recognized mini-motions, i.e. a sequence of keyframes into a dance animation is described. 


\section{$4.1 \quad$ Field Study}

The "user-centered design" (UCD) methodology in HCI suggests to focus on the end user requirements. To be specific, Norman [46] offers four basic suggestions on how a design should be:

- Make it easy to determine what actions are possible at any moment

- Make things visible, including the conceptual model of the system, the alternative actions, and the results of actions

- Make it easy to evaluate the current state of the system

- Follow natural mappings between intentions and the required actions; between actions and the resulting effect; and between the information that is visible and the interpretation of the system state.

The core recommendation of the UCD is to place the user at the center of the attention. Therefore, at the beginning of the project, current challenges of choreography have been explored to provide a suitable solution. In this regard, some informal interviews were conducted with a few ballet masters, choreographers and university instructors. During these interviews, many notes were taken about the current process for choreography in a studio and what can be done to improve this process. One of the choreographers mentioned that he usually takes video from his choreography and then uploads it to a private group on Vimeo. Dancers then watch this video and memorize the movements by rehearsing them at their own pace. Also, one of the ballet masters indicated that her role is to memorize every movement in the sequence in case the choreographer forgets them. 
Another technique that was used by one of the dance instructors was doodling the poses on a white board. This dance instructor records her initial choreography by drawing a few stick figures on a white board. Then, progressively fine-tunes this initial design during rehearsal until every mistake is corrected. It was interesting that the annotations used by this instructor for recording the movements were similar to the Zorn annotation system [62].

These interviews showed that most of the choreographers are not interested in using advanced dance annotation systems. Some of them use video to record the dance while many of them prefer to record the dance using simple stick figures because they find it more aligned with their artistic direction in dance. This motivated developing a simplified version of stick figure annotation systems for this research. To sum up, the combination of the field study and the literature review on the current tools (see section 2.10 helped to arrive at these specifications for the proposed approach:

- Using motion capture technique

- Have an sketch-based interface

- Provide a 3D environment for choreography

- Support a 2D environment for choreography

- Use simplified stick figure annotations.

There is no specific hardware requirement for development of this project and any off the shelf desktop or laptop machine with standard specifications would work. However, it is recommended to use a tablet and pen for evaluation of this technique in studios because of its closeness to the pen and paper metaphor. 

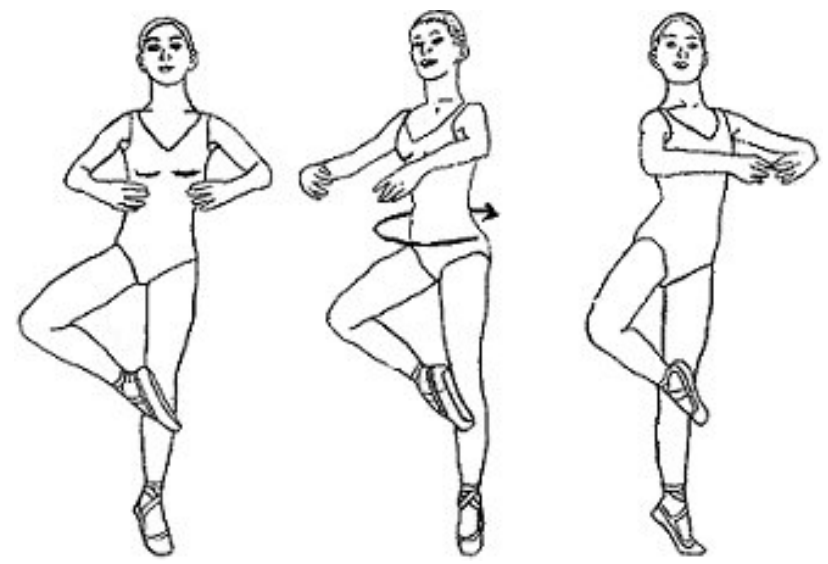

Figure 4.1: Pirouette mini-motion. Image taken from [47].
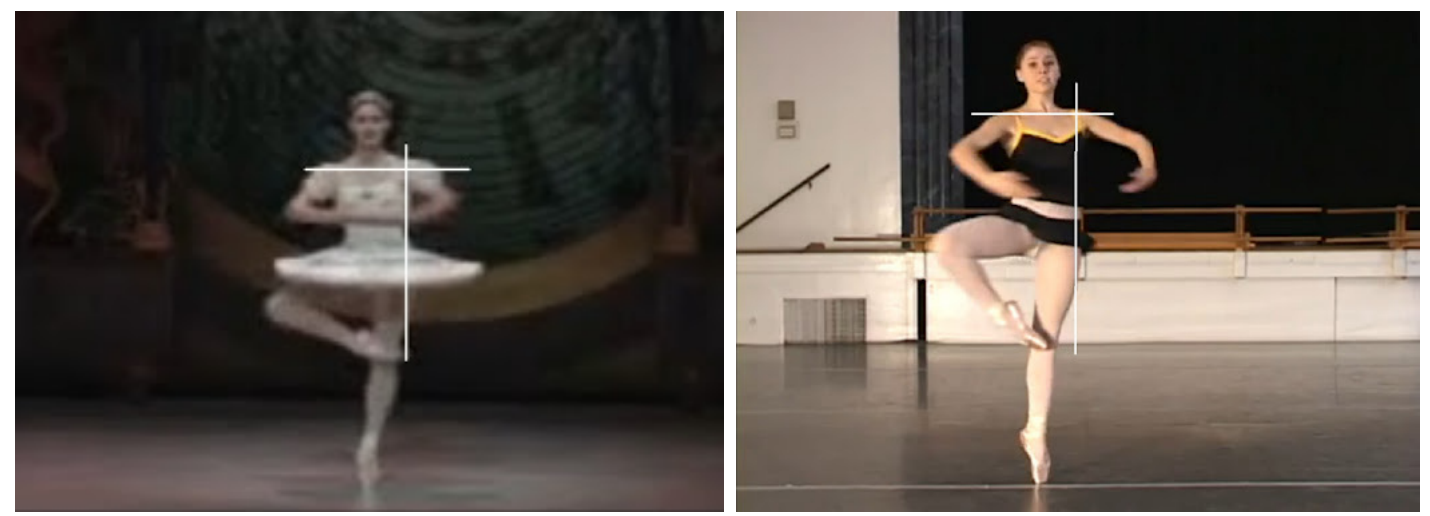

Figure 4.2: Pirouette mini-motion played by two different dancers. Images taken from [14].

\subsection{Design Process}

Classical dances such as ballet usually consist of limited and constrained motions. For example, Figure 4.1 represents the Pirouette mini-motion which is a complete turn of the body on one foot. As shown in Figure 4.2, both dancers playing Pirouette follow the same steps. This example shows that, ballet movements are strongly constrained. This is the main reason that ballet is chosen for the proposed choreography approach.

As discussed in section 4.1, 'user-centered design' methodology suggests to put 
user at the center of the design. The design process of this project follows seven principles introduced by Norman [46]:

- Use both knowledge in the world and knowledge in the head. By building conceptual models, write manuals that are easily understood and that are written before the design is implemented.

- Simplify the structure of tasks. Make sure not to overload the short-term memory, or the long term memory of the user. On average the user is able to remember five things at a time. Make sure the task is consistent and provide mental aids for easy retrieval of information from long-term memory. Make sure the user has control over the task.

- Make things visible: bridge the gulfs of execution and evaluation. The user should be able to figure out the use of an object by seeing the right buttons or devices for executing an operation.

- Get the mappings right. One way to make things understandable is to use graphics.

- Exploit the power of constraints, in order to give the user the feel that there is one thing to do.

- Design for error. Plan for any possible error that can be made, this way the user will be allowed the option of recovery from any possible error made.

- When all else fails, standardize. Create an international standard if something cannot be designed without arbitrary mappings. 


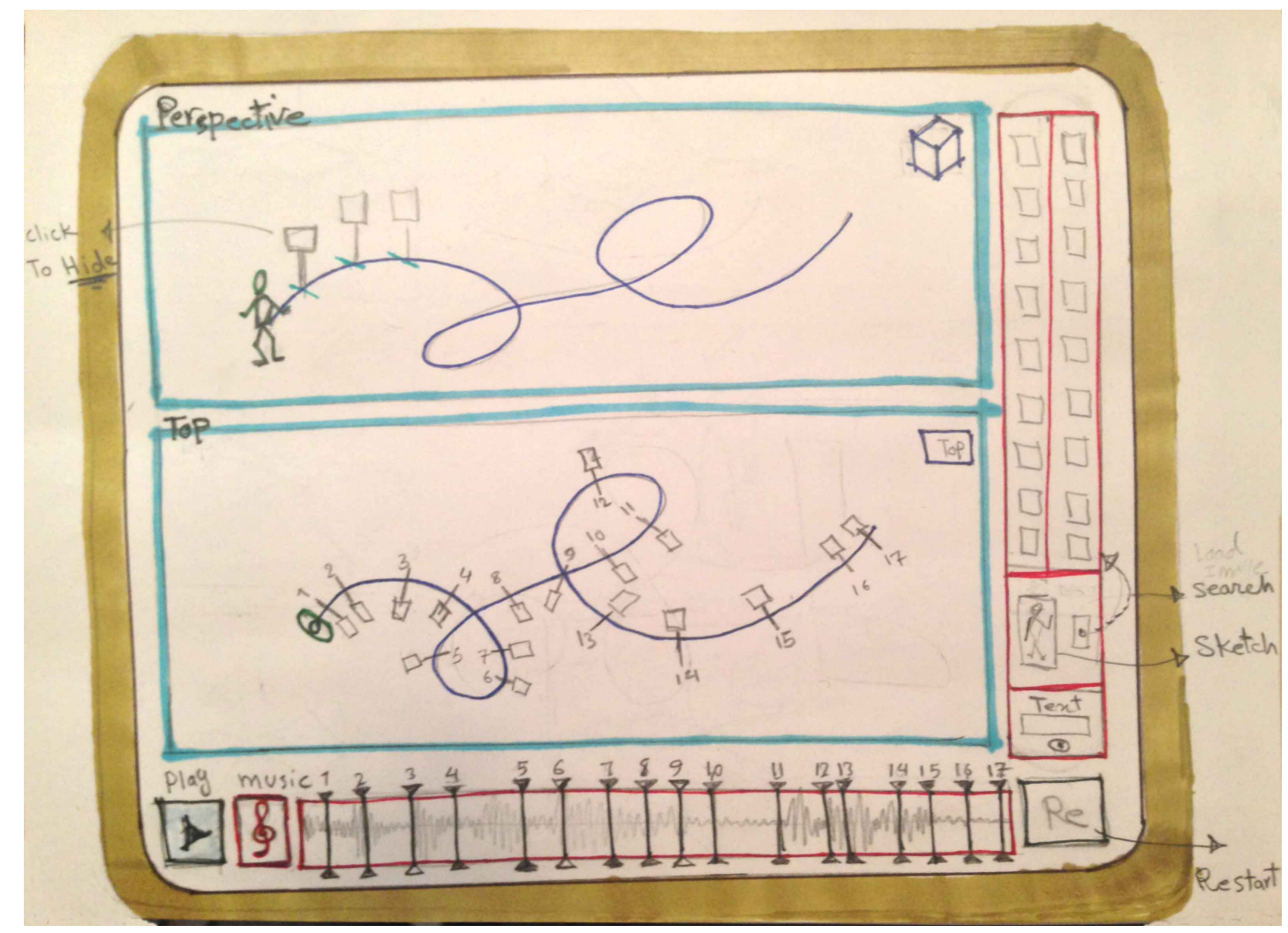

Figure 4.3: An early concept for the user interface of the proposed approach.

In order to comply with the user-centered design principals and the field study discussed in section 4.1, the design process of this project is centered around drawing stick-figure annotations. Figure 4.3 shows an early concept designed for the proposed approach. It represents the trajectory of the dancer drawn as a single stroke in perspective view and top view (similar to the track drawing systems). In addition, it allows drawing a stick-figure (inspired from the stick-figure annotation systems) in the bottom-right corner to retrieve corresponding mini-motions. To allow choreographers doodle a dance sequence intuitively, a simple set of stick figure based annotations should be created for representation of mini-motions. A 3D animation clip i.e. mini-motion should then be assigned to each of these sketched annotations. 


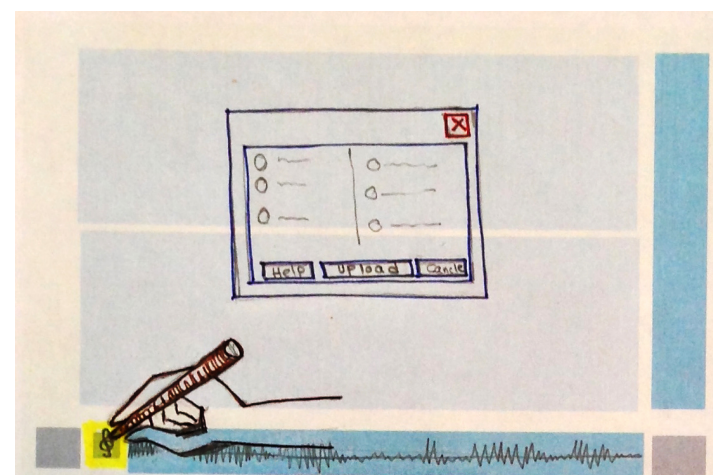

1. upload a music

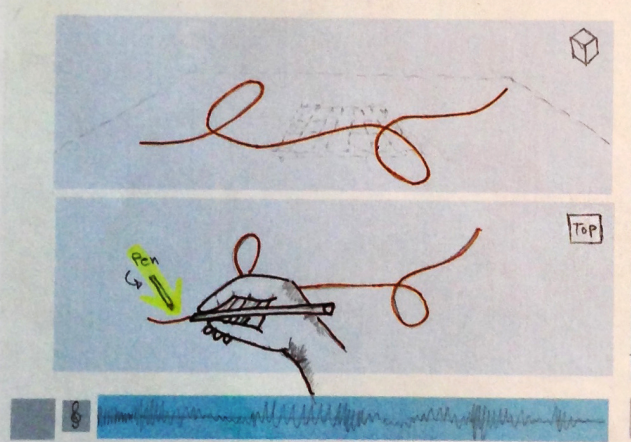

3. Draw a trajectory

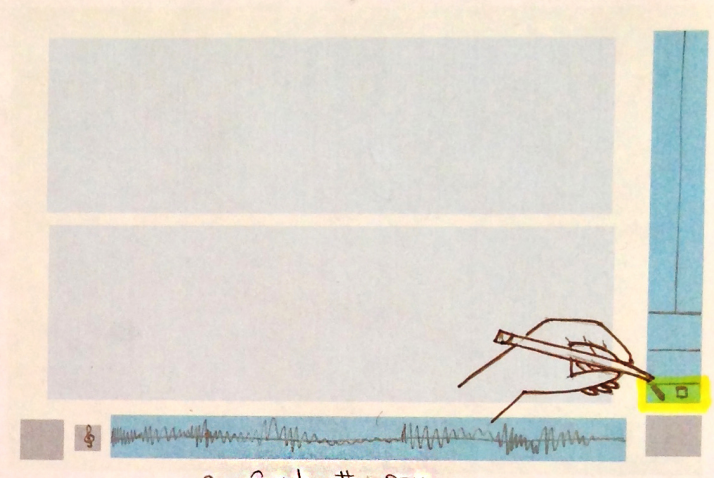

2. Grab the pen

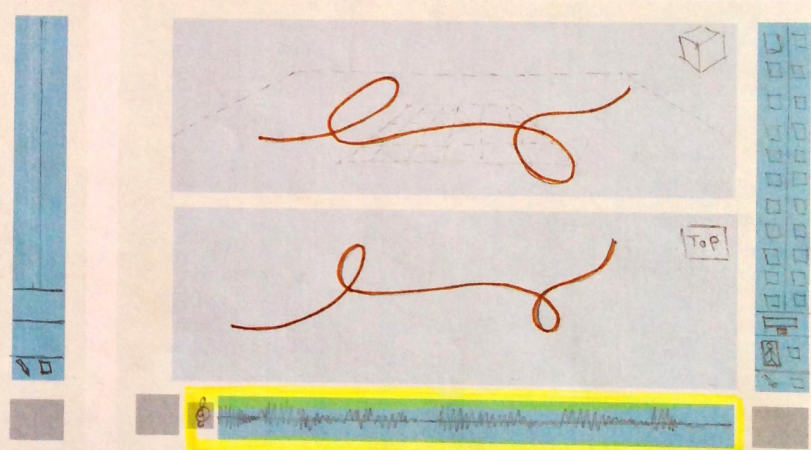

4. Listen to the music
and think about movement

Figure 4.4: A sample storyboard for sketch-based dance choreography.

Figures 4.4, 4.5 and 4.6 illustrate the designed storyboards for combining 3D motions along the sketched trajectory to form a dance sequence. The process starts by selecting a music score for the sequence at step 1 . Then the trajectory of the dancer is drawn at step 3. A text search is envisioned at step 5 for finding the minimotions by entering their name. Also at step 6 , a stick figure can be drawn to find the closest match in the database of poses. Then at step 8 the chosen mini-motion will be placed on the trajectory. This process can be repeated until all mini-motions of the sequence become complete. Finally, by pressing the play button at step 12 the mini-motions will be blended together and create the final sequence. 


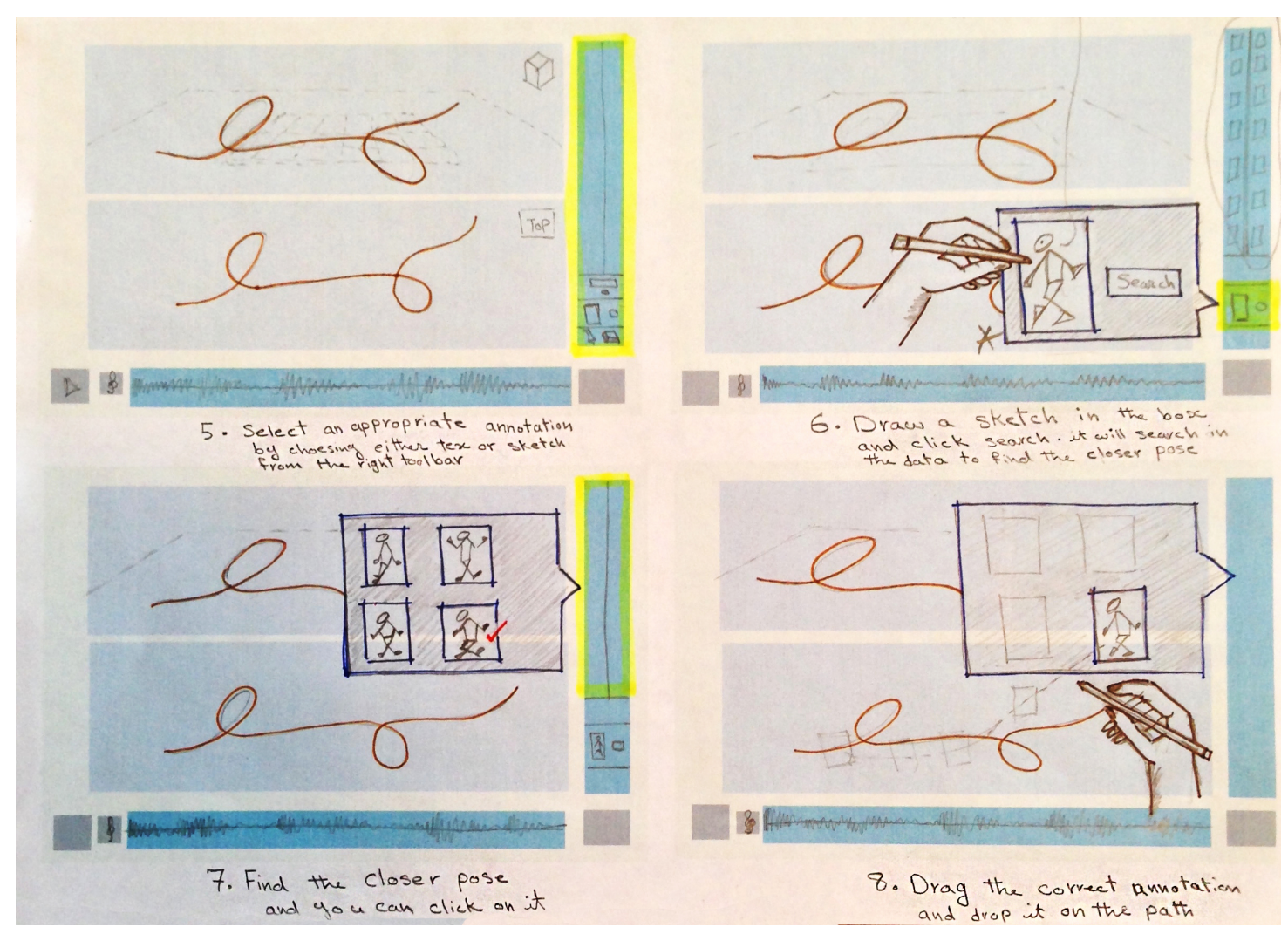

Figure 4.5: A sample storyboard for sketch-based dance choreography.

The initial design for the proposed approach evolved over the course of the project. For example, a billboard was originally envisioned for each mini-motion to represent its position on the trajectory. However, it was removed from the design to avoid clutter. Instead, mini-motions were assigned to a movement list to be edited easily. Editing has a critical role in choreography, because it allows removing, replacing and changing the order of the movements in a sequence. Figure 4.7 summarizes the core concepts of the proposed design for creating a dance sequence from a set of mini-motions. In this pipeline, the efficiency of the $2 \mathrm{D}$ process is maximized by providing a 3D animation of the dance. This can help choreographers design, edit and communicate different dance patterns with all of their details. 


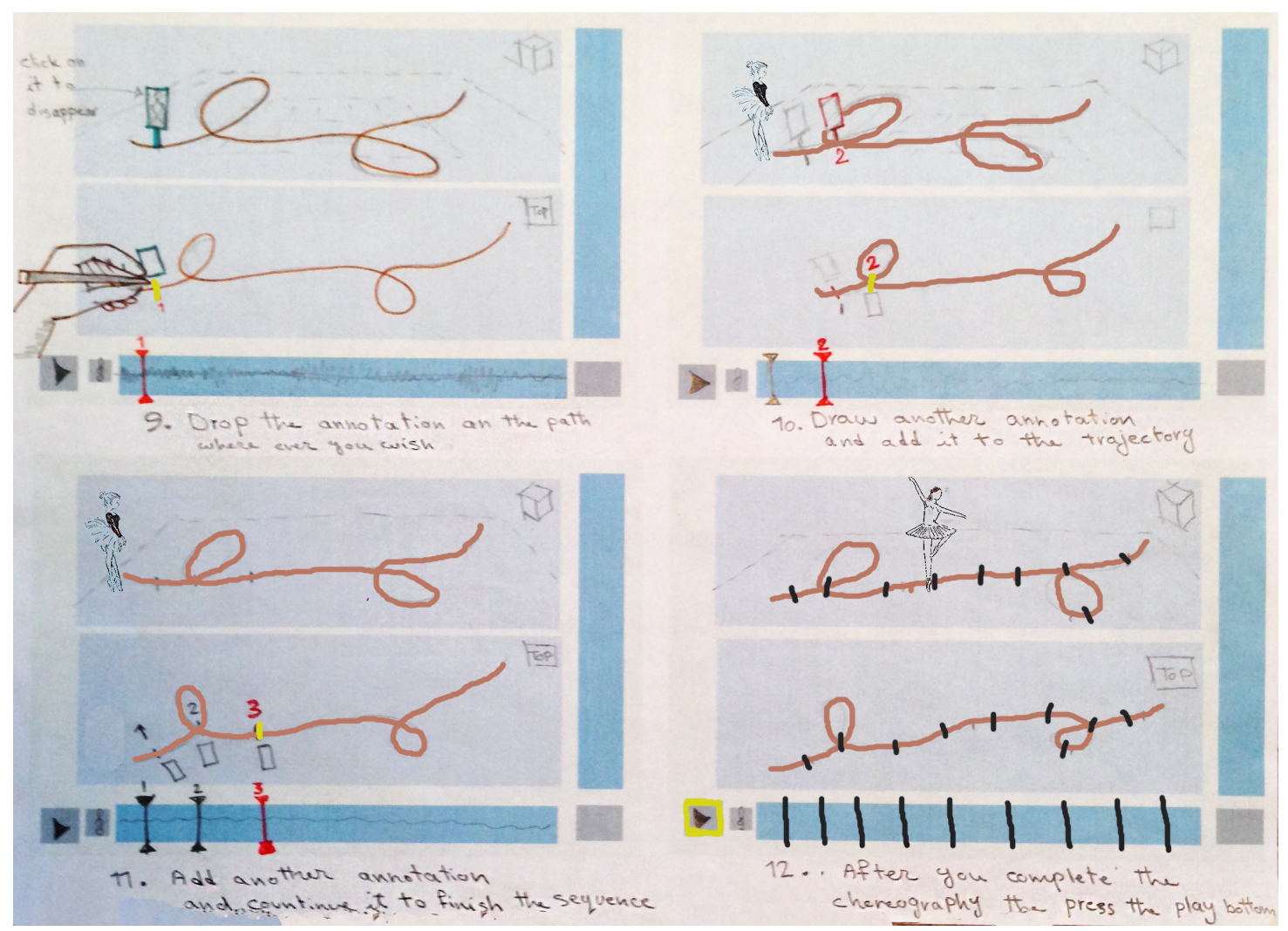

Figure 4.6: A sample storyboard for sketch-based dance choreography.

\subsection{Stick Figure Annotation}

An important stage in the proposed approach is finding an easy to follow yet comprehensive annotation system to represent 3D dance movements. The field study described in section 4.1 highlighted the tendency of choreographers toward simpler annotations, preferring stick figure annotations over abstract symbols. This motivated a research on finding simplified versions of the common stick figure annotations. The outcomes of this research proposed a new annotation system which is inspired from Zorn's annotation system [62]. The new annotations do not follow the full range of Zorn's detailed annotations, as only one annotation is needed to retrieve 


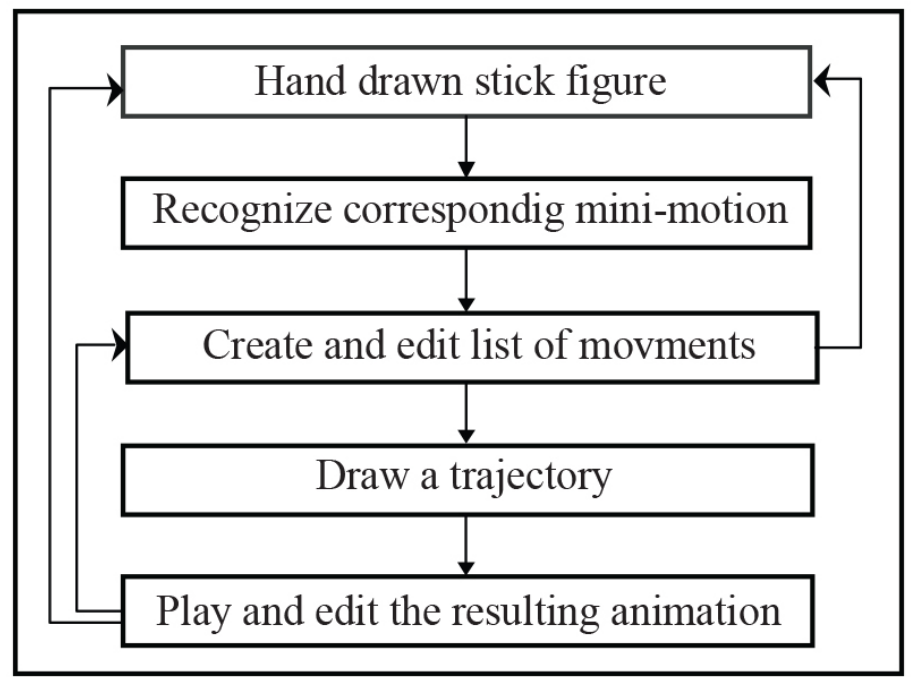

Figure 4.7: The pipeline of sketch-based dance choreography.

a mini-motion. Therefore, the proposed annotations are simpler and closer to the common shape of the stick figures.

Figures 4.8(a), 4.9(a), and 4.10(a) show Zorn's annotations for the Pirouette, Jeté and Rond de jambe movements respectively. To create one of these ballet motions, choreographer must draw every single pose, such as bending the left leg, stretching it, transferring to the other leg etc. For instance, in Figure 4.9(a) a combination of five different annotations (plus a repeat sign) are used to describe poses and movements. While Zorn's system is more understandable than the other notations available, it is

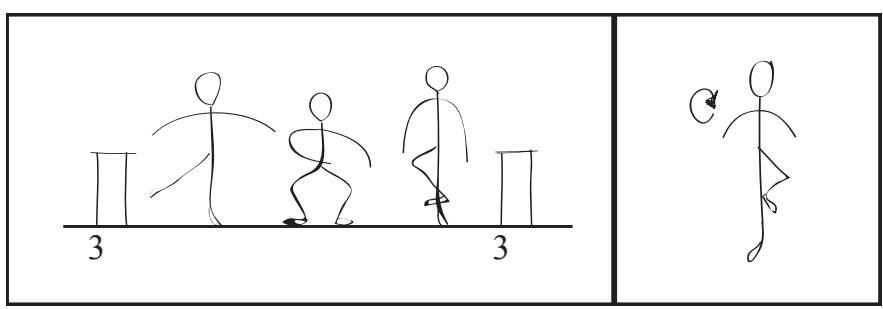

(a)

(b)

Figure 4.8: Pirouette (a) in Zorn Annotation and (b) in new annotation. 


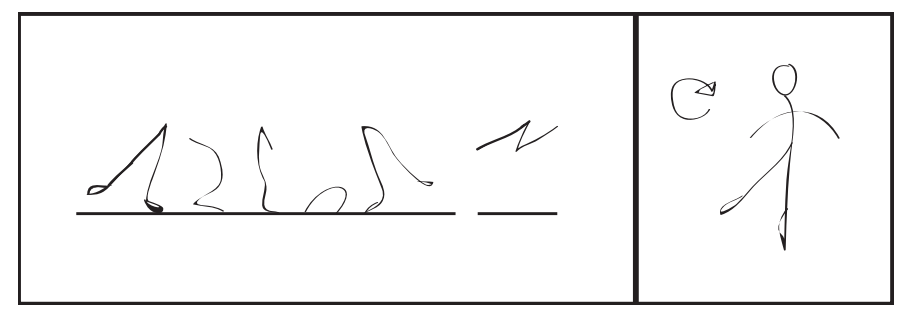

(a)

(b)

Figure 4.9: Jeté (a) in Zorn Annotation and (b) in new annotation.

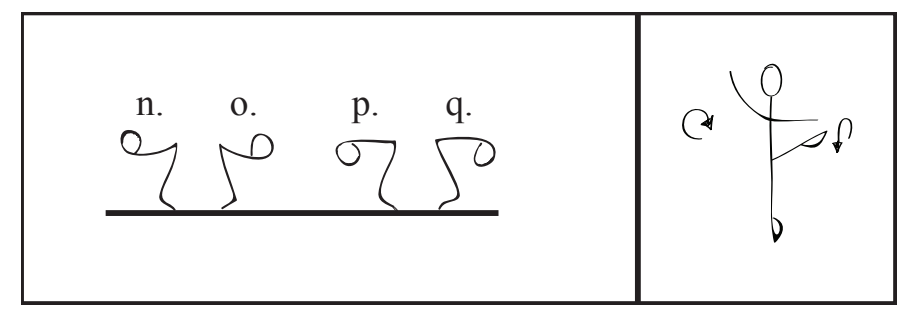

(a)

(b)

Figure 4.10: Rond de jambe (a) in Zorn Annotation and (b) in new annotation.

not efficient to associate all of these annotations to the corresponding mini-motion.

Figures 4.8(b), 4.9(b), and 4.10(b) illustrate the new stick figure annotations that describe the entire sequence of comprising keyframes using only a single representative pose. Instead of showing the details of every movement, this pose captures the main characteristics of the move (according to the vocabulary of ballet). This pose may be accompanied by one or two movement arrows. The curved arrow shows which way the character turns while the straight arrow is used to indicate the direction of movement for the corresponding part of the body. For example, Figure 4.9(b) shows the proposed alternative to Figure 4.9(a) using one representative pose and one movement arrow. These new annotations help choreographers focus on the main features of the motions rather than annotating every detail of the performance. Figure 4.11 shows the list of the main stick figure annotations proposed for this work. 


Cou-de-pied

Figure 4.11: List of the main stick figure annotations proposed for this work.

\subsection{Using Captured Motions}

To create a 3D dance with all the details of a real performance, a set of MoCap data for ballet is required. Connecting these motions together provides sufficient flexibility to create new dance sequences. Capturing classical ballet motions is not easy for MoCap studios because ballet shoes may not work on their floors. This is not an issue for contemporary ballet because it is performed barefoot. The best way 


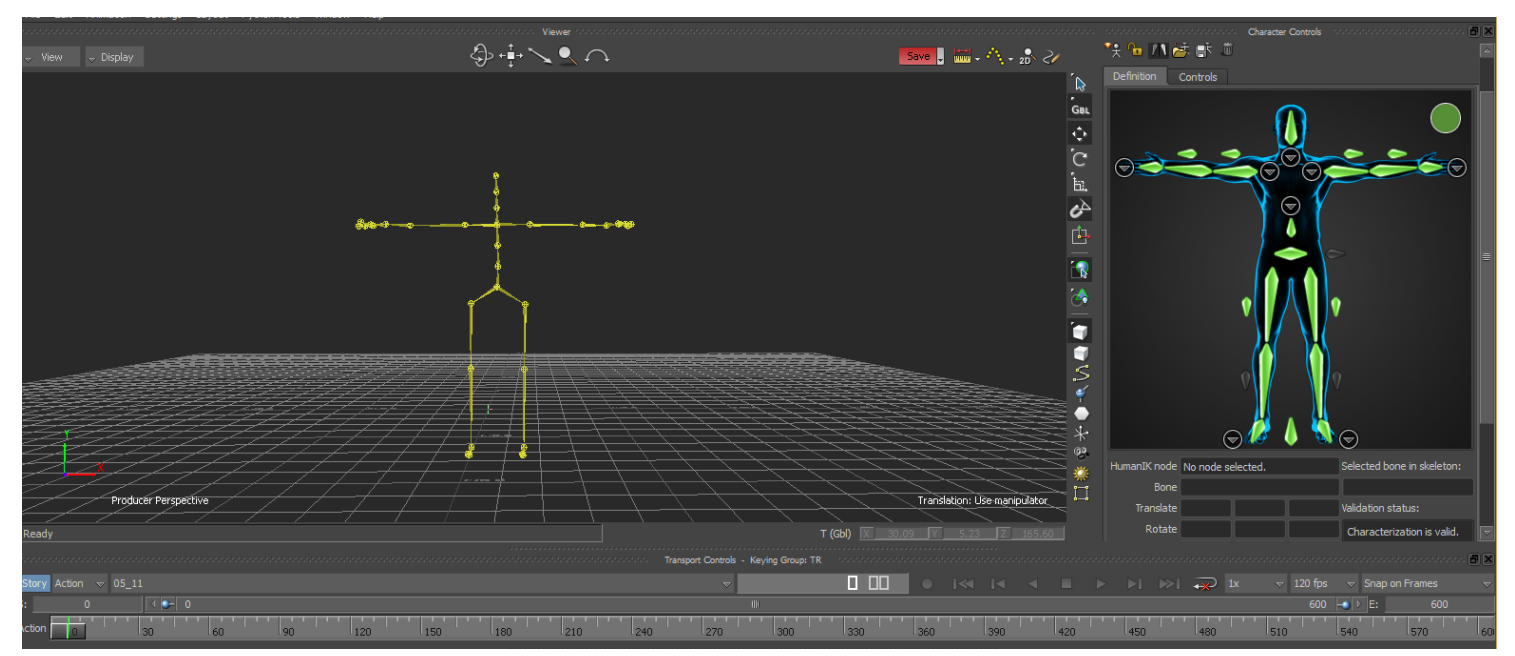

Figure 4.12: Characterization of the raw MoCap data in Motion Builder.

to gather the mini-motions necessary for this approach is to rent a MoCap studio and capture the movements there. For this work, the ballet motions in the Motion Capture Database of Carnegie Mellon University [2] are used. These motions are recorded using 12 Vicon infrared MX-40 cameras that track the movements of 41 markers attached to the body of the subjects. CMU MoCap database contains 2,605 motions in total but only 20 of them are ballet motions.

The gathered motions are first adjusted, cleaned and skinned using Motion Builder [1]. As shown in Figure 4.12, first, the imported MoCap files are characterized to a standard humanoid avatar by manually aligning their joints into a T-Pose. Then, the raw motions are cleaned to avoid footskate and other artifacts. Next, the cleaned motions are re-targeted to a rigged character to represent a skinned dancer in the scene (see Figure 4.13). Finally, the resulting animation is exported to the FBX format which is a well known file format between different animation editing software. Figure 4.14 summarizes this pre-processing pipeline. 


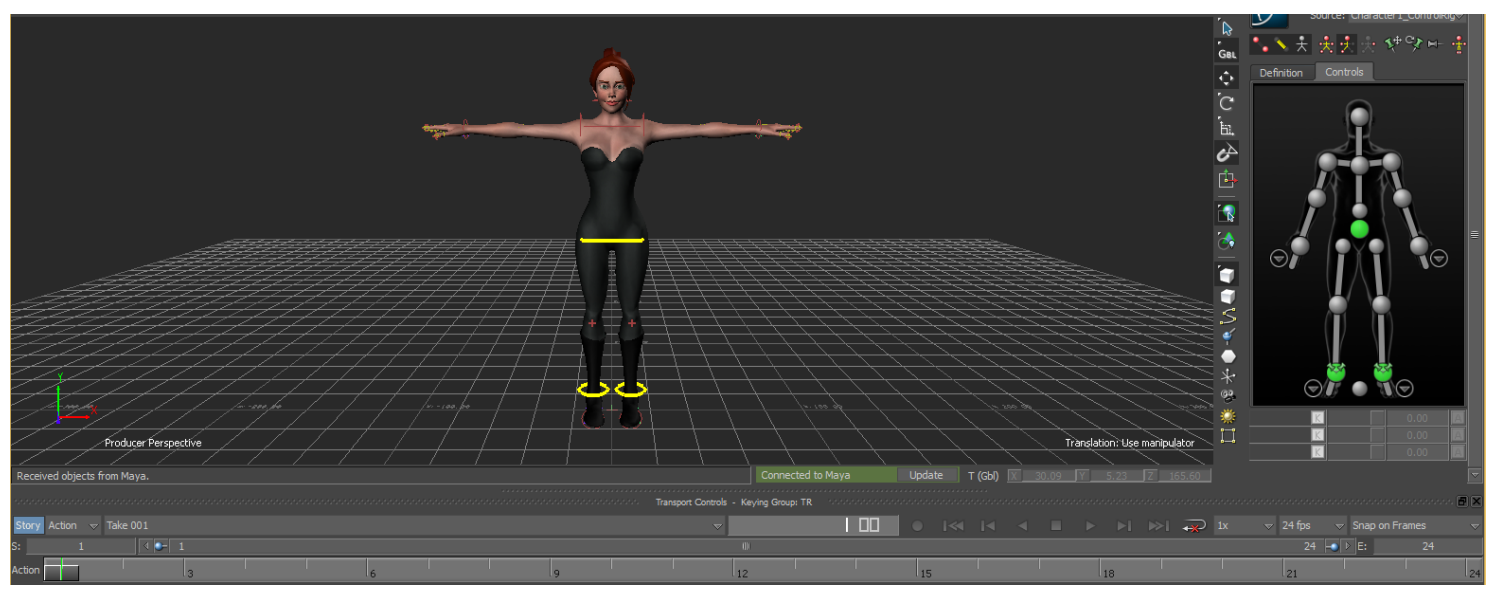

Figure 4.13: Re-targetng the motions to a rigged model.

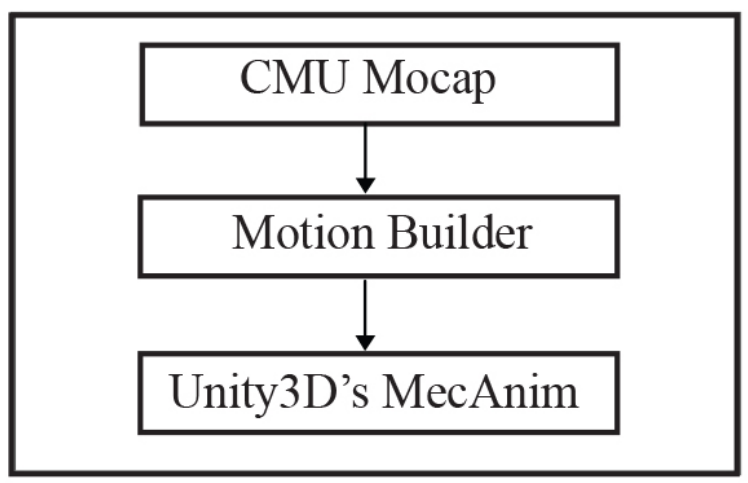

Figure 4.14: The pre-processing pipeline.

\subsubsection{Creating Mini-motions}

Although the quality of the resulting motions are acceptable, these motions contain full ballet dance sequences and need to be segmented into mini-motions. As mentioned in section 2.4, in this thesis, a ballet motion is called a mini-motion (to differentiate it from a ballet sequence). It is important that the start and end frames of a mini-motion $m_{i}$ be matched carefully with the first and last corresponding Zorn annotation. Animation editing tools such as Maya can be used for this purpose, but dynamic animation generation mechanism of Unity3D [3] provides an easy to use ap- 


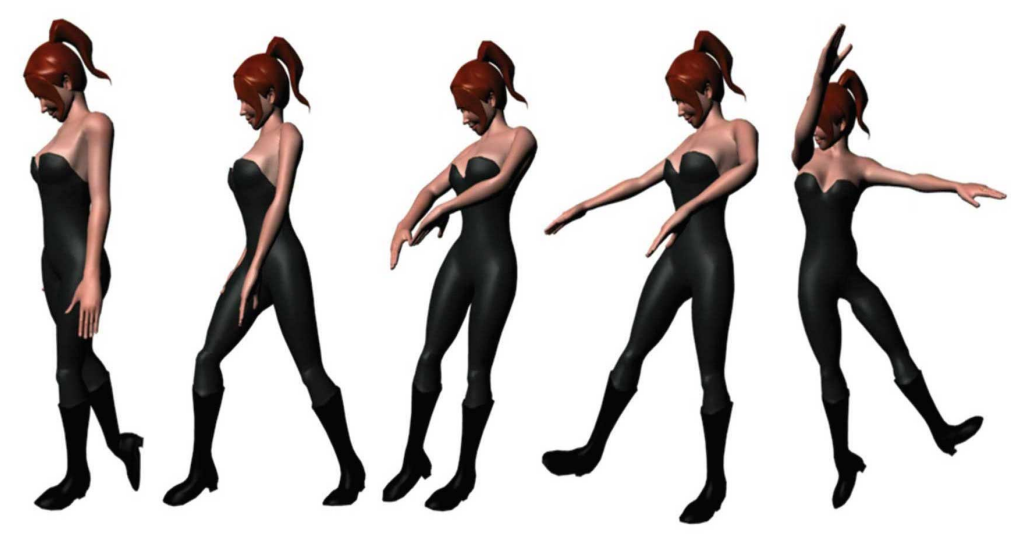

Figure 4.15: The Jeté mini-motion prepared according to the annotations provided in Figure 4.9(a).

proach for motion editing. Therefore, Unity3D's MecAnim is used for segmentation of motion clips into smaller components. Figure 4.15 shows five frames of the Jeté mini-motion carefully extracted from a larger clip. The start and end frames of this mini-motion are visually matched with the corresponding Zorn annotation shown in Figure 4.9(a). These extractions help to provide a basic set of ballet mini-motions $m_{1}, m_{2}, \ldots, m_{k}$ which are stored in a mini-motion repository.

Currently, the implemented prototype has 20 mini-motions that cover only a fraction of the vocabulary of ballet. These mini-motions can be visually inspected and selected from a list. Also, a text-based search is provided to find these mini-motion by entering their names. However, in practical scenarios hundreds of mini-motions are needed that make the visual or text-based selection difficult. This difficulty can be addressed using a stick figure sketch recognition approach. 


\subsection{Sketch Recognition}

The next step, after preparing a set of mini-motions, is assigning their corresponding ballet annotations. To use an sketch-based input, one must be able to retrieve minimotions by sketching their corresponding annotations. This work aims to support freeform strokes, without constraining the input sketch to follow a specific order. Also, to complete the annotations, multiple strokes are usually needed. Finally, because of the branching structure of the stick figures, they do not fall into the category of simply recognizable shapes and need special treatment.

In order to find out which algorithm better detects the drawn annotations with the discussed characteristics, a few gesture recognition algorithms were experimented. \$1 Unistroke Recognizer [61] provides a good algorithm for detecting unistrokes, but the proposed annotations usually require drawing multiple strokes. $\$ \mathrm{~N}$ Multistroke Recognizer [6] addresses this limitation and provides an algorithm that considers all possible stroke orders and directions. This will add more combinatoric overheads that reduces the recognition speed. A version of $\$ N$ utilizing Protractor matching

method [42] improves $\$ N$ 's speed with cost of accuracy. \$P Point-Cloud Recognizer [57] is the latest in the dollar family, that enables both unistroke and multistroke recognition without the combinatoric overhead of $\$ N$. It recognizes unordered point-clouds in a low-cost, accurate and fast way.

As shown in Figure 4.16, the number, order and direction of the strokes used fro drawing a simple square are ignored when looking at the square as a cloud of points. This point cloud representation enables $\$ P$ to recognize both unistrokes and multistrokes equivalently. Using an approximation of the Hungarian algorithm [38], \$P 

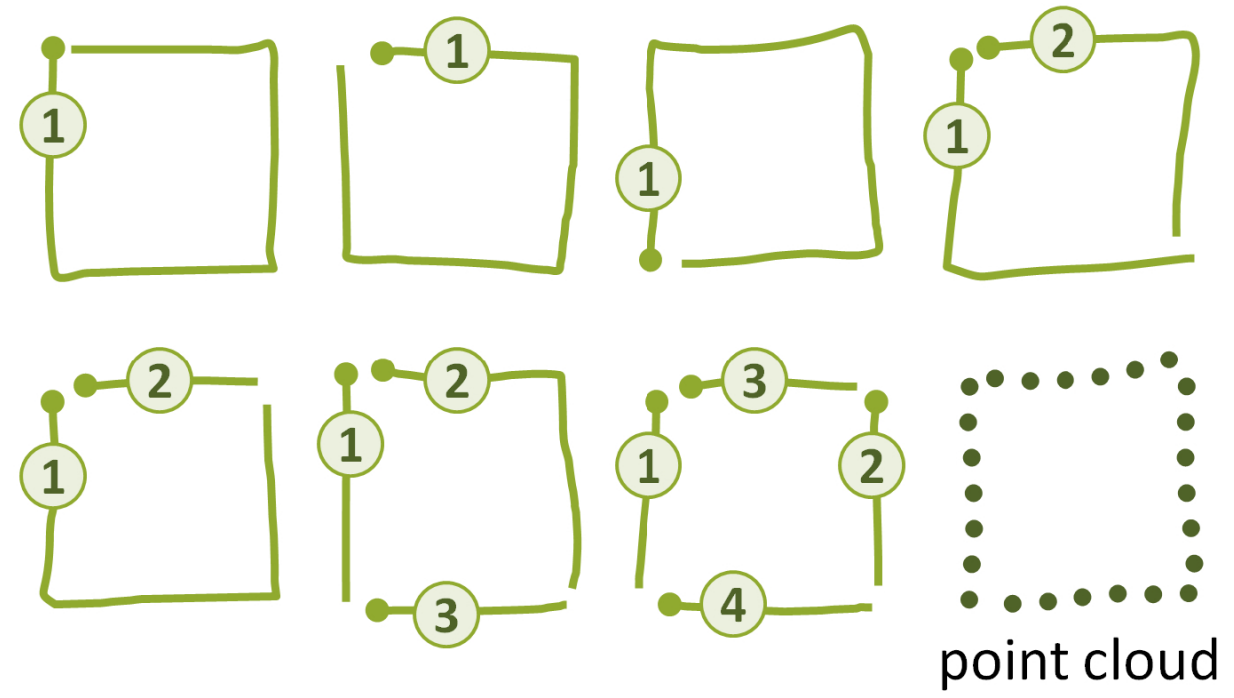

Figure 4.16: The various ways of drawing a square do not matter in the resulting point cloud. Image taken from [57].

solves the classic assignment problem between two bipartite graphs to compare two point-clouds. With a nearest-neighbor classifier using a Euclidean scoring function, the point cloud of the given candidate gesture $(C)$ is matched to the point cloud of a template $(T)$ stored in the training set. To compute the matching distances, both $C$ and $T$ should be normalized to the same number of the points. Because of the acceptable results of the $\$ \mathrm{P}$ Point-Cloud Recognizer it is chosen for the proposed approach. To reduce the error rate, 10 different templates for each annotation are stored in the training set. These templates follow no constraints on the number of the strokes, stroke ordering and stroke direction. Based on the recognized annotation, one of the mini-motions $m_{1}, m_{2}, \ldots, m_{k}$ is retrieved. 


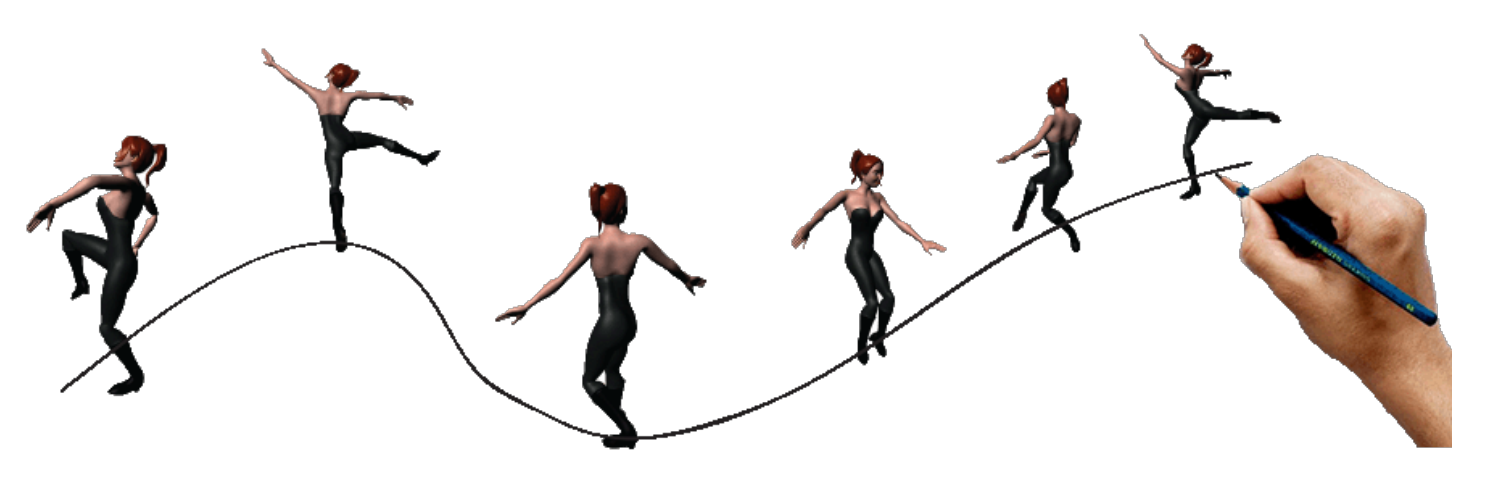

Figure 4.17: A sequence of dance following the sketched trajectory.

\subsection{Sketch-Based Dance Choreography}

In the real world, dancers change their motions dynamically. They combine a few mini-motions together to create a dance pattern. The proposed approach tries to simulate the same process using animation blending. It starts by drawing a series of sketched annotations which retrieve a sequence of mini-motions $m_{i_{1}}, \ldots, m_{i_{l}}$ where $i_{1}, \ldots, i_{l} \in\{1, \ldots, k\}$. After the mini-motions are successfully retrieved, they can be re-ordered or re-arranged if necessary. This can be done by adding retrieved minimotions to a list of movements. This list can then be re-arranged to achieve the best order of dances in the scene. To play the dance in the scene, the choreographer needs to specify the main trajectory of the dancer by drawing a path $p(u)$ on the floor of the 3D space, similar to the Feuillet system [23]. Mini-motions are then blended together and played along this path in the same order of their appearance as in the list of movements. This allows one to preview and evaluate the synthesized dance from different angles. Figure 4.17 shows a dance sequence created using this approach with 4 mini-motions, which follows the given path. 


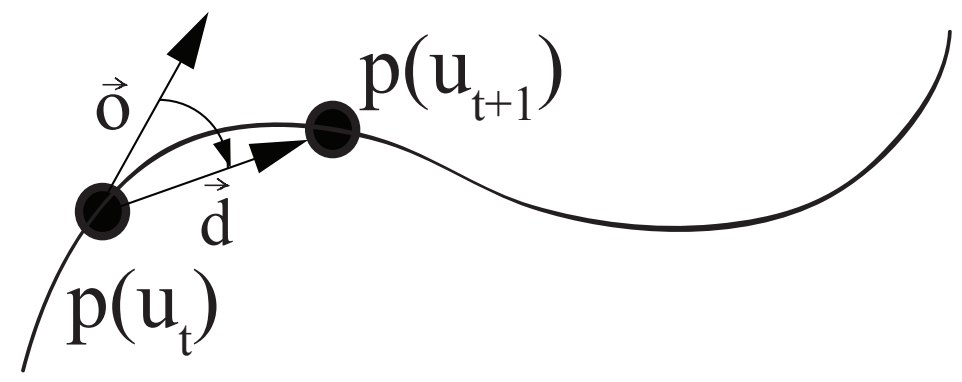

Figure 4.18: To align the direction of the dancer on the path's current direction, the dancer is tilted towards the approximate tangent.

To create the trajectory $p(u)$, the intersection points between the floor plane and the rays casted along the view direction from the current mouse position are found. This path is then denoised and parameterized. As indicated in Figure 4.18, to align the motion along the sketched path, first the current direction $\vec{O}$ of the avatar is found, using the quarternion rotation of its root joint at $p\left(u_{t}\right)$. Then, the avatar's direction is tilted toward the tangent direction $\vec{d}$ approximated by $p\left(u_{t+1}\right)-p\left(u_{t}\right)$. The cross product $\vec{o} \times \vec{d}$ is used at each frame to calculate the dancer's tilt angle. By aligning the direction of the root joint at $p\left(u_{t}\right)$ with the given path, the footsteps will follow the path automatically. This direction control mechanism works best when mini-motions are not lengthy. Otherwise, the character may go slightly off the track because it will be tilted when a clip is finished playing.

To animate a smooth dance, the mini-motions need to be blended smoothly. For this purpose, the state machine mechanism of the Motion Graphs [37] is used. As discussed in chapter 2, Motion Graphs transform the motion synthesis problem into a graph walk. The MecAnim Animation System of Unity3D [3] is based on similar technique. Figure 4.19 shows a portion of the states and transitions that were created for this project using MecAnim. A sample transition is shown in Figure 4.20 that 


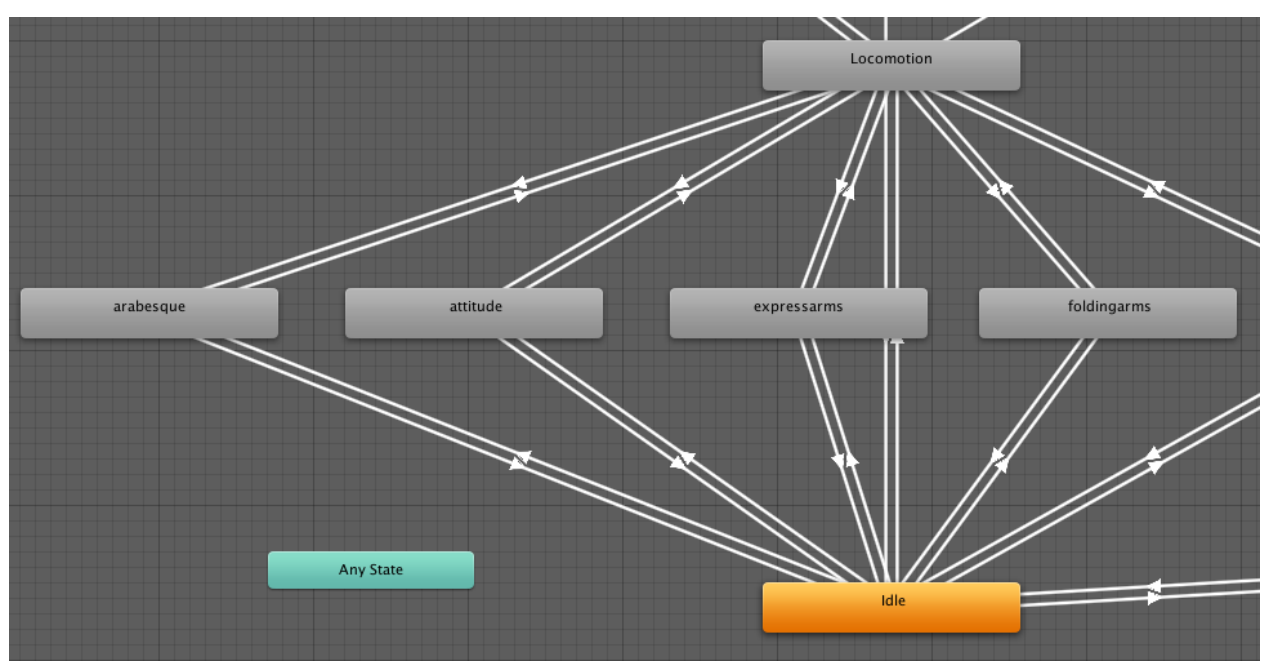

Figure 4.19: Unity3D's state machine is used to blend different mini-motions.

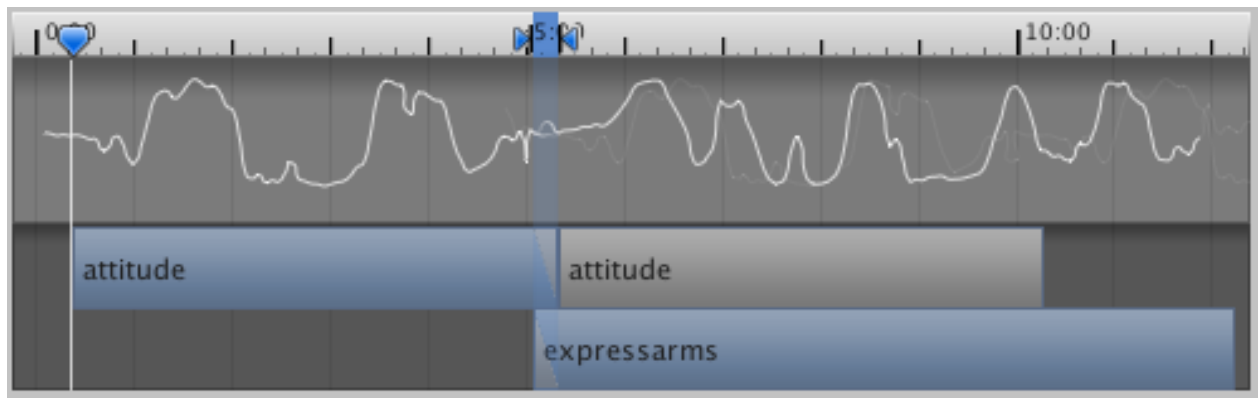

Figure 4.20: Blending two mini-motions using linear interpolation.

linearly blends the end frames of mini-motion 'attitude' to the starting frames of the succeeding mini-motion 'expressive arms'. To avoid visual discontinuity between footsteps of different mini-motions, they are adjusted to playback smoothly for each mini-motion. Finally, the path and sequence of motions can be saved for later use.

\subsubsection{Limitations}

As mentioned in section 4.4.1, one of the limitations of the current approach is the small number of mini-motions in the database. The current mini-motions cover only 
a fraction of the ballet vocabulary. This prevents supporting all dance sequences performed in real ballet dance works. Also, regardless of all the efforts spent on cleaning and skinning the motions in the database, they sometimes show unwanted artifacts. Finally, if the sketched trajectory contains sharp corners, it takes some time for the 3D avatar to adjust its direction and come back to the main trajectory.

\subsection{Conclusion}

To conclude the proposed approach for dance choreography, the steps of the methodology are summarized. First, an initial mini-motion is assigned to the character by sketching a stick figure annotation that is inspired from Zorn annotation system. This new annotation system simplifies different steps in just one single stick figure. To assign a mini-motion to each annotation, a database of ballet movements is created using Carnegie Mellon University's MoCap database. Also, \$P Point Cloud Recognizer is used to match the drawn annotation to the corresponding mini-motion

in this database. After retrieving the initial mini-motion from the motion database, choreographer can preview and evaluate it from different angles by placing it on the dance floor. Choreographer can sketch the main trajectory of the dancer in the scene. This path will be used to place mini-motions that choreographer will choose repeatedly. This proposed method of dance creation greatly helps choreographers to compose innovative and believable dance patterns. 


\section{Chapter 5}

\section{Results}

As discussed in chapter 4 , having a 3D sketch-based environment for choreography helps to compose and edit dance steps in the same way done by choreographers. Drawing a simple stick figure annotation, is an intuitive way to retrieve desired mini-motions from the database. Also, blending a set of selected mini-motions along a drawn path is an interactive way to represent dance motions with all of the details of a real performance. This new approach for dance creation can help choreographers to compose innovative and believable dance patterns. To support the effectiveness of this approach, a sample prototype was designed for this project. Then the design materials were given to a computer scientist to build the prototype using Unity3D game engine and C\# scripting language. This chapter provides more details about the user experience with this prototype and presents its outcomes. It also evaluates the results of the proposed approach with the standard practices.

\subsection{Prototype}

The design of a prototype for the proposed approach was started from the storyboards illustrated in section 4.2. Unity3D game engine was chosen for the development of the prototype because of its powerful animation blending mechanism. However, Unity3D provides limited features for creating user interfaces. Therefore, some of the original concepts were simplified in the final prototype. In designing the 


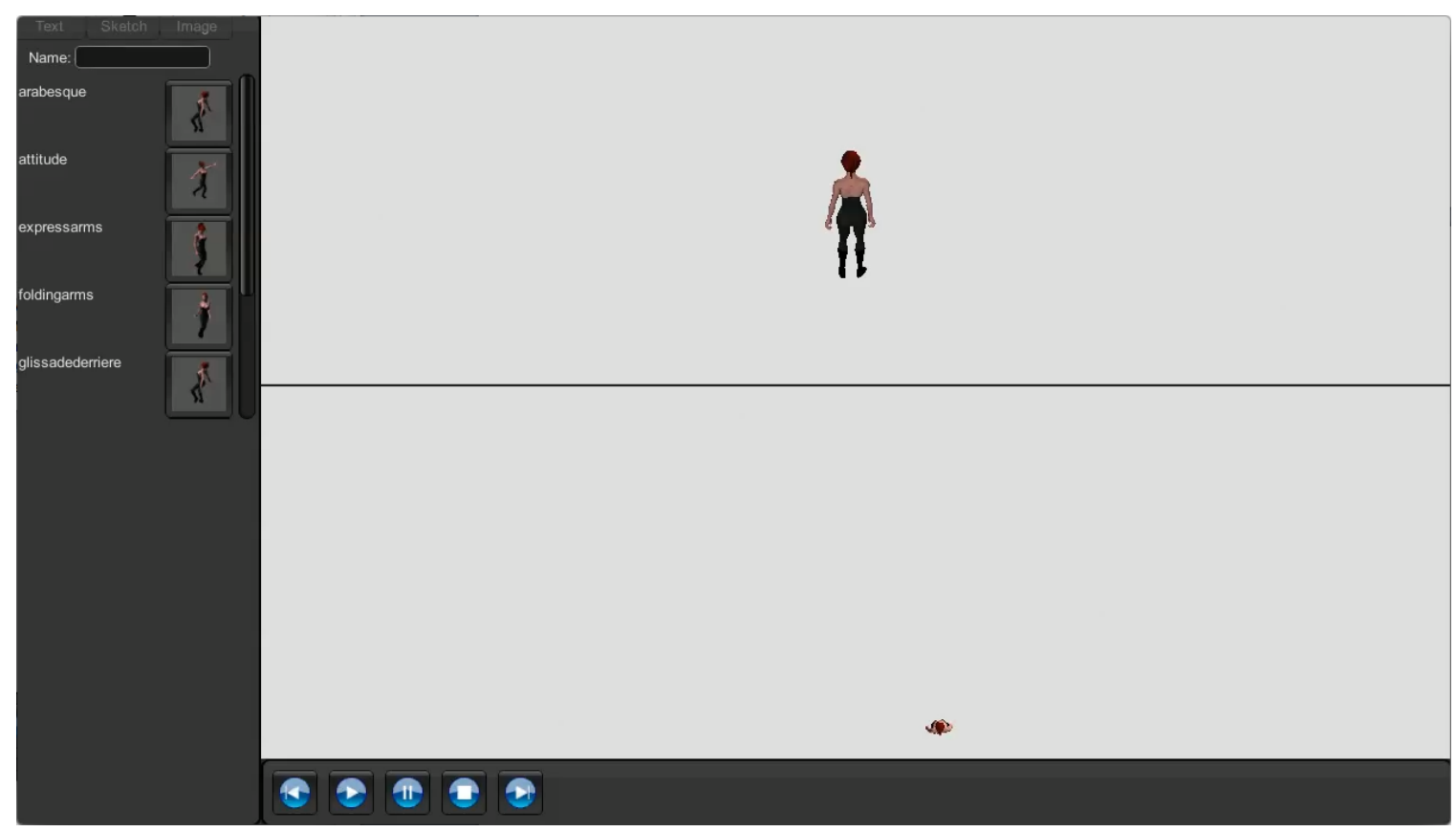

Figure 5.1: The user interface of the first prototype created for this thesis.

workflows for user interaction with the system the core principals of user-centered design and field study discussed in section 4.1 were considered. Also, to meet the best practices of the sketch-based interfaces, no menubar was used and the use of buttons were minimized. Working closely with a computer scientist, these concepts were developed using $\mathrm{C \#}$ scripting language.

Figure 5.1 shows a screenshot of the first prototype created to support the approach discussed in this thesis. Mini-motions are represented by their names and icons in the left panel. Also, this panel provides an input box to search for these mini-motions by entering their names. Each entry in this list represents a FBX file that has been pre-processed as discussed in section 4.4. These files are initially added to a state machine created using Unity3D's MecAnim. Selecting each icon will add the corresponding FBX file into the list of movements. 

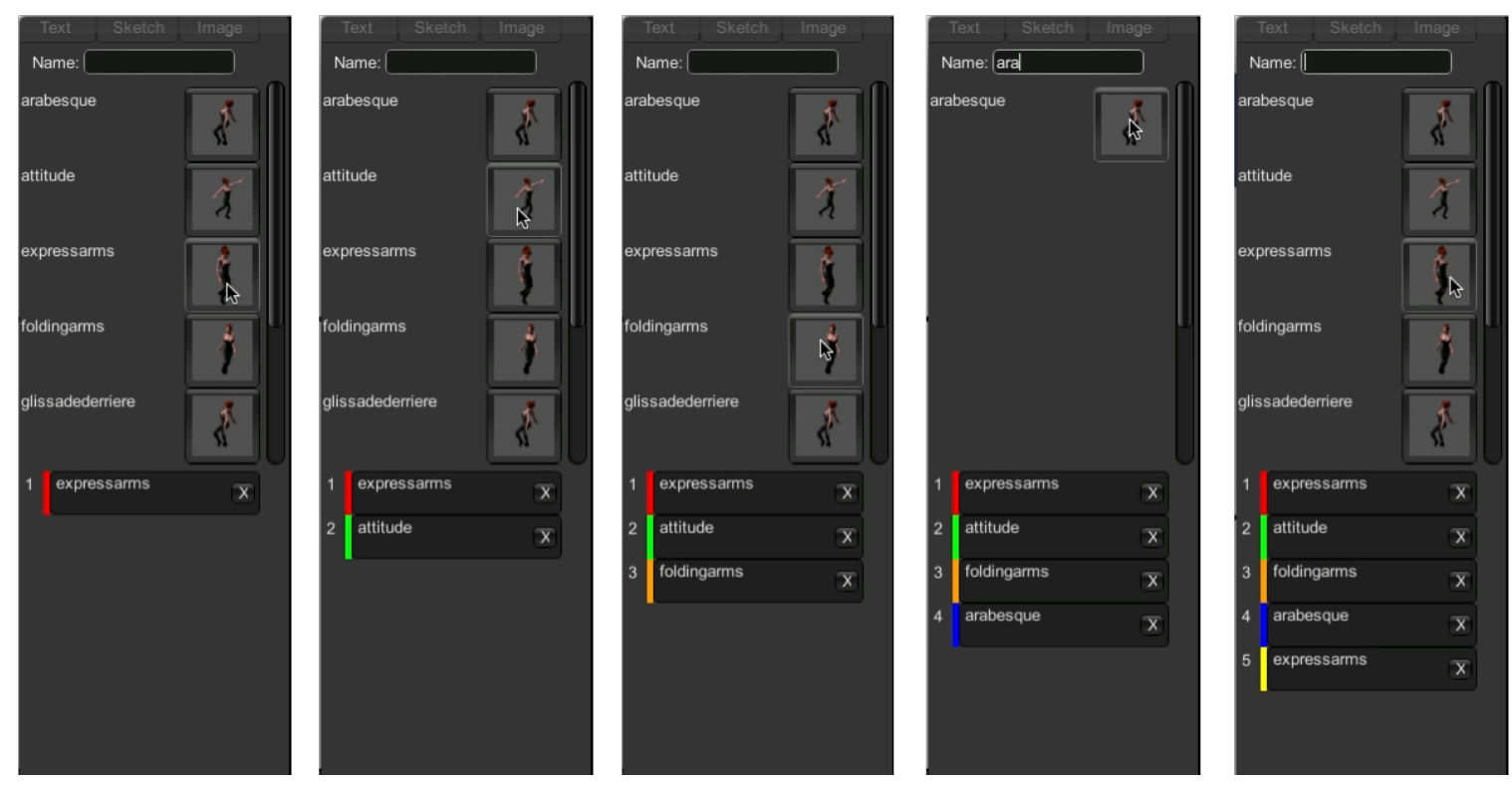

Figure 5.2: The process of adding mini-motions to the movement list.

Figure 5.2 shows the process of selecting, searching and adding mini-motions to the list of movements. Mini-motions in this list are represented with different color codes. This list will determine the sequence of mini-motions that should be blended together. As shown in Figure 5.3, it is possible to change the order of the minimotions in this list by dragging and dropping them. Also, by clicking on the cross mark associated with each entry, it can be removed from this list.
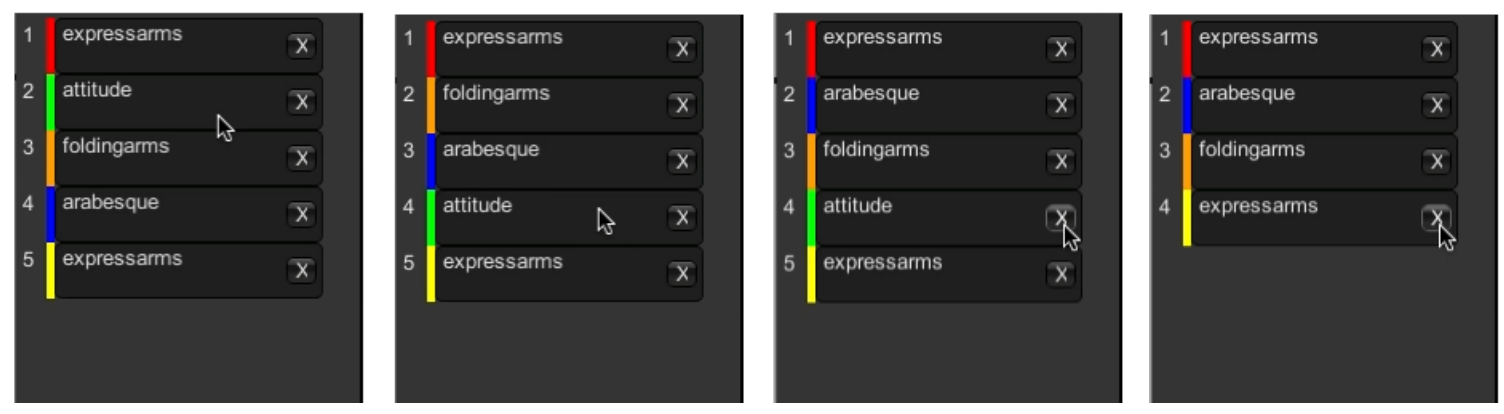

Figure 5.3: The process of changing the order of attitude mini-motion in the movement list and removing it from the list. 


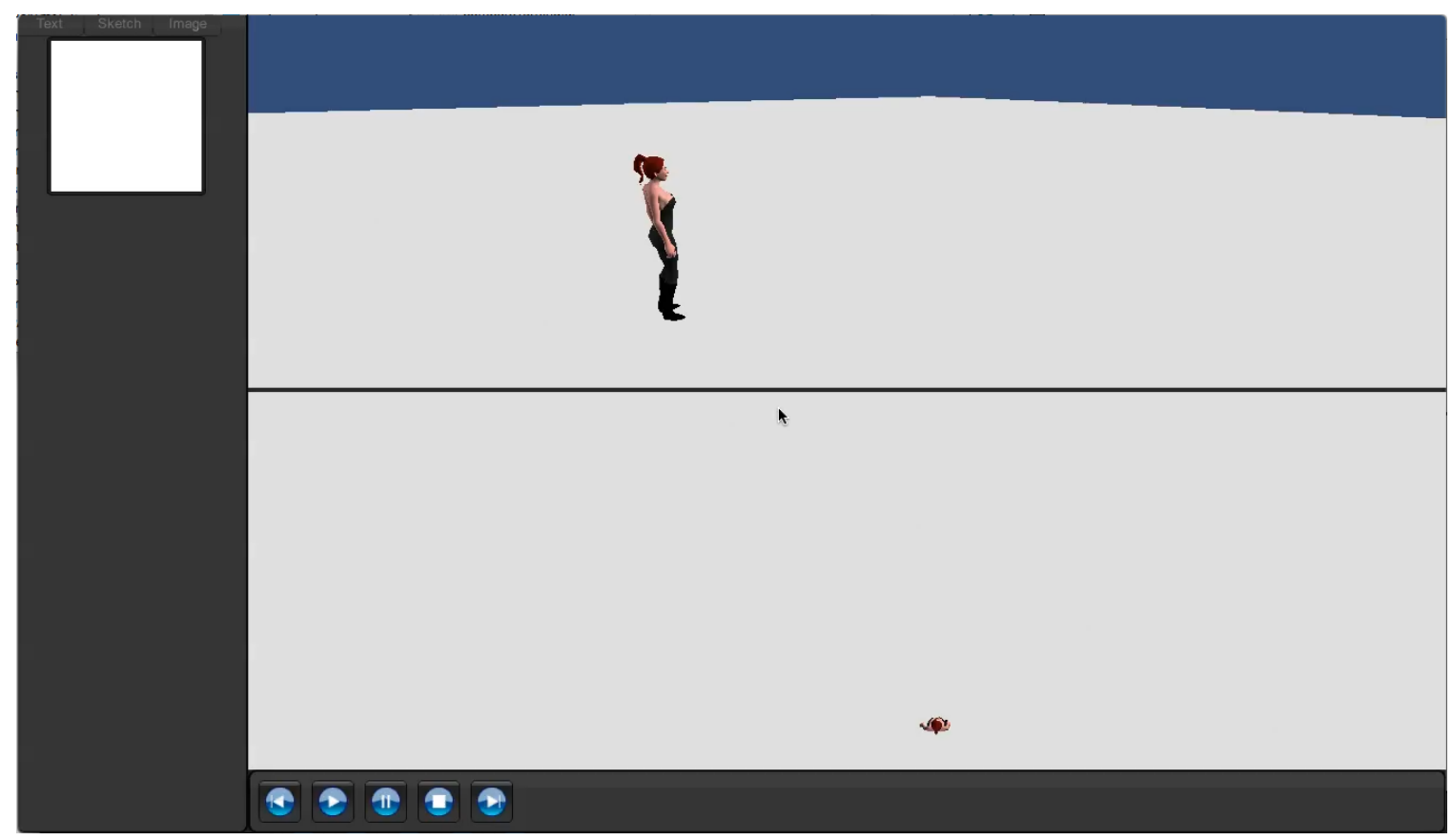

Figure 5.4: The user interface of the final prototype created for this thesis.

The first prototype is useful when the number of mini-motions is limited and there is a good knowledge about them. However, this is not usually applicable to real case scenarios with hundreds of mini-motions. To address this limitation, the prototype was enhanced to support sketching the annotations described in section 4.3. As shown in Figure 5.4, the sketch panel allows choreographer to focus on composing dance steps by drawing their annotations. Using the sketch recognition technique discussed in section 4.5 , the mini-motion that has the closest annotation to the given sketch can be found. If the mini-motion is successfully retrieved for the sketch, the corresponding icon can be added to the list of movements. Figure 5.5 illustrates this process of sketching representative poses and adding the retrieved mini-motions to the list of movements. It represents adding two instances of the attitude mini-motion and one instance of the folding arms mini-motion to the movement list. 

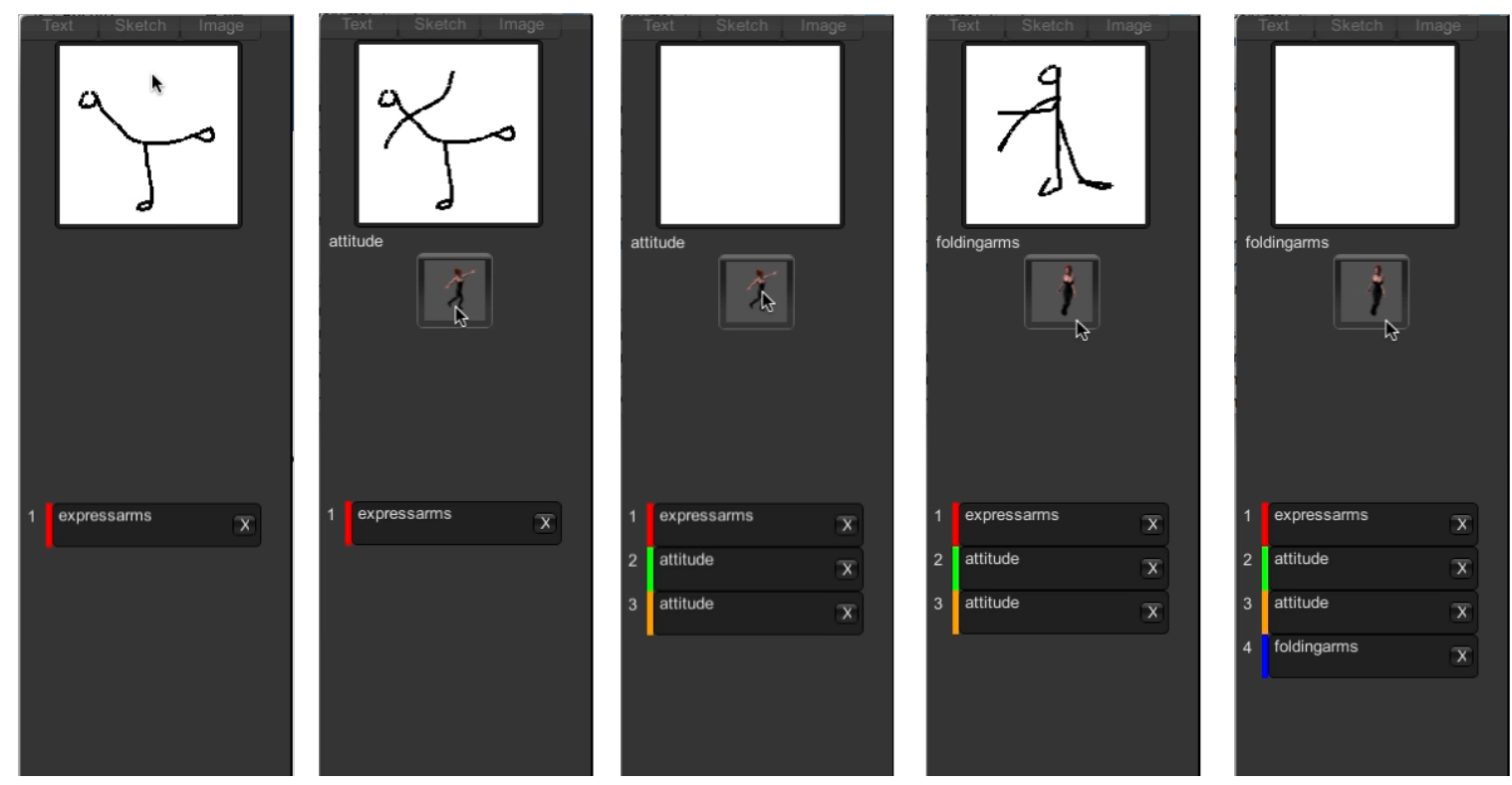

Figure 5.5: The sketch-based process for adding mini-motions to the movement list.

As shown in Figure 5.6, when choreographer finished editing mini-motions in the list of movements, the trajectory of the dancer can be drawn interactively in the top view window. This raw trajectory is denoised and parameterized and will be shown in both perspective view and top view. Then, by hitting the play button in the playback bar, the dynamic animation mechanism of Unity3D starts blending minimotions as well as playing the music score. In this prototype, the music score only plays as a background sound and does not control the timing of the mini-motions.

In order to blend mini-motions along the given trajectory, the direction of the avatar will be tilted at each frame toward the path using the technique discussed in section 4.6. At each frame, the dancer is represented both in the perspective view and the orthogonal view. It allows choreographer to rotate the camera, zoom it or pan it in each view to see the dance from arbitrary angles. Also, it is possible to save the complete choreography as an animation clip for future use. A YouTube video 


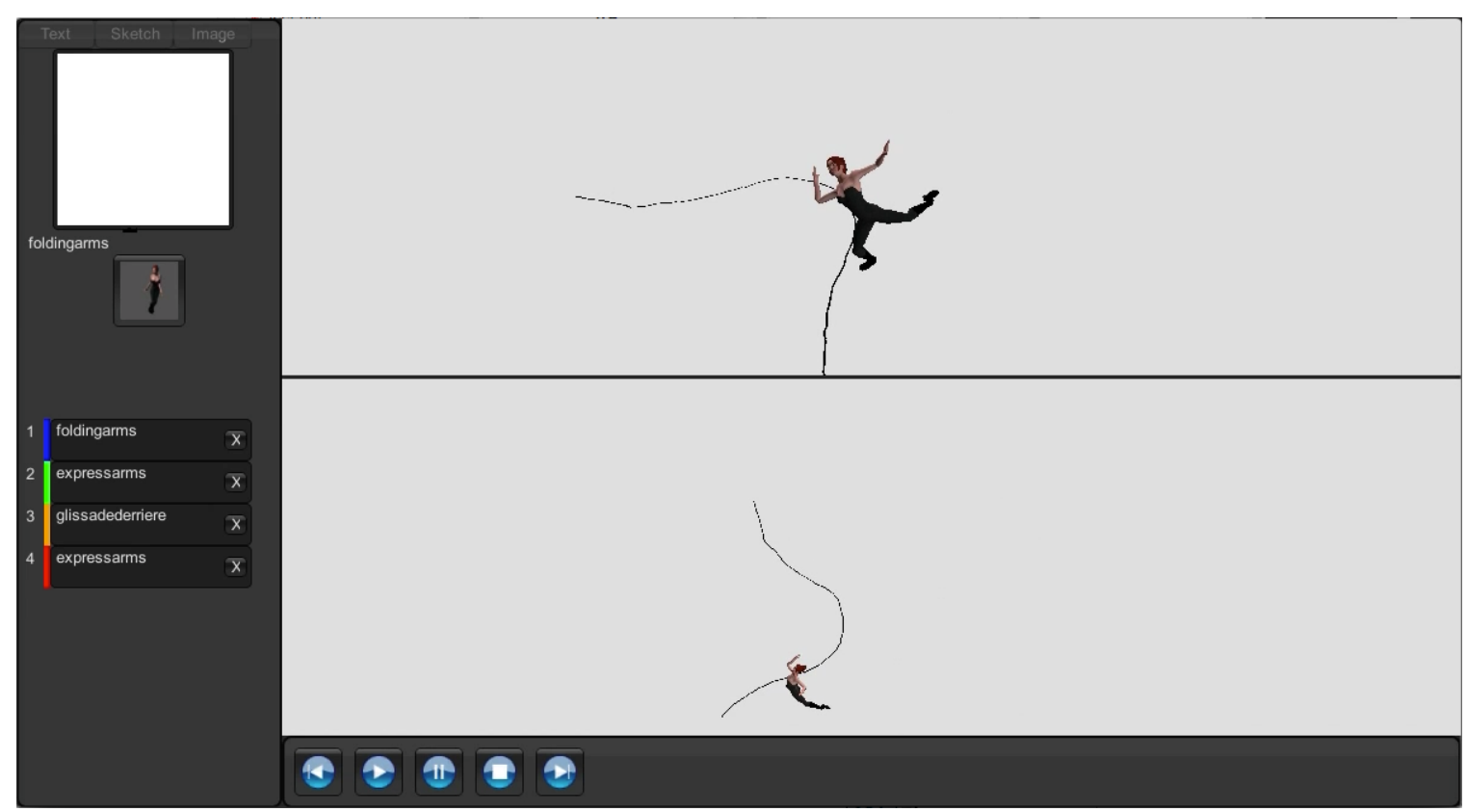

Figure 5.6: The process of directing the dancer by drawing a trajectory.

is prepared to demonstrate this process which can be watched from this address: http://youtu.be/JKDd2bg08-k.

\subsection{Applications}

The simple and natural user interface introduced in section 5.1 converts the timely process of dance annotation into a fast and interactive experience. Animated avatar in a 3D environment provides more information than other medias. It allows people to observe and learn the dance steps from different angles as visualized in Figure 5.7 . Also, Figures 5.8 to 5.10 represent blended mini-motions based on different annotations listed in Figure 4.11. A sequence of screenshots taken from a good angle can be used for traditional media such as books. This can save a lot of time in drawing and publishing the training materials. Additionally, this approach may provide a bet- 


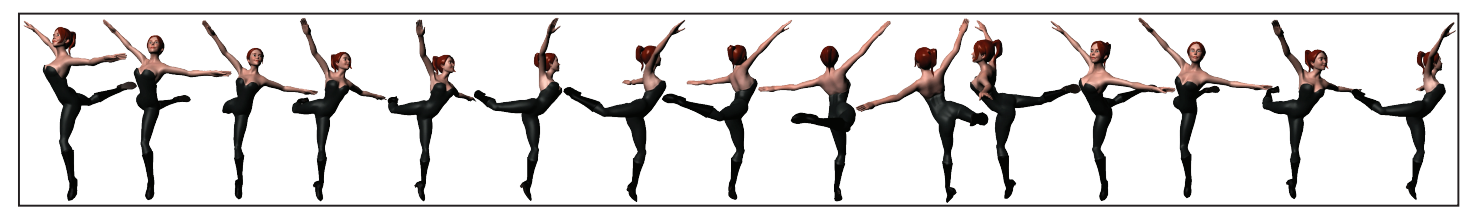

Figure 5.7: The Attitude pose shown from different angles.

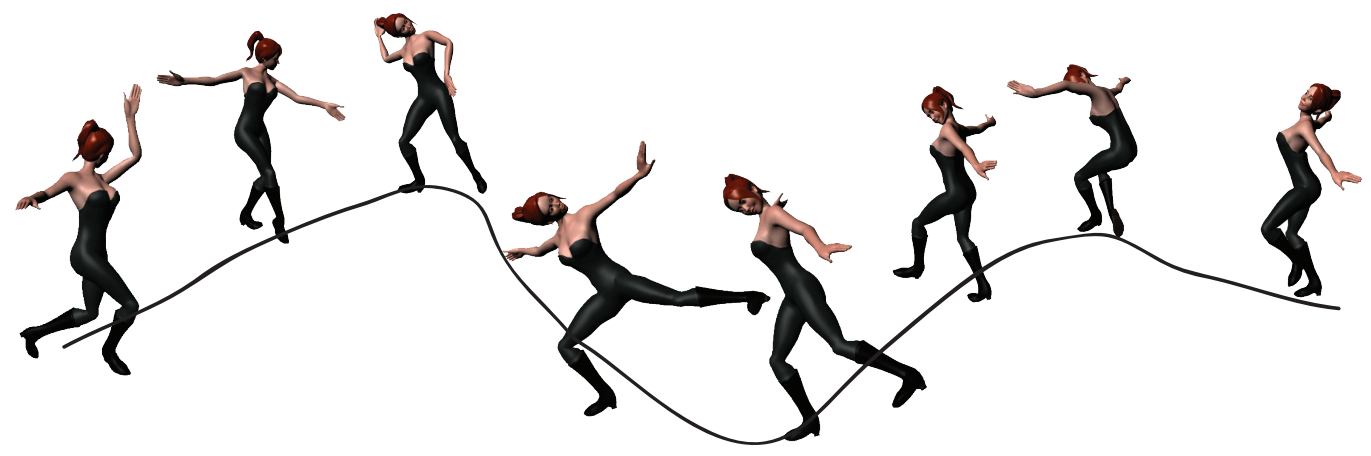

Figure 5.8: Combination of Arabesque, Turn step and Folding arms mini-motions.

ter learning mechanism specifically for children since they love to watch animated characters.

\subsection{Evaluation}

In order to evaluate the proposed approach, sample representations of the new annotation system is compared against other annotations that are commonly used for ballet choreography. 


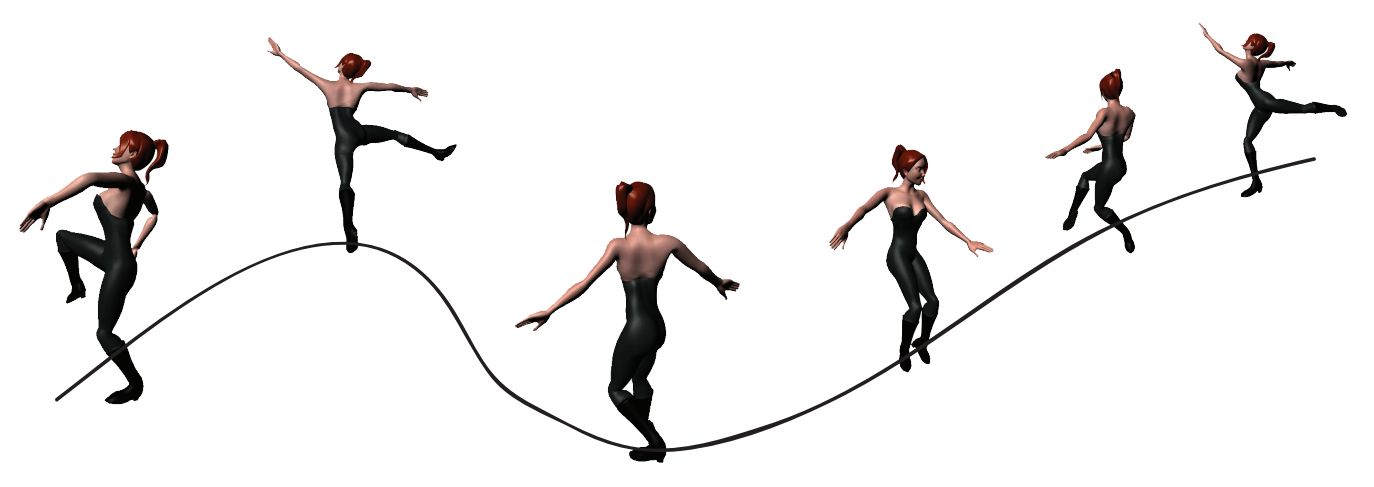

Figure 5.9: Combination of Quasi cou-de-pied, Raised leg above hip height and Jete en touran mini-motions.

\subsubsection{Comparison of Feet Position}

Figure 5.11 compares representation of the 5 major feet positions of ballet in 5 wellknown annotation systems with the proposed representation.

In Feuillet, for representation of the foot position, the foot is indicated by a white pin. The point of this pin represents the toe and the white circle shows the heel. Using this convention, position of the feet are easily and pictorially indicated.

In Zorns annotation, the indication for positions of the feet are more pictorial. They are represented in a frontal view while Feuillet uses an orthogonal view. Also, to avoid ambiguity, a dot is added to show which foot is behind. The second row of Figure 5.11 shows the foot positions in demi-plie and releve poses as well.

In Benesh movement notation, a wide horizontal line below the staff lines indicates that the feet are together, e.g. $1^{\text {st }}$ feet position. In the $2^{\text {nd }}$ position, they are shown to be apart. In the $3^{\text {rd }}$ position, the left foot is shown to be partially behind the right foot, by placement of a black dot for the behind foot. In the $4^{\text {th }}$ position, 


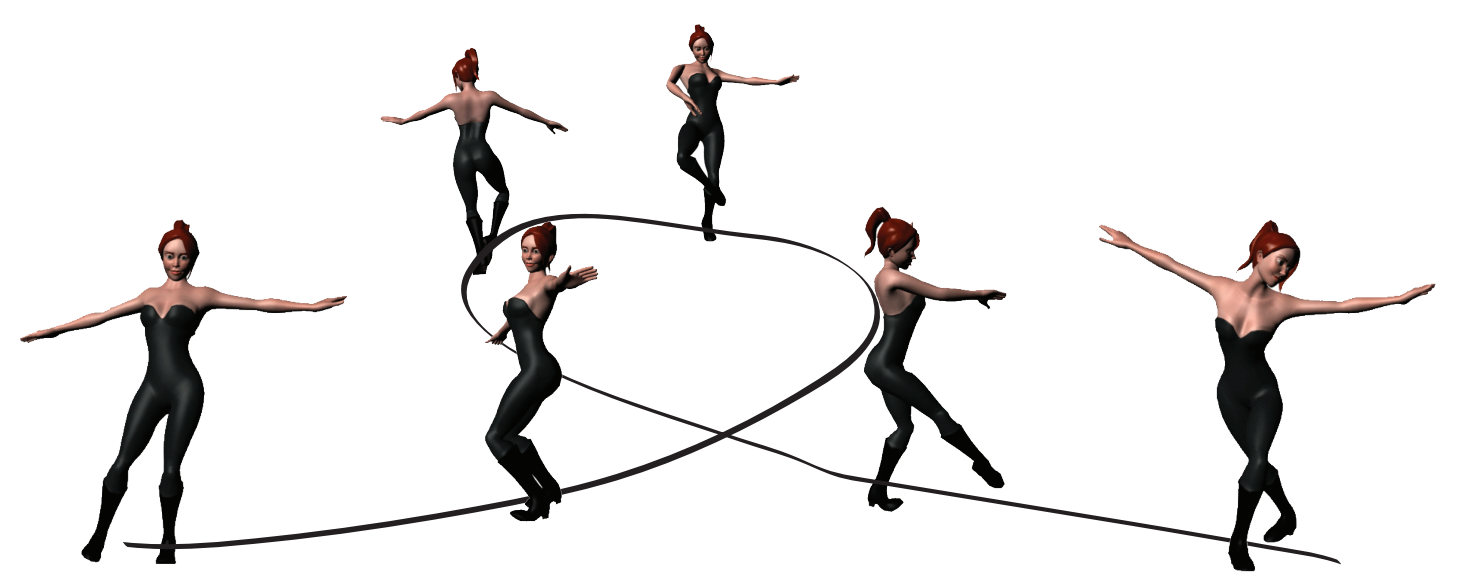

Figure 5.10: Combination of Sideways steps and Pirouette mini-motions.

the right foot is forward and the left foot is backward while in the $5^{\text {th }}$ position the left foot is shown to be completely behind the right. For the demi-plie, the knees are shown to be bent and out to the sides, lower than normal knee height. For releve, the sign is placed on top of the bottom line which means it is supported on the half-toe.

In the Stepanov notation, both feet are in place in the $1^{\text {st }}$ position while the square note indicates contact with the ground. In the $2^{\text {nd }}$ position, each leg is to the side. For the $3^{r d}$ position, the indication is similar to the $5^{\text {th }}$ position but with the diminished signs added. The $4^{\text {th }}$ position shows right foot forward and the left foot backward. Finally, the $5^{\text {th }}$ position shows the feet in place but with the right foot in front. In demi-plie, feet are together with the knees bent which is indicated by adding strokes for bending across the stem of the note. In releve, weight on half-toe is started by two vertical strokes next to the indicator of the $1^{\text {st }}$ position.

In Laban's notation, position of the feet are shown using different symbols while black pins are added to show the relationship of one foot in front of (or behind) the other. Diagonal pins are used for the $3^{\text {rd }}$ position and forward and backward pins 


\begin{tabular}{|c|c|}
\hline Feuillet & 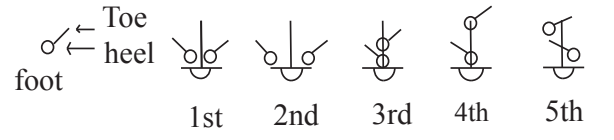 \\
\hline Zorn & 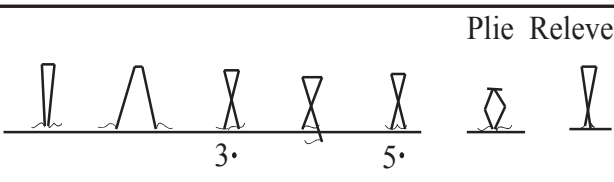 \\
\hline Benesh & 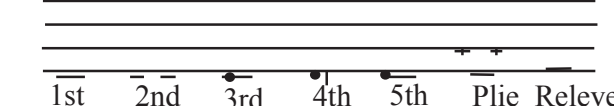 \\
\hline Stepanov & 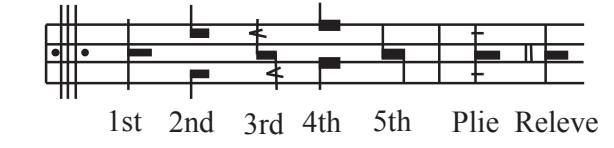 \\
\hline Laban & 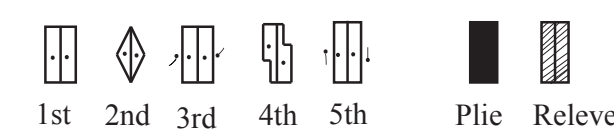 \\
\hline $\begin{array}{l}\text { Proposed } \\
\text { approach }\end{array}$ & 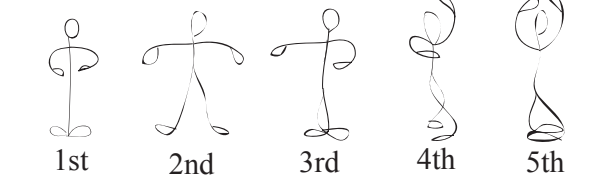 \\
\hline
\end{tabular}

Figure 5.11: Comparing feet position between the common annotation systems.

are used for the $5^{\text {th }}$ position. In the $2^{\text {nd }}$ position, each foot is sideward of the plumb line. In the $4^{\text {th }}$ position, the right foot is sideward and the left foot is backward of the center. The low support in demi-plie compared to the $1^{\text {st }}$ position indicates the knees are bent and the raise of state in releve compared to the $1^{\text {st }}$ means it is on the half-toe.

The notations used for representation of the foot position in the proposed approach are similar to the Zorn's annotation. They provide simple and natural rep- 
resentations that can be understood easily. They also benefit from the upper body positions for disambiguation.

\subsubsection{Comparison of Arm Position}

Figure 5.12 compares movement of the arm in 5 well-known annotation systems with the proposed representation.

In Feuillet, movement of the arms usually follow a circular pattern (semi-circle or full-circle) in an inward or outward direction. First row for Feuillet's notation, shows these circles for the hand which starts from a semi-circle downward. It then shows an upward and an inward full circle. It finally shows a downward and outward full circle. The same patterns are shown for the lower arm in the second row and whole arm in the third row. In Feuillets annotation, the use of upward full arm circles are rare.

In Zorn's annotation, the head, shoulders and central line of the spine are drawn in a frontal view. Thus the arm movements are quite pictorial. In addition, use of dotted lines makes the movement paths easier to see.

In Benesh notation, the arms start down at the sides of the body. The lateral paths of the arms are quite easy to follow in the first measure. In the second measure, the forward path of the right arm is shown by the horizontal tick across the movement line. The right arm lowers to the side of the body where it started before rising sideward to the right. The end line movement lines show the left arm finishing up above the shoulder and the right arm out to the side at shoulder height.

Stepanov's annotation also starts with both arms down. In the first measure the right arm moves to side horizontally, then up to the high place. In the next measure 


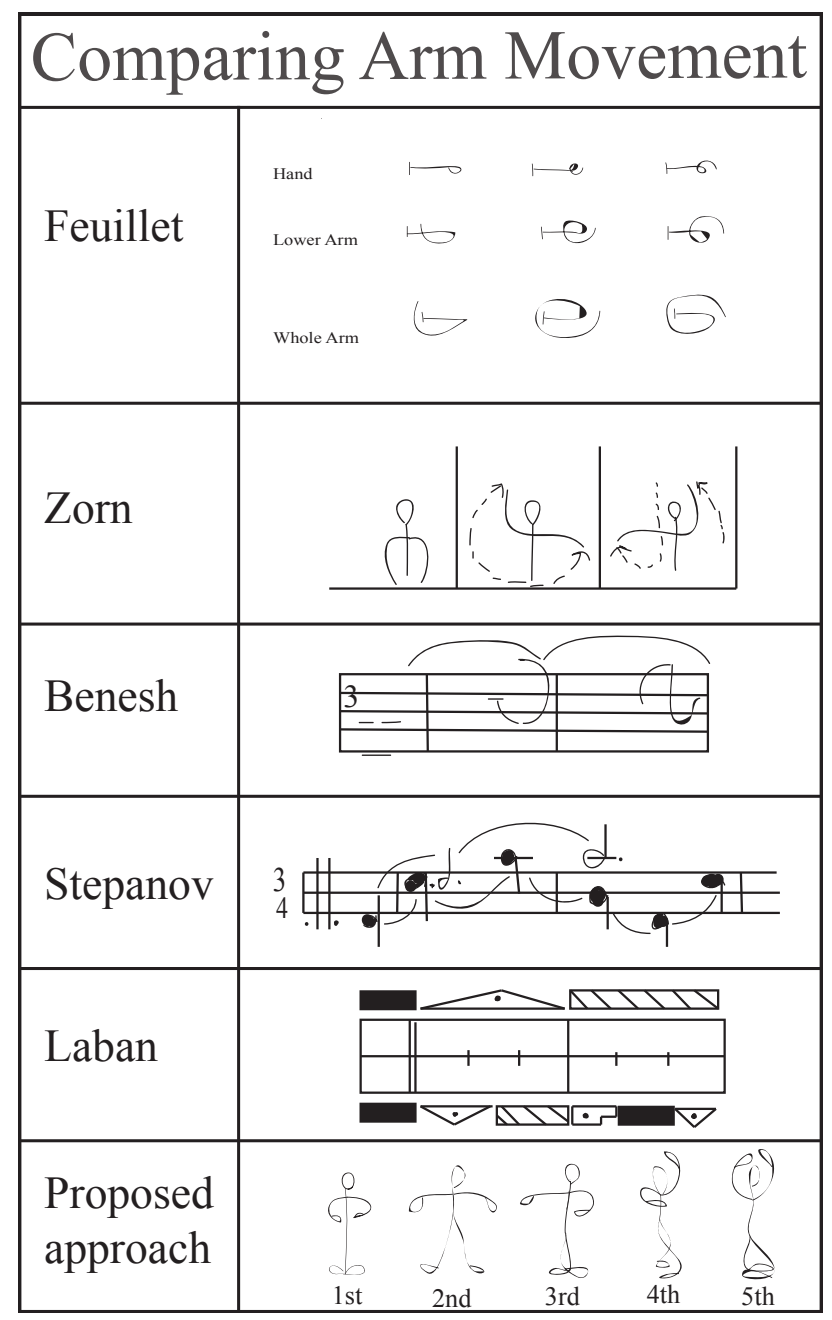

Figure 5.12: Comparing arm position between the common annotation systems

it continues to move forward horizontally, then goes straight down and finally arrives at the side horizontally.

In Labanotation, the first step indicates the arms are in the low position straight down. On count 1 , both arms are moved to the side of the body. The left arm takes 3 counts to arrive to the side position, while the right arm moves continuously sideward and upward over three counts. It moves forward on count 1 of the second measure, downward on count 2, and to the side on count 3. At the same time, the 
left arm moves slowly up over 3 counts.

The notations used for representation of the arm positions in the proposed approach are similar to the Zorn's annotation. The difference is that, they are not using dotted lines to identify in-between movements, instead they show a representative pose to fetch a 3D mini-motion. The movements shown using the dancing avatar provide all of the in-between details.

\subsubsection{Comparison of Jumping Sequence}

Figure 5.13 compares representation of a standard jump sequence using annotations that are commonly used for ballet choreography, with the new annotation.

In Feuillet, a jump sequence starts with putting feet together and bending knees.

A jump into $2^{\text {nd }}$ position follows by bringing the feet together again. A jump while travelling backward places the feet together after landing.

In Zorn's system, the jumping sequence is quite pictorial. The movement indicates the rising into the air and shows the supports as well. The arrowed white pin shows travelling backward.

In Benesh notation, springing into the air is shown by a jump line, a downward curved bow connecting the start and finish of the jump. Jumping backward is shown by adding a black dot to the bow.

In Stepanov system, jump starts with placing feet together in the first position while knees are bent. Two leg gestures show springing into the air. Landing in the second position is shown by supports in the side direction, again with knees bent. This follows by springing again to the first position, then springing backward with feet together when landing. The backward travelling is indicated by backward leg 


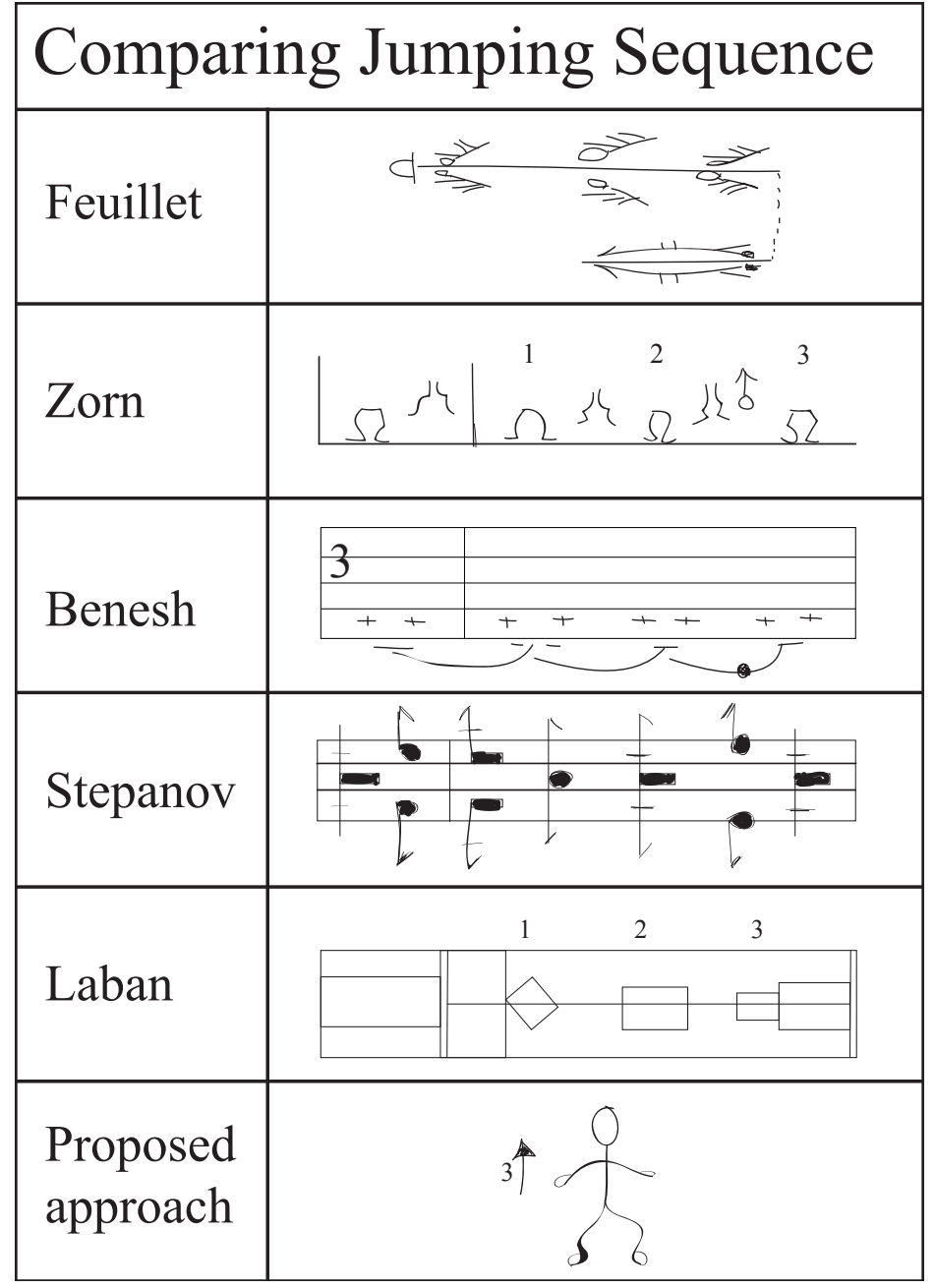

Figure 5.13: Comparing Jumping Sequence between the common annotation systems gestures, even though the leg does not actually go backward while in the air.

In Laban's notation, during the jumping pattern, all supports are in demi-plie. The shape of the symbols show the jump direction. Jumps are indicated by leaving a space between supports, which shows the absence of support. The jumping sequence starts with feet together and knee bent. The space indicates a spring into the air, which is then followed by landing with the feet apart in $2^{\text {nd }}$ position on count 1 . The next jump lands on count 2, feet together. The last jump travels backward landing 
with feet together on count 3 .

The stick-figure shown in the proposed approach, accompanies with a vertical arrow that indicates the jump. Showing the number 3 helps to repeat the corresponding mini-motion three times. Evidently, the ability of retrieving mini-motions helps the proposed approach to greatly simplify the input annotation.

\subsubsection{Comparison of Walking Sequence}

Figure 5.14 compares representation of a walk sequence in 5 well-known annotation systems against the proposed representation.

Feuillet's annotation shows a step forward on the right foot, a step sideward on the left foot, then a step backward on the right foot.

Zorn's annotation of walking sequence is based on these signs: (b) indicates travelling forward (toward the viewer), while (c) shows travelling backward. (d) is a sign for transferring the weight to the right foot, and (e) for transferring the weight to the left foot. In the starting position of (f) weight is on the left foot and the right foot moves forward touching the ground. Forward travelling, soon to take place, is indicated ahead of time. Transferring the weight to the right is followed on count 1 by the position with weight on the right foot. Transferring the weight to the left leg with the right touching to the side on count 2. Next, weight is transferred to the right and indication of travelling backward leads to the potion on count 3 with weight on the right foot.

Benesh uses a curved step line for walking sequence which also indicates the height of the transitory leg. Where the line starts and ends shows which foot is used. Often this is obvious, but after a starting position it needs to be made clear. 


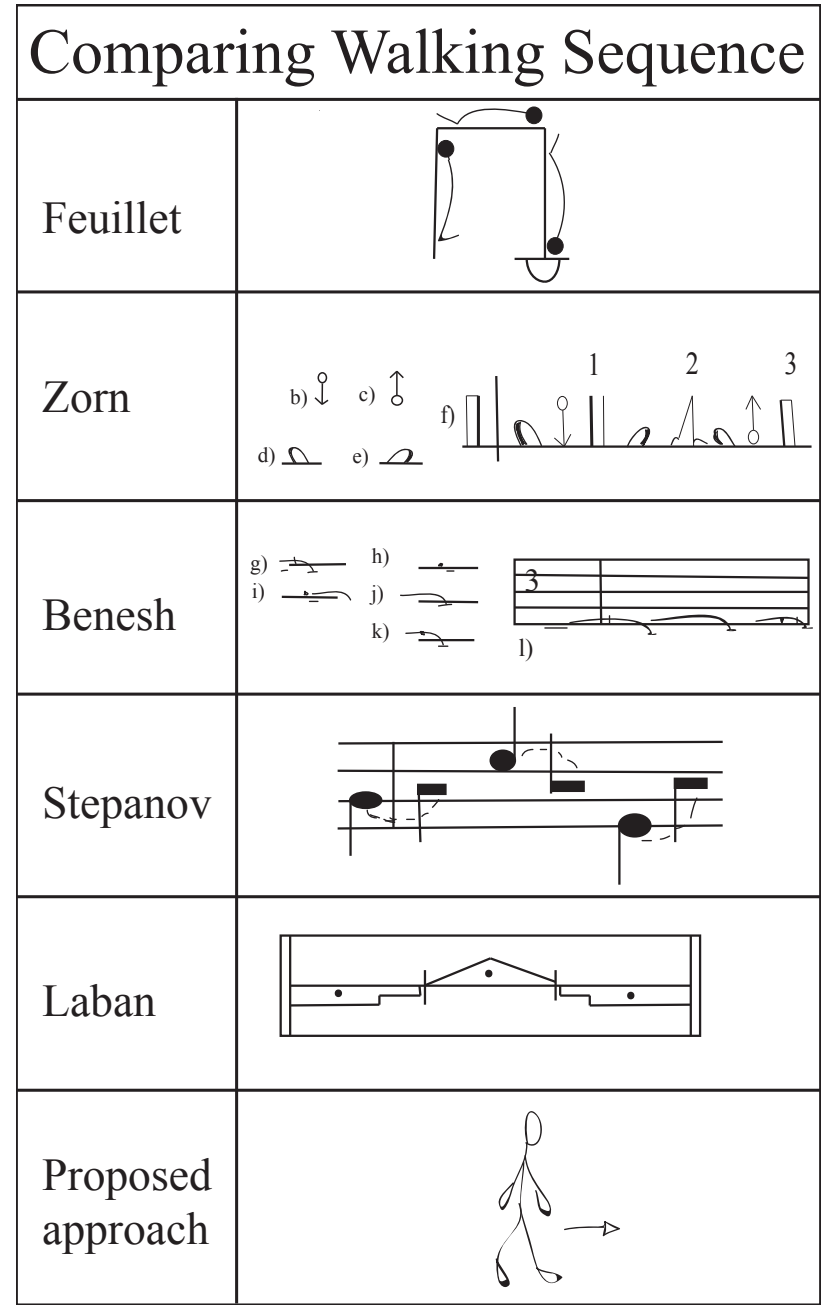

Figure 5.14: Comparing walking sequence between the common annotation systems Therefore, in (g) to show a forward step, the vertical stroke for forward is added to the line. If it is an ending step, the left foot would probably be shown touching the floor as in (h). A step to the left foot is shown by where the line starts as in (i) and ends as in (j). Finally, (k) shows the step backward on the right foot, the backward direction being indicated by the black dot on the step line. An established walking pattern is shown in (l); forward on the right foot, sideward on the left foot and backward on the right. 
Direction of the steps in the Stepanov system is indicated by a preparatory leg gesture tied to the following support sign by dotted bow. As shown in the chart, it starts by a step forward on the right foot, followed by a step to the left on the left foot and then a step backward on the right foot.

In Laban's system the whole body is written in the center column on the staff. Walking is represented by placing the appropriate direction symbol in the support column adjacent to the center line. Placing it on the left, shows steps on the left foot and placing it on the right, shows steps on the right foot. A step is analysed as a transference of weight of the whole body. As shown in the chart, it shows a step forward on the right foot, a step to the left on the left foot and a step backward on the right.

In the proposed annotation, a forward walking sequence can be represented simply by showing a stick-figure and a forward arrow. Other variations of the walk, can be defined by just changing this arrow.

\subsubsection{Comparison of Turning Sequence}

Figure 5.15 compares turning sequence in 5 well-known annotation systems with the proposed representation.

For Feuillet, all of the turnings shown in the chart are turns to the right. For the whole turn, a dot indicates from which side of the symbol the turn sign should be read.

Zorns indicator for turning, provides an element of confusion. His signs use an arrow at the end of the curved or circular lines attached to the base line. Below the turning indication a black pin indicates on which foot the weight is placed. In the 


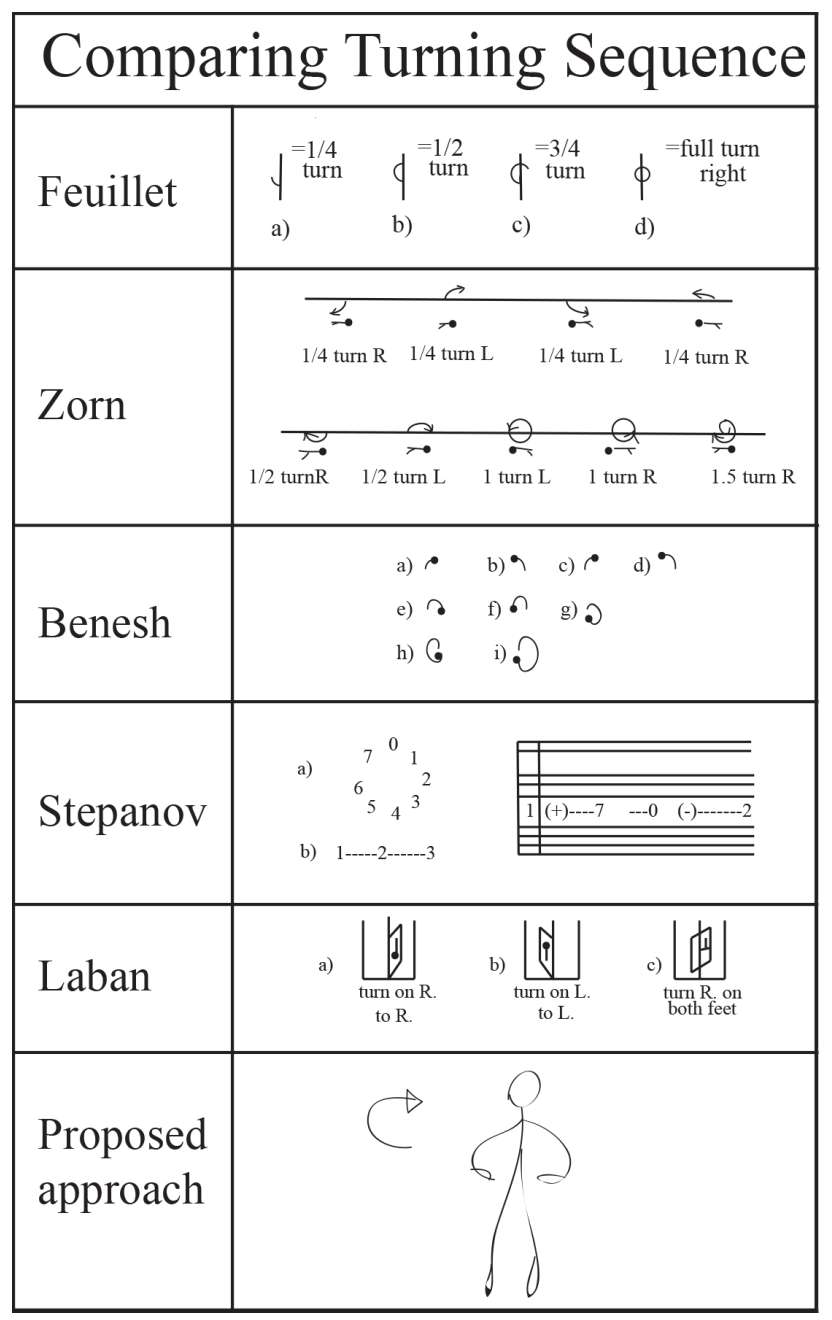

Figure 5.15: Comparing Turning sequence between the common annotation systems first row, it respectively shows a $\frac{1}{4}$ turn to the right on the right foot, a $\frac{1}{4}$ turn to the left on the right foot, a $\frac{1}{4}$ turn to the left on the left foot and a $\frac{1}{4}$ turn to the right on the left foot. The same visual confusion regarding turning direction applies to $\frac{1}{2}$ turns. In the second row, it respectively shows a $\frac{1}{2}$ turn to the right on the right foot, a $\frac{1}{2}$ turn to the left on the right foot, a full turn to the left on the left foot, a full turn to the right on the left foot and one and a half turns to the right on the right foot. 
Benesh notation also uses similar elements for turns. It shows (a) a $\frac{1}{8}$ turn to the right; (b) a $\frac{1}{8}$ turn to the left; (c) a $\frac{1}{4}$ turn to the right and (d) a $\frac{1}{4}$ turn to the left. The $\frac{1}{2}$ turn to the right in (e) forces the performer to end while facing the back of

the room, as does the $\frac{1}{2}$ turn to the left in (f). A full clockwise turn to the right is shown in (g), while (h) is a full anticlockwise turn to the left. The number for the multiple turn is written within the turn circle; (i) shows four turns to the right.

Stepanov annotates the whole body for the turning sequence. Each side of the room has a number for direction, starting with zero for audience and going clockwise. This gives 1 for the right front corner; 2 for the right side, and so on. When a direction is to the right, plus sign is used for turning to the right and a minus sign is used for turning to the left.

For turning sequence, Laban places symbols to the column that is going to turn. Thus, if the symbol is on the right support column, it is going to turn right and vice versa. In the chart, (a) is a full turn to the right on the right foot, (b) is a half turn to the left on the left foot and (c) is a swivel turn on both feet to the right which ends facing front to the audience.

Turning is again simplified in the proposed approach using a stick figure and a curved arrow that can be clockwise or counter-clockwise.

\subsection{Discussion}

The results of the comparisons presented in section 5.3 show that proposed approach can simplify the dance annotation. This is the direct result of using mini-motions which provide additional information than the input annotations. The standard 
annotations are not easy to guess by non-professionals. Using the new annotations not only saves the choreographers' effort, but also plays the role of a visual idea generator. This is initiated from the visual nature of the proposed annotations that can be used in a story-telling process. This intuitive process of dance creation greatly helps choreographers to compose innovative and believable dance patterns.

Figure 5.16 compares the specifications supported in the proposed approach with major related works to this research based on a five point scale (Poor, Fair, Average, Good and Excellent). These specifications are in line with the main objectives described in chapter 1.2 . This chart shows that the proposed approach for sketchbased dance choreography can excellently satisfy the main features of both digital choreography systems and sketch-based systems. The use of menu and keyboard was avoided in the design process to comply with the best practices of sketch-based systems. Also, support of video is an optional feature which was not part of our objectives but can be added in future. 


\begin{tabular}{|c|c|c|c|c|c|c|c|}
\hline & Motion Capture & Sketch Based & Video Based & 3D Environment & 2D Environment & Annotation & Menu and Keyboard Based \\
\hline DanceForms [15] & & & & Poor & Good & Good & Excellent \\
\hline $\begin{array}{l}\text { Web3D Dance } \\
\text { Composer [51] }\end{array}$ & Good & & & Average & & & Fair \\
\hline $\begin{array}{l}\text { Dance Designer } \\
\text { (Choreopro) [17] }\end{array}$ & & & Good & & Fair & & Good \\
\hline Lee et al. [40] & Excellent & Good & & Excellent & & & \\
\hline Motion Master [41] & & Good & & Fair & Average & & \\
\hline James et al. [33] & & Average & Excellent & & Poor & & \\
\hline $\begin{array}{l}\text { Sketch-Based } \\
\text { Dance Choreography }\end{array}$ & Excellent & Excellent & & Excellent & Good & Excellent & \\
\hline
\end{tabular}

Figure 5.16: Comparison of the Sketch-Based Dance Choreography with major related works based on their features. 


\section{Chapter 6}

\section{Conclusions}

In this thesis, a sketch-based approach for creating 3D animations of ballet has been presented. This approach helps dance choreographers intuitively design and animate different dance patterns. It also contributes a stick figure annotation system that converts the timely process of dance annotation to just doodling the desired dance motions. This visual technique for ballet annotation is designed based on a study on traditional ballet annotations and informal interviews with domain experts.

Another contribution of this approach is allowing choreographers preview and edit 3D dance patterns from any angle. To generate realistic 3D motions, a MoCap database has been used. The motions of this database have been pre-processed and skinned using Motion Builder. Then, they are segmented to their mini-motions inside Unity3D. Next, a set of state-machines and blend trees were created using Unity3D's dynamic motion blending mechanism. Finally, using a sketch-based interface the captured mini-motions were smoothly blended while following a trajectory. This user interface is more closely related to the traditional media (pen and paper) used for choreography. The resulting sequence is a moving avatar that can be played as a 3D animation.

The proposed approach is unique in its use of captured motions, an intuitive

method for motion control and its ease of use for non-experts. It helps choreographers dynamically design a dance sequence by progressively retrieving appropriate motions through sketching annotations. This 3D approach for synthesizing dance has an 
added benefit for people interested in printing or publishing the dance steps. The realistic nature of the synthesized movements make it possible to extract the frames of the resulting dance as a sequence of images. This can save a lot of time in drawing and publishing the training materials for dance studios. These printed documents can also help choreographers that still prefer to work on paper.

\subsection{Future Work}

In the future, a formal user study should be conducted to evaluate the usability of this approach for choreography. Multiple directions can be envisioned to conduct the user study. For example, a few choreography tasks can be given to choreographers to perform. Half of these tasks can be designed using standard annotation systems on paper (that can have accompanying videos) and the other half can be created using the proposed approach. Then, the results of both performances can be judged by a ballet master. In this regard, a tablet version of the prototype should be created to emulate the pen and paper metaphor. The decision to use Unity3D for development of the prototype makes it straightforward to publish an app on different platforms.

If the user study shows promising results, creating a rich product for dance choreography is the next step. A crucial part of this product development should be capturing all ballet mini-motions in a MoCap studio. Also, to help choreographers better plan the dance, they should be able to start from a musical score and define the timing of the animations according to that. Integrating and controlling the dance by a musical score is an important direction for future research. Another avenue of ex-

ploration is applying the proposed approach to other applications other than dance. 
The approach presented in this thesis can be customized for other applications such as: fashion shows, figure skating, gymnastics, and marching bands. Finally, this approach can be useful as a 3D story boarding tool for animation studios. 


\section{Bibliography}

[1] Autodesk - motionbuilder. https://www.autodesk.com/MotionBuilder, 2014.

[2] Cmu graphics lab motion capture database. http://mocap.cs.cmu.edu, 2014.

[3] Unity - game engine. https://unity3d.com, 2014.

[4] J. Adshead-Lansdale and J. Layson. Dance History: An Introduction. Routledge, 1994.

[5] J. Anderson. Ballet \& modern dance: a concise history. Princeton Book Co., 1992.

[6] Lisa Anthony and Jacob O. Wobbrock. A lightweight multistroke recognizer for user interface prototypes. In Proceedings of Graphics Interface 2010, GI '10, pages 245-252, Toronto, Ont., Canada, Canada, 2010. Canadian Information Processing Society.

[7] T. Arbeau and L. Fonta. Orchésographie. Bibliotheca musica Bononiensis. Forni, 1888.

[8] S. Au. Ballet and Modern Dance. World of Art. Thames \& Hudson, 2002.

[9] S. Au. Ballet and Modern Dance. World of Art. Thames \& Hudson, 2002.

[10] R. Benesh. Benesh Movement Notation. Institute of Choreology, 1955.

[11] A. Bland. A history of ballet and dance in the western world. Praeger, 1976. 
[12] L.A. Blom and L.T. Chaplin. The Intimate Act of Choreography. Pitt paperback. University of Pittsburgh Press, 1982.

[13] Armin Bruderlin and Lance Williams. Motion signal processing. In Proceedings of the 22Nd Annual Conference on Computer Graphics and Interactive Techniques, SIGGRAPH '95, pages 97-104, New York, NY, USA, 1995. ACM.

[14] A Dancer's Days Blog by Rhiannon Pelletier. The secret to turning. http://adancersdays.blogspot.fr/2011/03/secret-to-turning.html, 2014.

[15] Tom Calvert, Lars Wilke, Rhonda Ryman, and Ilene Fox. Applications of computers to dance. IEEE Comput. Graph. Appl., 25(2):6-12, March 2005.

[16] F. Caroso, J. Sutton, and F.M. Walker. Courtly Dance of the Renaissance: A New Translation and Edition of the Nobiltà Di Dame (1600). Dover Books on Music Series. Dover Publications, 1995.

[17] ChoreoPro. Dance designer. https://www.choreopro.com, 2014.

[18] M. Clarke and C. Crisp. The history of dance. Crown Publishers, 1981.

[19] James Davis, Maneesh Agrawala, Erika Chuang, Zoran Popović, and David Salesin. A sketching interface for articulated figure animation. In Proceedings of the 2003 ACM SIGGRAPH/Eurographics Symposium on Computer Animation, SCA '03, pages 320-328, Aire-la-Ville, Switzerland, Switzerland, 2003. Eurographics Association.

[20] Edilson de Aguiar, Carsten Stoll, Christian Theobalt, Naveed Ahmed, HansPeter Seidel, and Sebastian Thrun. Performance capture from sparse multi-view 
video. 2008.

[21] J. Dewald. Europe 1450 to 1789: encyclopedia of the early modern world. Number v. 6 in Europe 1450 to 1789: Encyclopedia of the Early Modern World. Charles Scribner's Sons, 2004.

[22] N. Eshkol and A. Wachmann. Movement Notation. Weidenfeld and Nicolson, 1958.

[23] R.A. Feuillet. Chorégraphie Ou L'art de Décrire la Dance. Monuments of music and music literature in facsimile. Ser. 2. Broude Brothers, 1700.

[24] Pablo Figueroa, J. Arcos, D. Rodriguez, J. Moreno, and Faramarz Samavati. A pen and paper interface for animation creation. In GRAPP, pages 364-371, 2014.

[25] Margaret S. Geroch. Motion capture for the rest of us. J. Comput. Sci. Coll., 19(3):157-164, January 2004.

[26] Michael Gleicher and Peter Litwinowicz. Constraint-based motion adaptation. pages 65-94, 1998.

[27] A.H. Guest. Dance notation: the process of recording movement on paper. Dance Books, 1984.

[28] A.H. Guest. Dance notation: the process of recording movement on paper. Dance Books, 1984.

[29] A.H. Guest. Choreo-graphics: A Comparison of Dance Notation Systems from the Fifteenth Century to the Present. Gordon and Breach, 1998. 
[30] Ann Hutchinson Guest. Dance notation. Perspecta, 26:pp. 203-214, 1990.

[31] A.L. Haskell. Ballet: A Complete Guide to Appreciation : History, Aesthics, Ballets, Dancers. Pelican books, A122. Penguin Books, 1955.

[32] J. Homans. Apollo's Angels: A History of Ballet. Random House Publishing Group, 2010.

[33] Stuart James, Manuel J. Fonseca, and John P. Collomosse. Reenact: Sketch based choreographic design from archival dance footage. In ICMR, page 313, 2014.

[34] Joaquim Jorge and Faramarz Samavati, editors. Sketch-based Interfaces and Modeling. Springer London, 2011.

[35] L. Kirstein, M. Stuart, G. Balanchine, and C. Dyer. The Classic Ballet: Basic Technique and Terminology. Borzoi book. Knopf Doubleday Publishing Group, 2004.

[36] F. Kleiner. Gardner's Art through the Ages: A Global History. Gardner's Art Through the Ages. Cengage Learning, 2008.

[37] Lucas Kovar, Michael Gleicher, and Frederic H. Pighin. Motion graphs. pages 473-482, 2002.

[38] Harold W. Kuhn. The hungarian method for the assignment problem. Naval Research Logistics Quarterly, 2:83-97, 1955.

[39] R. Laban. Principles of Dance and Movement Notation: With Basic Movement Graphs and Their Explanation. The Dance Horizons series. 20. 1970. 
[40] Jehee Lee, Jinxiang Chai, Paul S. A. Reitsma, Jessica K. Hodgins, and Nancy S. Pollard. Interactive control of avatars animated with human motion data. $A C M$ Trans. Graph., 21(3):491-500, July 2002.

[41] Q. L. Li, W. D. Geng, T. Yu, X. J. Shen, N. Lau, and G. Yu. Motionmaster: Authoring and choreographing kung-fu motions by sketch drawings. In Proceedings of the 2006 ACM SIGGRAPH/Eurographics Symposium on Computer Animation, SCA '06, pages 233-241, Aire-la-Ville, Switzerland, Switzerland, 2006. Eurographics Association.

[42] Yang Li. Protractor: A fast and accurate gesture recognizer. In Proceedings of the SIGCHI Conference on Human Factors in Computing Systems, CHI '10, pages 2169-2172, New York, NY, USA, 2010. ACM.

[43] Thomas B. Moeslund, Adrian Hilton, and Volker Krger. A survey of advances in vision-based human motion capture and analysis. pages 90-126, 2006.

[44] Elahe R. Moghaddam, Javad Sadeghi, and Faramarz Samavati. Sketch-based dance choreography. In International Conference on Cyberworlds (CW) 2014. IEEE Computer Society, Oct 6-8 2014.

[45] Benesh movement notation. Royal academy of dance. https://www.rad.org.uk, 2014.

[46] D.A. Norman and S.W. Draper. User centered system design: new perspectives on human-computer interaction. New Perspectives on Human-Computer Interaction Series. Lawrence Erlbaum Associates, 1986. 
[47] Art of Ballet. http://www.artofballet.com, 2010.

[48] Luke Olsen, Faramarz F. Samavati, Mario Costa Sousa, and Joaquim A. Jorge. Sketch-based modeling: A survey. Computers \& Graphics, 33(1):85-103, Feb 2009.

[49] Masaki Oshita. Pen-to-mime: A pen-based interface for interactive control of a human figure. In Proceedings of the First Eurographics Conference on SketchBased Interfaces and Modeling, SBM'04, pages 43-52, Aire-la-Ville, Switzerland, Switzerland, 2004. Eurographics Association.

[50] R. Rastall. L'art et instruction de bien dancer (Michel Toulouze, Paris). S. R. Publishers, 1971.

[51] A. Saint-Léon and R. Lister. Stenochoreography, Or, The Art of Writing Dancing Swiftly. Raymond Lister, 1992.

[52] Asako Soga, Bin Umino, Takami Yasuda, and Shigeki Yokoi. A system for choreographic simulation of ballet using a $3 \mathrm{~d}$ motion archive on the web. In AMDO, pages 227-238, 2004.

[53] V.I. Stepanov. Alphabet of movements of the human body: a study in recording the movements of the human body by means of musical signs. Dance Horizons, 1969.

[54] B. Taper. Balanchine, a Biography. University of California Press, 1996.

[55] American Ballet Theatre. Ballet dictionary. http://www.abt.org/education/dictionary/, 2014. 
[56] Matthew Thorne, David Burke, and Michiel van de Panne. Motion doodles: An interface for sketching character motion. ACM Trans. Graph., 23(3):424-431, August 2004.

[57] Radu-Daniel Vatavu, Lisa Anthony, and Jacob O. Wobbrock. Gestures as point clouds: A \$p recognizer for user interface prototypes. In Proceedings of the 14 th ACM International Conference on Multimodal Interaction, ICMI '12, pages 273280, New York, NY, USA, 2012. ACM.

[58] K. Vatsyayan. Bharata The Natyasastra. Sahitya Akademi, 2006.

[59] D.G. Wilkins, B. Schultz, and K.M. Linduff. Art Past, Art Present (with MyArtKit Student Access Code Card). Prentice Hall PTR, 2009.

[60] A. Williamson. Contemporary Ballet. Rockliff, 1946.

[61] Jacob O. Wobbrock, Andrew D. Wilson, and Yang Li. Gestures without libraries, toolkits or training: A $\$ 1$ recognizer for user interface prototypes. In Proceedings of the 20th Annual ACM Symposium on User Interface Software and Technology, UIST '07, pages 159-168, New York, NY, USA, 2007. ACM.

[62] F.A. Zorn, A.J. Sheafe, and B.P. Coates. Grammar of the Art of Dancing, Theoretical and Practical: Lessons in the Arts of Dancing and Dance Writing (choreography). Heintzemann Press, 1905. 